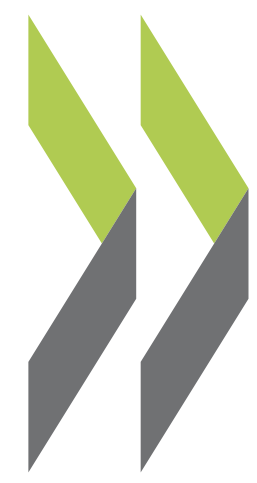

OECD Economics Department Working Papers No. 29

\title{
Method of Calculating
} Effective Exchange Rates and Indicators of Competitiveness 


\section{OECD \\ DEPARTMENT \\ OF ECONOMICS AND STATISTICS}

\section{WORKING PAPERS}

N. 29: METHOD OF CALCULATING EFFECTIVE EXCHANGE

RATES AND INDICATORS OF COMPETITIVENESS

by

Martine Durand

General Economics Branch

Balance of Payments Division

February 1986

OCEDE

需恶

(0ECD 



\title{
OECD \\ DEPARTMENT \\ OF ECONOMICS AND STATISTICS
}

\section{WORKING PAPERS}

No. 29: METHOD OF CALCULATING EFFECTIVE EXCHANGE RATES AND INDICATORS OF COMPETITIVENESS

\author{
by \\ Martine Durand \\ General Economics Branch \\ Balance of Payments Division
}

February 1986

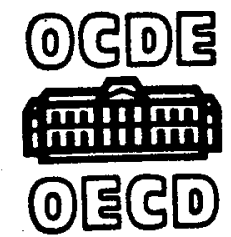





\section{ECONOMICS AND STATISTICS DEPARTMENT}

\section{WORKING PAPERS}

This series of Working Papers is designed to make available, to a wider readership, selected studies which the Department has prepared for use within OECD. Authorship is generally collective, but main individual authors are named. The Papers are generally available in their original language, English or French, with a summary in the other.

Comment on the Papers is invited, and may be sent to OECD, Department of Economics and Statistics, 2 rue André Pascal, 75775 Paris Cedex 16, France. Additional copies of the Papers on a limited basis can be forwarded on request.

ORGANISATION FOR ECONOMIC CO-OPERATION AND DEVELOPIENT

Copyright OECD 1985

W. $6167 \mathrm{E}$

34533 

METHOD OF CALCULATING EFFECTIVE EXCHANGE RATES AND INDICATORS OF COMPETITIVENESS

by

Martine Durand

General Economics Branch

Balance of Payments Division

February 1986

I gratefully acknowledge the comments received from Mr. Feiner and Mr. Herd. 

This paper describes the method of calculation of competitiveness indicators and effective exchange rates that are published half-yearly by the Secretariat in the Economic Outlook. This calculation is based on a double-weighting principle: the procedure calculates the relative importance of different countries on each market according to the relative importance of these markets for the country in question. The calculations include the impact of the domestic producer in each market, i.e. a particular country is considered as a competitor to other exporters to its own market. On the other hand, in calculating the importance of each competitor to a given country the share of the country in question is excluded in every market, i.e. a country cannot compete with itself. Weighting matrices have been constructed for each year, starting in 1970, and have been used to define and compute relative indices of costs, prices and exchange rates.

\section{*ネ*ネネネネネネネ}

Le présent article décrit la méthode de calcul des indicateurs de compétitivité et de taux de change effectifs que le secrétariat publie régulièrement dans les Perspectives Economiques. Le calcul est fondé sur un principe de double pondération : on cherche à mesurer l'importance relative de différents pays sur différents marchés en fonction de 1'importance relative de ces divers marchés pour les pays en question. Les calculs prennent en compte 1'impact des marchés domestiques, à savoir qu'un pays est lui-même un coricurrent sur son propre marché. Par contre, la mesure de 1'importance relative des concurrents d'un pays sur chaque marché exclut le pays en question, ce dernier ne pouvant être en concurrence avec Iui-même. Des matrices de poids ont été construites depuis 1970 et ont servi à la definition et au calcul d'indices relatifs de coût et de prix et de taux de change. 



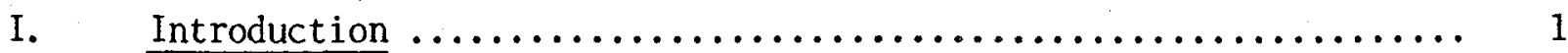

II. The weighting method adopted $\ldots \ldots \ldots \ldots \ldots \ldots \ldots \ldots \ldots \ldots \ldots \ldots \ldots \ldots \ldots$

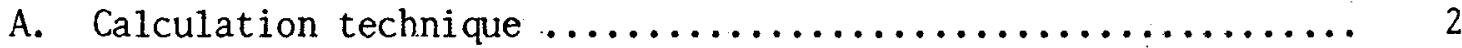

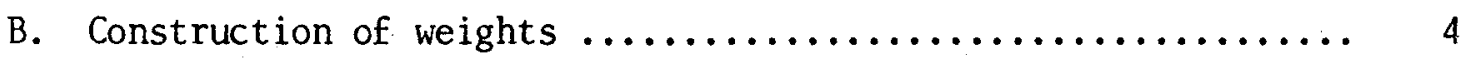
C. Matrices obtained ................................ 6

III. Relative indicators of competitiveness and effective

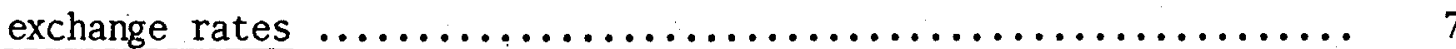

A. Method of index calculation $\ldots \ldots \ldots \ldots \ldots \ldots \ldots \ldots \ldots \ldots \ldots$

B. Choice of relative indices of competitiveness ............. 9

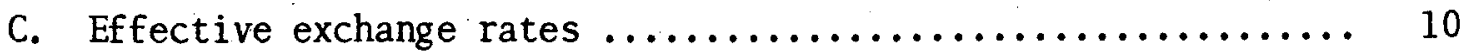

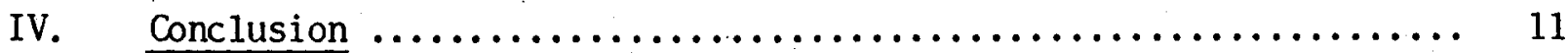

Annexes:

1. Gross production of manufactured products $\ldots \ldots \ldots \ldots \ldots \ldots \ldots \ldots$

2. Matrices of weights for the calculation of effective exchange rates and competitiveness indicators $\ldots \ldots \ldots \ldots \ldots . \ldots . \ldots 15$ 
TABLE OF CONTENTS (cont'd)

Page

3. Trend of weighting matrices over time $\ldots \ldots \ldots \ldots \ldots \ldots \ldots \ldots \ldots$

4. Quarterly values of indices of relative competitiveness and effective exchange rates $\ldots \ldots \ldots \ldots \ldots \ldots \ldots \ldots \ldots \ldots \ldots$ 


\section{Ir.troduction}

1. One of the difficulties commonly encountered when trying to determine the competitive position of a given country's economy vis-à-vis those of its trading partners is that of comparing in absolute terms the indicators selected as being representative of a measure of competition. These indicators as a rule relate to prices and costs, and data on levels are rarely available or comparable for all the relevant sectors for a sufficient number of ccuntries. This means that, as a rule, it is not possible to ascertain, for example, whether unit costs in a given country's industry are higher or lower than those in its partners' industries. The only data available to us that are homogeneous in respect of a large number of countries are in fact indices of costs and prices. These obviousiy cannot provide any information about absolute cost and price levels. However, they do provide a comparison in relation to a base year of the movement of costs and prices in different countries.

2. While countries' individual cost and price movements may be of interest, it is sometimes more appropriate to measure the trend of their relative competitive position. If, for example, costs and prices in a particular country have risen steeply, it will be useful to compare the situation with that in a number of its trading partners so as to ascertain whether it is part of a general trend, in which case there is no effective loss of competitiveness, or whether, on the contrary, the competitive position of a particular country has deteriorated by comparison with that of other countries. To do this, we define relative indicators of competitiveness that compare a given country's prices or costs with an arithmetic or geometric weighted average of prices or costs in a number of partner countries.

3. We are seeking to measure what is in fact overall trade competitiveness; in other words, we are trying to find out how to gauge the relative performance of a number of countries on a number of markets. This being so, we shall define a weighting system appropriate to calculating various measures of relative competitiveness. (chiefly effective exchange rates, relative unit labour costs and relative unit export values). Several definitions of a weighting system are possible. For example, weightings can be based on bilateral trade. Such a system in which each weight is defined 
for each country $i$ as the ratio of country i's exports (or imports or trade) with respect to country $j$ to its total exports (or imports or trade) has the advantage of simplicity. On the other hand, it does not allow for the effects induced by third-country markets since it is based on bilateral trade alone. Specifically, it does not lend itself to measuring the competition the two countries engage in on a third market.

4. These considerations are taken account of in the weighting scheme used by the OECD Secretariat to construct effective exchange rates and indicators of competitiveness. This note describes the derivation of this scheme in detail and presents the resulting measures of competitive position.

\section{The weighting miethod adopted}

5. In order to be able to take account both of bilateral competition and of induced third-market competition, it is necessary to use a system of double weighting which distinguishes the role played by each country as both supplier and market. The method must indeed take account of the relative importance of all competitcrs on each market, and of the importance of each market, including the domestic market, for each supplier. Using the double weighting method, we construct, for each of the countries considered to be suppliers, the same number of indicators of competition as it has markets for its products, and we then amalgamate them into a composite indicator according to the importance each market represents for the country considered.

6. In addition, in constructing a weighting system to be used to construct a measure of the relative competition of a number of countries on joint markets, account has to be taken of products actually competing on the markets and which therefore can be traded. It is of course difficult to have a precise idea of which of a country's goods are tradeable and this is why we considered that, generally speaking, manufacturing industry was a satisfactory approximation of a tradeable goods sector.

\section{A. Calculation technique}

7. Let us consider $n$ exporting countries and m markets, with $x_{i j}$ supplier i's exports of manufactures to market $j$. The matrix of order $(n, m)$ 
constructed in this way will have zero diagonal elements. In order to take proper account of the pattern of competition on each market, the domestic producer must be included in the calculations. Hence elements $X_{i i}$ in the matrix are defined as being equal to domestic production of manufactures, less exports. The basic matrix is therefore of the following form:

markets

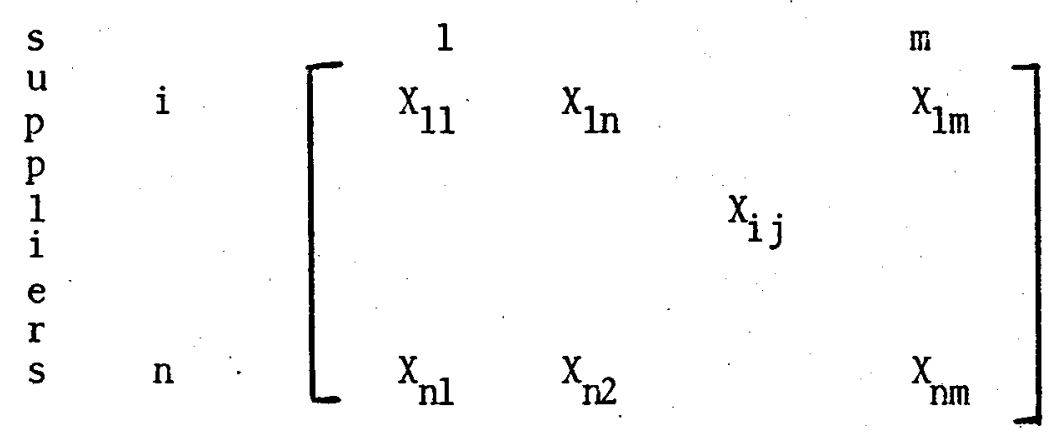

For each country $\bar{i}$ we can define a vector of crder $n-1$ which measures the relative importance of its competitors on market $\bar{j}$, i.e. $z^{\frac{i}{j}}$ such that

$$
\left.z \frac{\bar{j}}{\sum_{\substack{i=1 \\ i \neq 1}}^{n} x_{i \bar{j}}}\right)\left(\frac{x_{i \bar{j}}}{\substack{i=1 \\ i \neq 1} \ldots n}\right.
$$

which gives the ratio of each competitor i's exports to country $\bar{j}$ to total. exports to country $\bar{j}$ from country $\bar{i}$ 's $n-1$ competitors. (1)

8. The vector $2 \bar{j}$ measures the importance of domestic producticn on market $\bar{j}$ via the ratio of country $\bar{j}$ 's production to the total supply of manufactures, excluding those from country $\vec{i}$, on market $\vec{j}$. If now, for each

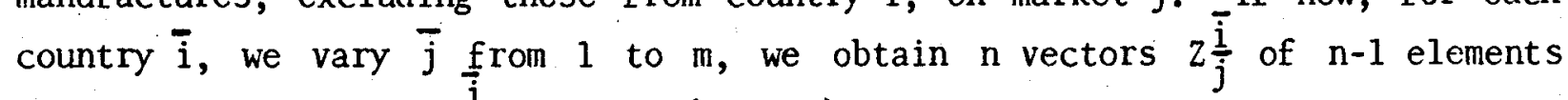
which define a matrix $z^{\bar{i}}$ of order $(n-1, m)$ :

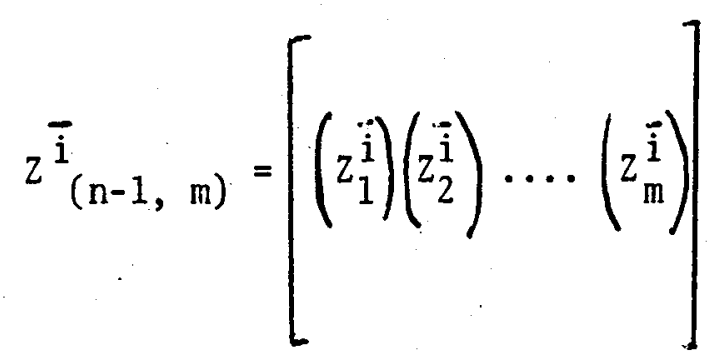


We obtain similar matrices for the $n$ countries. These $n$ matrices $z^{1}, \cdots-z^{n}$ of order $\left(n-1, m_{i}\right)$ give for each country the state of competition on each of its markets.

9. In a second stage we attempt to measure the relative importance of each of the m markets for the $n$ countries considered. This may be measured in the following way: let $\overline{\mathrm{i}}$ be the country considered; we define the vector $\mathrm{Y}^{\overrightarrow{\mathrm{i}}}$ :

$$
Y^{\bar{i}}=\left(\frac{X \bar{i} j}{\sum_{j=1}^{m} X-\overline{i j}}\right) \quad j=1, \ldots m
$$

of order $(\mathrm{m}, 1)$ which represents the ratio of the value of country $\bar{i}$ 's exports to each of its markets $j(i \neq j)$ or of the value of production sold on the domestic market $(i=j)$ to the total value of manufactures produced by $\bar{i}$.

10. We can now define each country i's overall export competitiveness in terms of the vector $W^{\bar{i}}$ which is equal to the product of the matrix $z^{\dot{i}}$ and the vector $\mathrm{Y}^{\hat{\mathrm{i}}}$, i.e.:

$$
\mathrm{w}^{\overline{\mathrm{i}}}(\mathrm{n}-\mathrm{i}, 1)=\mathrm{z}^{\ddot{\mathrm{i}}}(\mathrm{n}-\mathrm{i}, \mathrm{m}) * \mathrm{Y}^{\ddot{\mathrm{i}}}(\mathrm{m}, 1)
$$

We thus obtain $n$ vectors $w^{\vec{i}}$ of order $(n-1)$ and with generic elements

$$
\left(w_{k}^{\bar{i}}\right)_{\substack{k=1 \\ k \neq \bar{i}}, \ldots, n}
$$

which makes it possible, if we set $w \vec{i} \frac{\vec{i}}{i}=0$, to obtain the matrix $w$ of weights of order $(n, n)$ that we needed.

\section{B. Construction of weights}

11. Weighting matrices were constructed in accordance with the method described in Section A for the years 1970 to 1984 . Using the same notation; we considered that $n=23$ or $n=15$ countries supplied $m=29$ markets. The matrices used to construct effective exchange rates and indicators of relative competitiveness are of different orders, $23 \times 29$ in the first case and $15 \times 29$ in second, because of differences in data availability as between nominal exchiange rate indices and cost and price indices. In calculating effective 
exchange rates we took into account the 23 OECD Member countries, while for the relative price or cost indices only a sub-group of 15 countries (United States, Canada, Japan, Germany, France, United Kingdom, Italy, Belgium, Netherlands, Austria, Finland, Sweden, Norway, Denmark and Switzerland) was included in the calculation. Where markets are concerned, we considered the 23 countries of the OECD area and the six non-OECD areas (the high and low absorptive capacity OPEC countries, the other oil-producing developing countries, the newly industrializing countries, the middle- and low-income countries and the East European countries, including the USSR).

12. The data needed for constructing the basic $X$ matrices comprise manufactures exports and output. In order to reconcile the different sources, particularly those for foreign trade and those for output, the ISIC (International Standard Industrial Classification) was adopted. The foreign trade data sources do not present any major problems, which is far from true of output data sources, in respect of which various approximations and interpolations were necessary. Broadly speaking, the data were obtained in the following way:

-- The annual values (expressed in dollars) of each supplier country's manufactures exports to each of its markets (the $x_{i j}, i \neq j$ ) derive from the OECD's Series C foreign trade file and cover the period 1970-1984.

-- The data on output of manufactures (needed to construct the $x_{i i}$ ) derive from various sources depending on the country:

- For eleven countries, USA, Canada, Germany, France, United Kingdom, Japan, Italy, Australia, Belgium, Netherlands and Sweden, the figures were taken from the OECD's COMTAP database for the period 19.70-1980. For the subsequent years the output data were extrapolated as follows: gross output of manufactures was regressed on valued added in manufacturing industry over the 11-year period (1970-1980) and the coefficients obtained were used to complete the output series using data on value added in industry derived from the OECD national accounts statistics. 
- For eight countries, Finland, Greece, Austria, Denmark, Norway, Portugal, Spain and Turkey, input/output matrices deriving from the United Nations and available for the years 1970 and 1975 were used. For these two years we calculated gross output and value added in the sectors comprised by manufacturing industry and then their ratio for 1970 and 1975. Taking the average of this ratio, we were able to interpolate gross output figures for the missing years using the data on value added in manufacturing derived from the OECD national accounts statistics.

- For Ireland, Iceland and New Zealand, we cbtained series for value added in manufacturing. Taking the average of the ratio calculated for the eight preceding countries (ratio of 1970 and 1975) from UN data, we constructed a series of gross output of manufactures.

- For Switzerland, a survey published in 1980 by the office Fédéral de la Statistique provides 1975 data for value added and gross output in manufacturing. On the other hand, there are no historical data for value added in industry. The ratio of value added in industry to total value added was calculated for 1975 . This was used to interpolate the missing series of value added in industry from GDP figures. The ratio of value added in industry to gross output in 1975 was then used to interpolate the output series required.

\section{The matrices obtained}

13. Some of the basic data used to construct the matrices $X$ are set out in Arinex 1. From these data we calculated weighting matrices in accordance with the nethod described in Section A. These matrices, denoted $W_{T}, T=1970$, ,-- 1984 , are also given in Annex 2. They are made up of two sets, the set of matrices of order $23 \times 23$ used for effective exchange rates and the set of niatrices of order $15 \times 15$ needed to construct indicators of competitiveness. 
14. Analysis of the trend over time of the weights obtained shows that they are very stable for most countries. However, there are some divergences, as the tables in Annex 3 show. In particular, vis-à-vis the USA, Canada has a weight that tends to decline, whereas that of Japan tends to increase. Furthermore, vis-à-vis Germany, the USA weighting declines over the period, whereas that of the United Kingdom increases and the share of the latter decreases fairly steeply vis-à-vis Canada, mainly to the benefit of Japan.

III. Relative indicators of competitiveness and effective exchange rates

\section{A. Method of index calculation}

15. The weighting system calculated above was used to define relative indices. Two distinct problems had to be solved: first, that of defining the "relativity" of the index of a variable and, second, that of incorporating weights that are variable over time in that definition.

16. With any fixed weighting system, relative indicators can be defined in two ways:

-- either by comparing an index for one country with the weighted average of the same index for the partner countries;

-- or by comparing the rate of growth of the index with the weighted average of the growth rates between two periods of the indices of the other courtries, and then applying the result obtained to the value of the relative index for the previous period.

17. Moreover, one may choose between a geometric and an arithmetic weighted average. With an arithmetic average, both methods of calculating relative indices only yield more or less identical results if the gross indices vary in similar proportions over time. This drawback disappears if a geometric weighted average is used. Thus, according to the first method, we define

$$
\text { IREL }_{i, t}=I_{i, t} / \prod_{\substack{j=1 \\ j \neq i}}^{n} I_{j, t}^{\alpha_{j}}
$$


or if

$$
\begin{array}{r}
M_{t}=\prod_{\substack{j=1 \\
j \neq i}}^{n} I_{j, t} \alpha_{j}^{i} \\
\text { IREL }_{i, t}=I_{i, t^{\prime}} M_{t}
\end{array}
$$

According to the second we define

$$
\begin{aligned}
& \text { IREL }_{i, t}^{\prime} \quad=\quad I_{i, t} /\left[\prod_{\substack{j=1 \\
j \neq i}}^{n}\left(\frac{I_{j, t}}{I_{j, t-1}}\right)^{\alpha_{j}^{i}} \times M^{\prime} t-1\right] \\
& \text { where } \quad M_{o}^{\prime}=\prod_{\substack{j=1 \\
j \neq i}}^{n}\left(I_{j, 0}\right)^{\alpha_{j}^{i}} \\
& \text { and } M_{t}^{\prime}=\prod_{\substack{j=1 \\
j \neq i}}^{n}\left[\left(\frac{I_{j, t}}{I_{j, t-1}}\right)^{\alpha}{ }_{j}^{i} * M^{\prime} t-1\right] \text { for all } t>0
\end{aligned}
$$

Hence

$$
\operatorname{IREL}_{i, t}^{\prime}=I_{i, t} / \dot{M}_{t}^{\prime}
$$

which can be written

$$
\operatorname{IREL}_{i, t}^{\prime}=I_{i, t} /\left[\prod_{\substack{j=1 \\ j \neq i}}^{n}\left(I_{j, t}^{\alpha_{j}^{i}}\right) *\left(\frac{M^{\prime} t-1}{\prod_{\substack{j=1 \\ j=i}}^{n} I_{j, t-1}} \alpha_{j}^{i}\right)\right]
$$

$$
\text { or else } \text { IREL' }_{i, t}=I_{i, t} /\left(M_{t} * \frac{M^{\prime} t-1}{M t-1}\right)
$$

We know that, for $t=0$

$$
M=M_{0}^{\prime}=\prod_{\substack{j=1 \\ j \neq i}}^{n}\left(I_{j, 0}\right)^{\alpha_{i}^{j}}
$$

Assuming :

$$
M_{t-1}=M_{t-1}^{\prime} \text { for all } t>0
$$

then

$$
M^{\prime}{ }_{t}=M_{t} *\left(\frac{M^{\prime} t-1}{M t-1}\right)=M_{t}
$$


which implies that $M_{t}=M^{\prime}{ }_{t}$ for all $t$ and therefore $I_{R E L}{ }_{i, t}=I^{\prime} L^{\prime}{ }_{i, t}$ for all $\mathrm{t}$.

We therefore decided to use the first method, defining a relative index as the comparison of the index of one country with the geometric weighted average of the same index for the other countries.

18. The second problem concerns the incorporation of weights that are variable over time. Here again, there are two possibilities. The first is to define the relative indices in such a way that changes therein only reflect changes in the current indices. This method implies that the level of a composite index does not change with a change in the weighting system. The second method, the one we chose, implies on the contrary that, ceteris paribus, a change in the weights does result in a change in the value of the relative index. In other words, the first method amounts to resetting the index at the beginning of each year on the basis of the previous year's weights, whereas the second does not. The method for calculating a relative index is therefore the following:

$$
\begin{aligned}
& \forall=1, \ldots n \\
& \ln \text { IREL }_{i, t}=\ln I_{i, t}-\sum_{\substack{j=1 \\
j \neq i}}^{n} \alpha_{j, t-1}^{i} * \operatorname{lnI}{ }_{j, t}
\end{aligned}
$$

\section{B. Choice of relative indices of competitiveness}

19. To compare countries' performances we use cost and price indices. Because of the problem of cross-country consistency of data, it is customary to use indices of unit labour costs in industry and of unit values of manufacturing exports. Labour costs do of course represent a part of costs, but it is very difficult to collect reliable and homogeneous data for a sufficiently large number of countries. 
20. Furthermore, where most countries are concerned, export prices are approximated by a unit export value index. This has an advantage in that the data relate to goods that have been recorded by the customs authorities as having left the national territory and that they are representative of products that are actually competing on foreign markets. On the other hand, this index excludes "exportable" goods, which may be a disadvantage since there is a danger that possible losses of competitiveness will not be taken into account, whereby potentially exportable goods were not in fact exported because they were too highly priced.

21. On the whole, labour cost indicators are a better measure of competitiveness than export price indicators. This is because movements in the latter may reflect changes in exporters' profit margins. It would therefore be preferable to base analyses either on labour costs alone or on labour costs and export prices, linking them up for example with changes in flows of traded goods and in market share gains or losses. The indices calculated here thus show trends (by comparison with the base year 1970) in the relative indicators of unit labour costs and of export prices over the long run in order to allow for the lag structure in the relationship linking trade volume and competitiveness.

22. We could also compare the producer prices for manufactures (and not consumer prices which cover a set of goods that are not necessarily exposed to foreign competition); this last indicator may be considered as a compromise between the two previous ones since it reflects all costs but also includes mark-up behaviour (2). Annex 4 contains the series of relative labour costs and relative export prices that the Secretariat publishes regularly in Economic Outlook.

\section{Effective exchange rates}

23. The weighting matrices calculated earlier were also used to calculate effective exchange rates since changes in exchange rates are also an indicator of gains or losses of competitiveness. The method for calculating the effective exchange rate is that given in paragraph 10. However, it is important to specify the definition of the exchange rate index since in this case (by contrast with the indicators of competitiveness based on costs and 
prices) level data for exchange rate values do exist. The purpose of the effective exchange rate is to measure changes in relative currency parities over the period of floating exchange rates. Here, the base period chosen was the 1st quarter 1970, which was the last period in which all currencies involved were'fixed. For each country we defined an exchange rate index by calculating the ratio of the average exchange rate observed during the first quarter of 1970 of the currency of the country considered against the dollar to the current exchange rate in each period. The quarterly values of the effective exchange rates obtained in this way are set out in Annex 4.

\section{Conclusion}

24. In principle, the procedure followed to determine the choice of a weighting system used to calculate relative indicators of competitiveness and of effective exchange rates will depend on the analyses that are to be made with these tools. Cur purpose here was to propose weights which could be used to measure overall trade competitiveness so as to gauge the relative competition engaged in by the partner countries on their markets. We tried in this paper to take account of this aspect while at the same time attempting to satisfy three other criteria at least: first, simplicity of calculation method and utilisation (in particular, the same approach was followed for relative cost and price indicators and effective exchange rates); second, ease of comprehension and interpretation of the weights obtained; third and last, transparency and availability to our numerous correspondents from Member couritry private and public institutions. Several calculation methods could have been used. The one described here seemed to us to satisfy the criteria set out above. 


\section{NOTES}

1. $\bar{i}$ is excluded from the calculation since a country does not compete with itself.

2. For further details on the choice of indicators of competitiveness, see "The International Competitiveness of Selected OECD Countries", OECD Eccnomic Outlook Occasional Studies, July 1978. 
$-13-$

ANNEX 1 


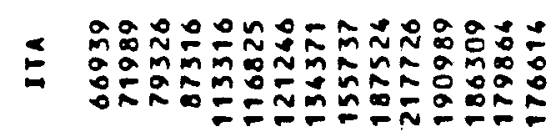

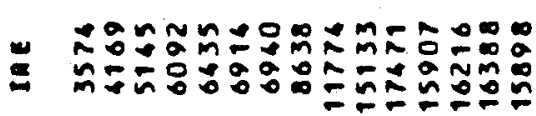

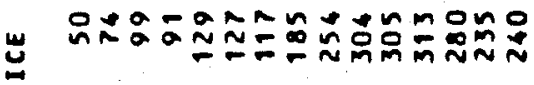

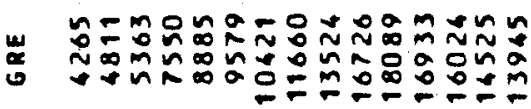

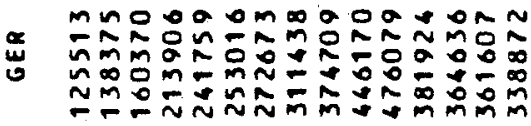

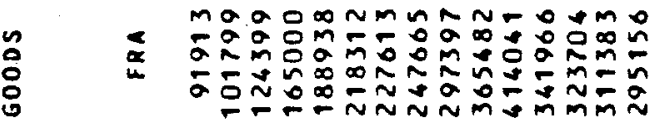

ㅇ -moOmaOOMNONONO

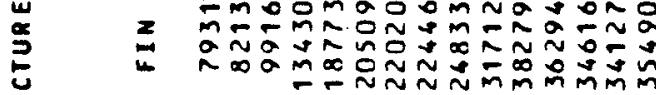

这全

茫

$z^{k}$

0
0
$\vdots$
$\vdots$
0
0
0

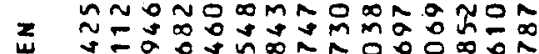

岁

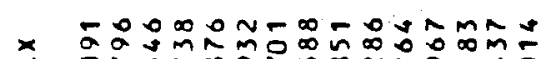

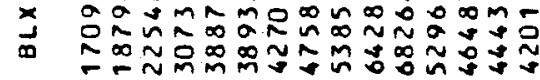

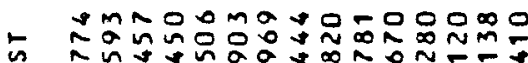

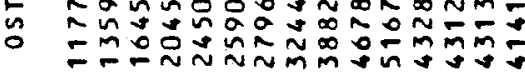

:

i

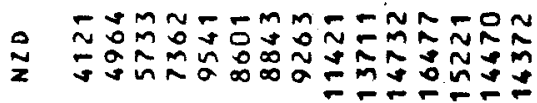

-

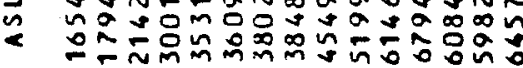

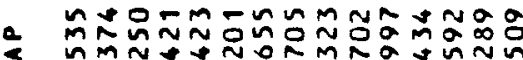

$\left\{\begin{array}{c}0 \\ 0 \\ 0\end{array}\right.$

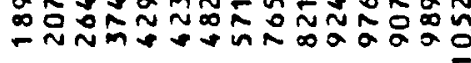

× s

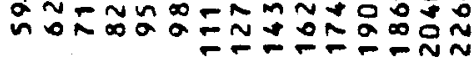

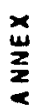

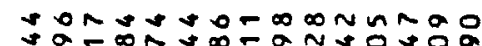

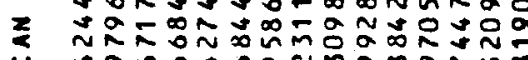

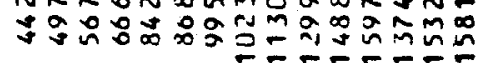

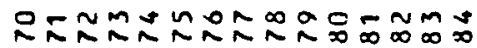

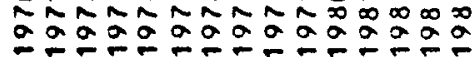

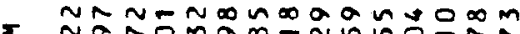

x

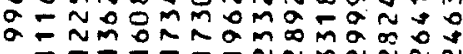

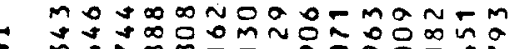

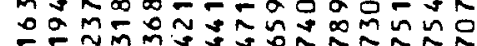

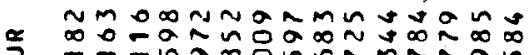

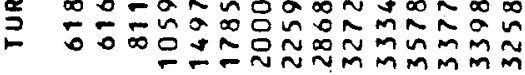

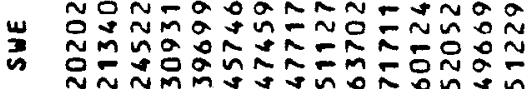

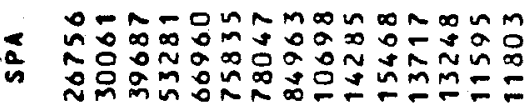

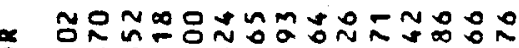
\& N

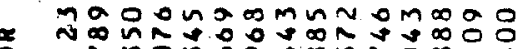

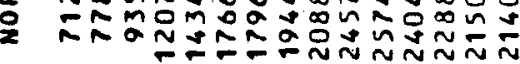

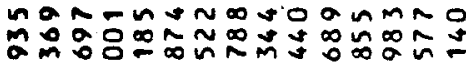

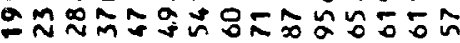

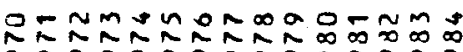

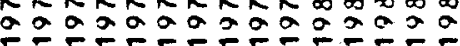


$-15-$

ANNEX 2 


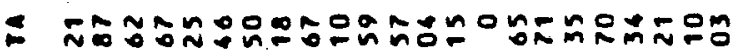

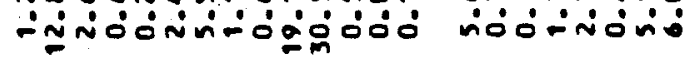

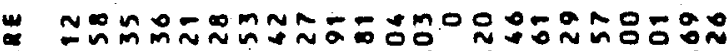

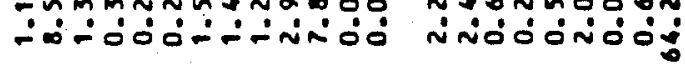

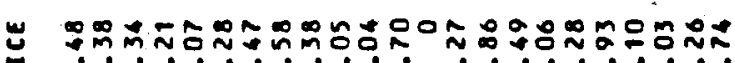

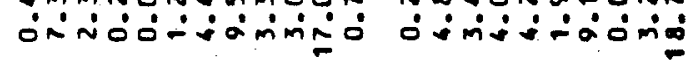

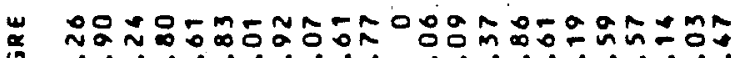

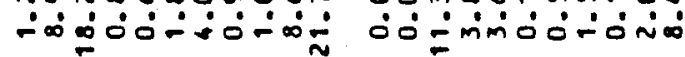

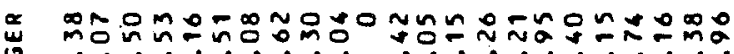

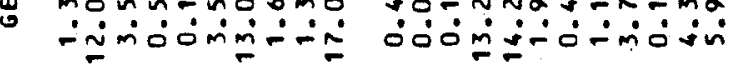

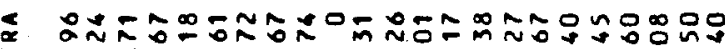

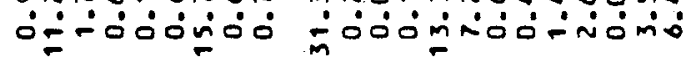

Z

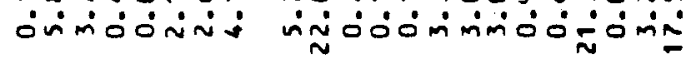

Z

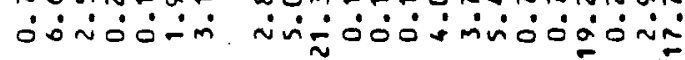

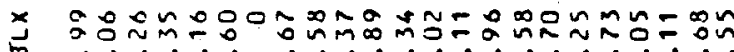

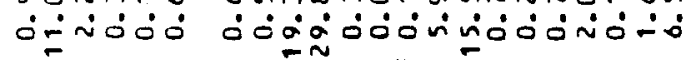

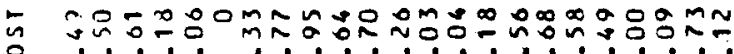

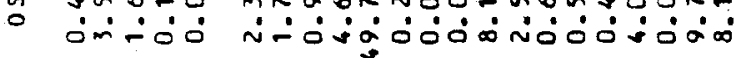

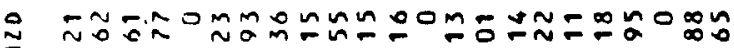

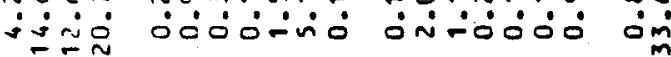

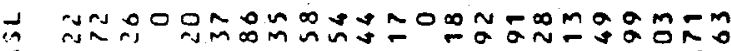

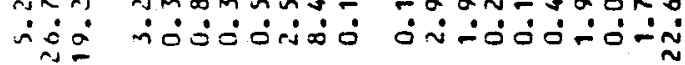

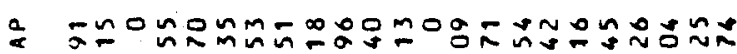

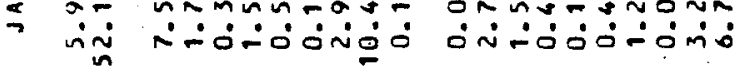

๕

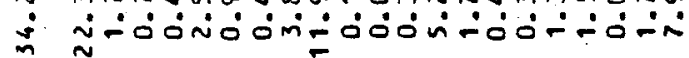

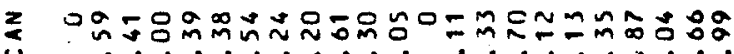

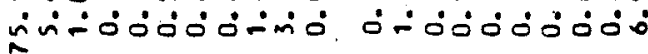

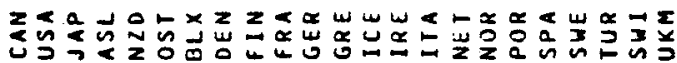

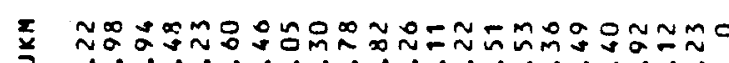

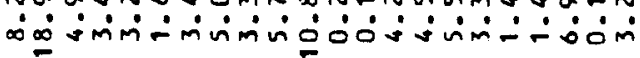

Z

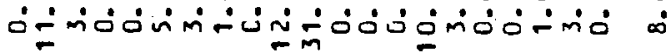

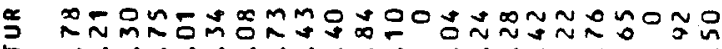

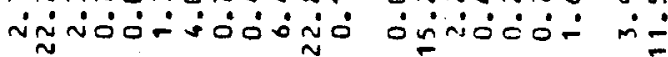

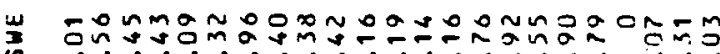

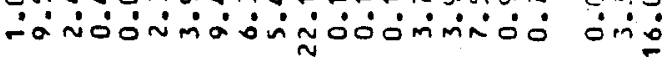

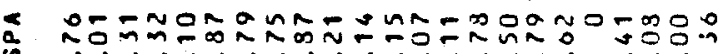

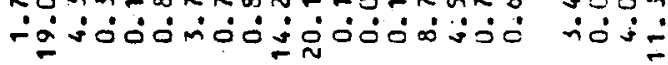

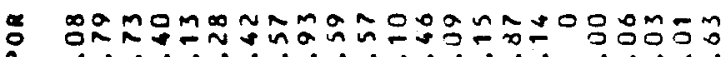

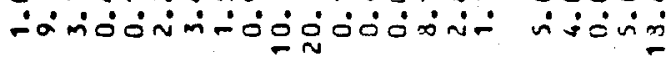

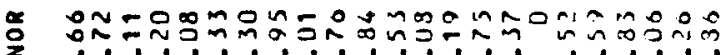

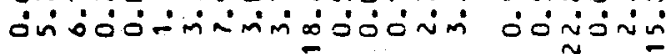

๘

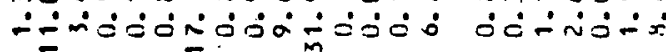

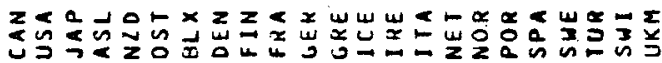




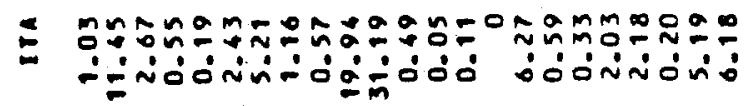

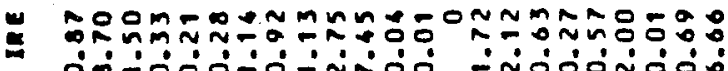

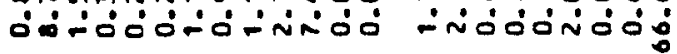

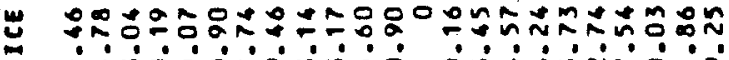

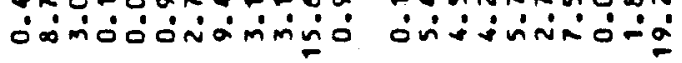

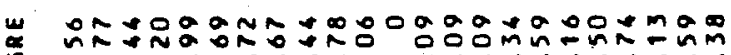

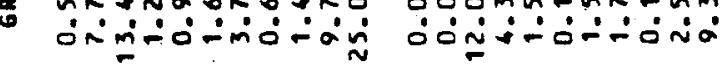

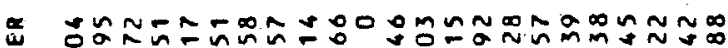

o dindoñ

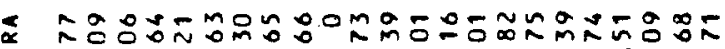

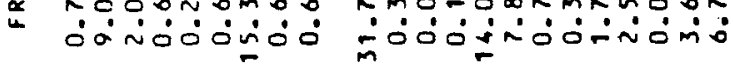

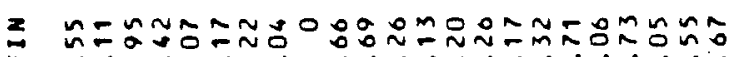

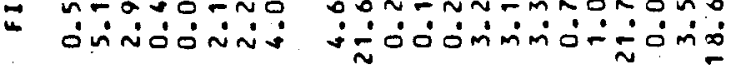

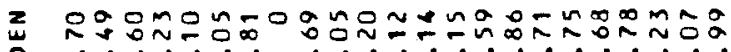

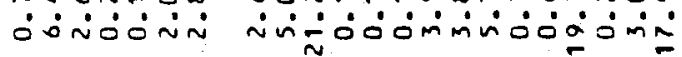

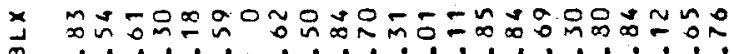

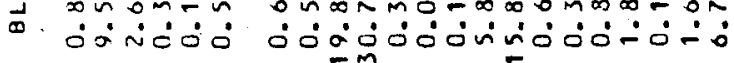

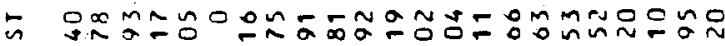

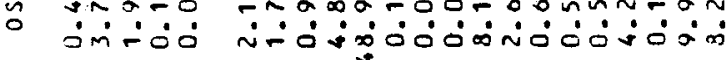

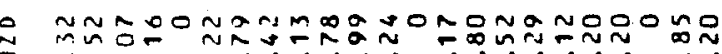

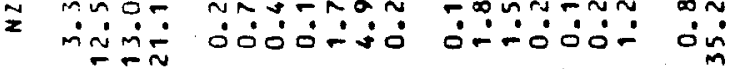

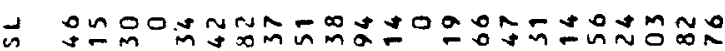

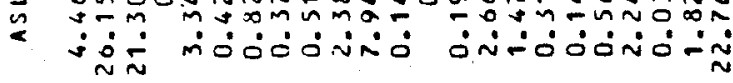

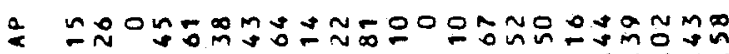

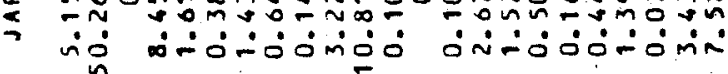

$z$
$x$
w
$z$
$z$

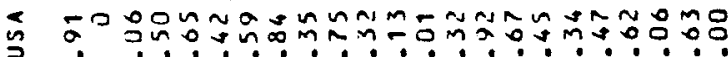

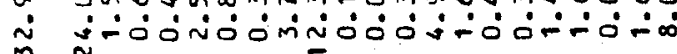

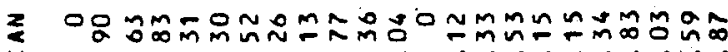

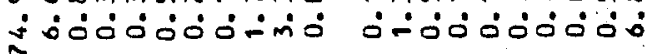

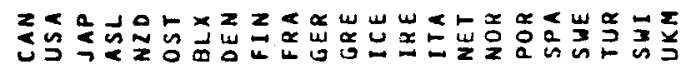

₹

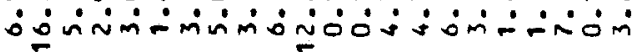

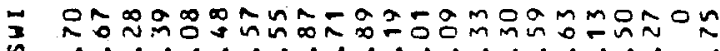

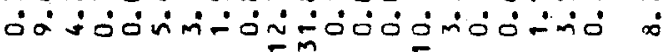

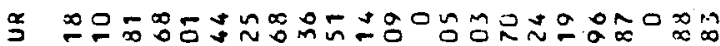
ن

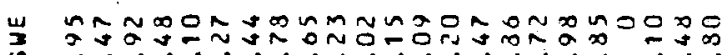

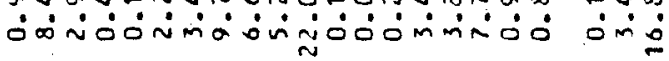

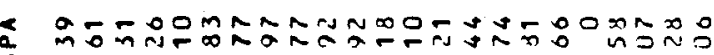

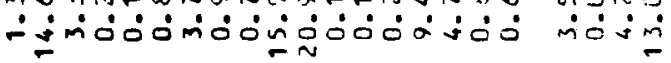

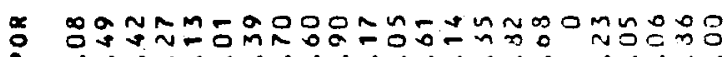

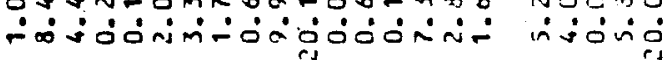

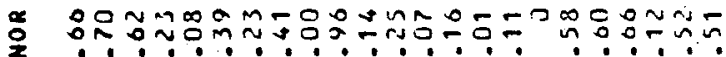

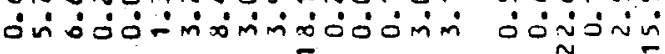

ป

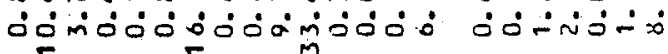

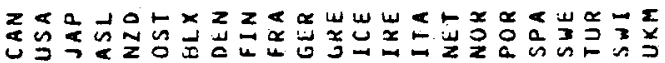


E mogkñnn

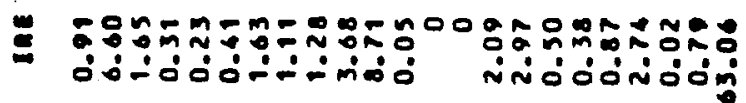

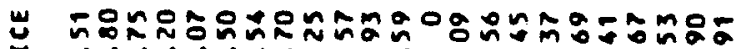

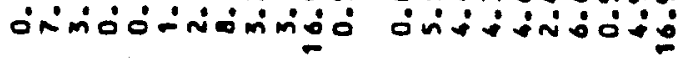

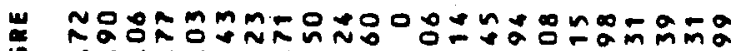

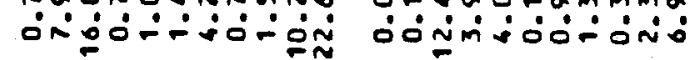

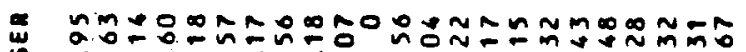

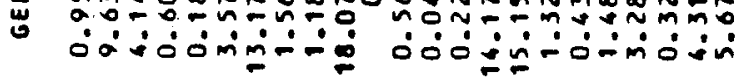

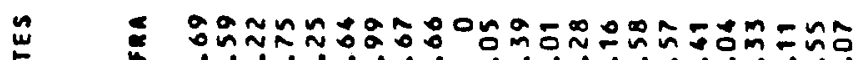

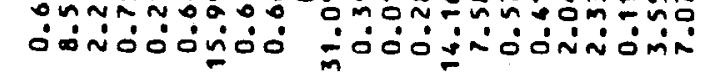

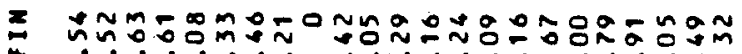

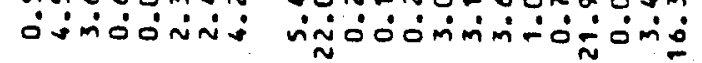

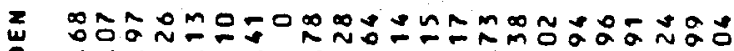

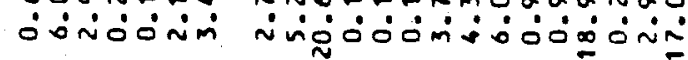

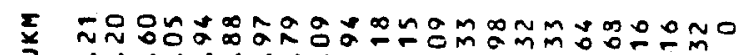

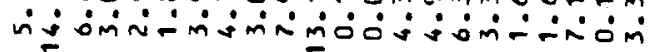

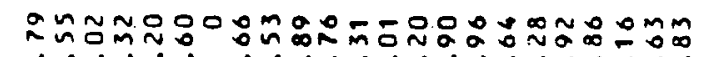

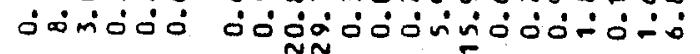

క

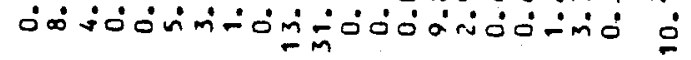

Ł Ominó

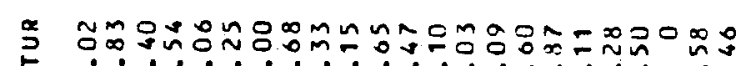
-nmod-

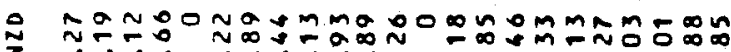

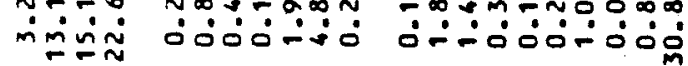

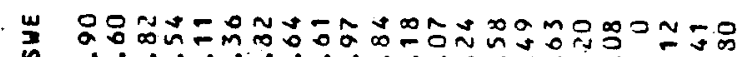

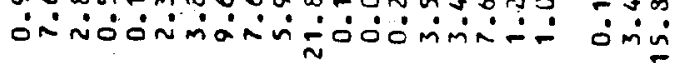

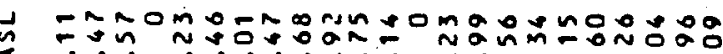

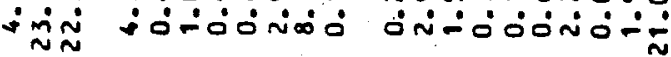

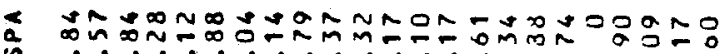

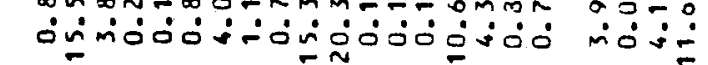

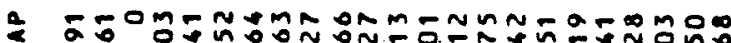

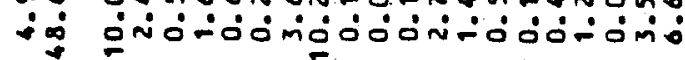

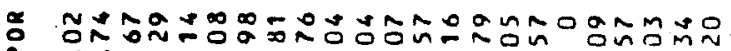

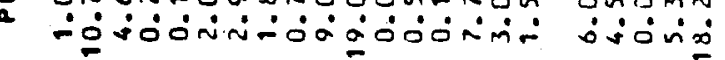

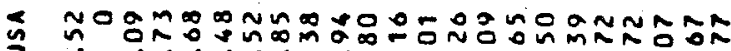
m

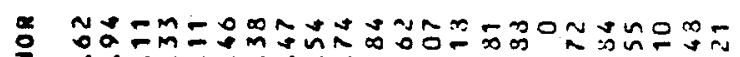

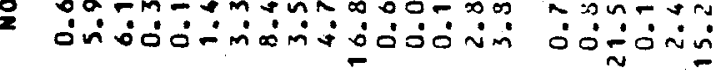

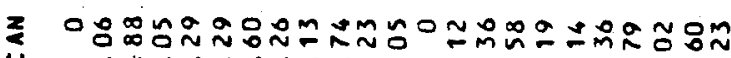

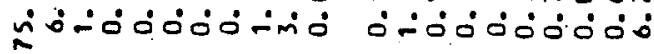

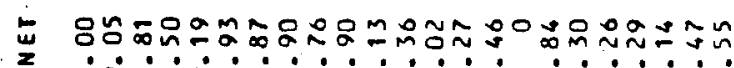

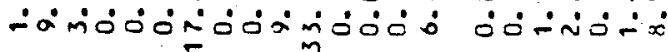




\section{$E$}

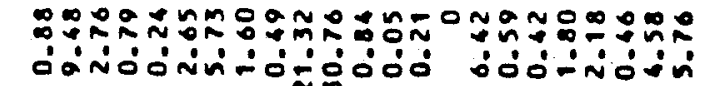

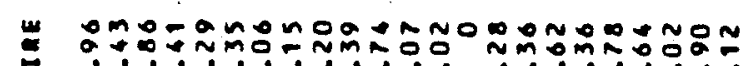

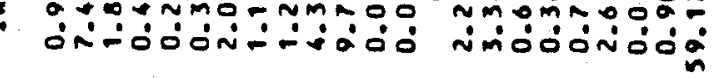

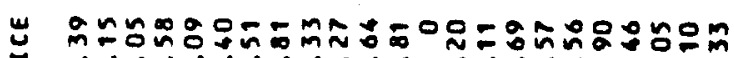

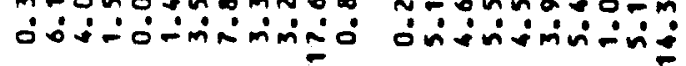

ะ

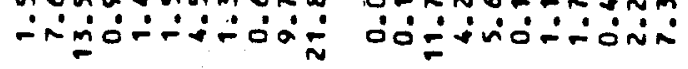

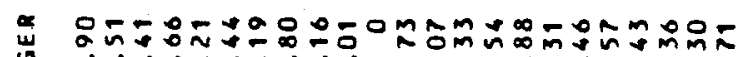

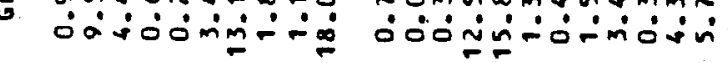

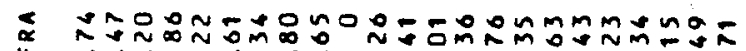

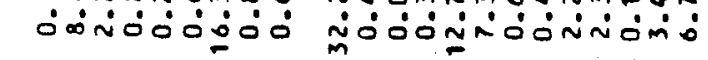

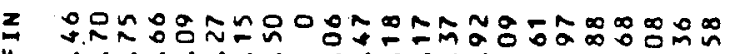

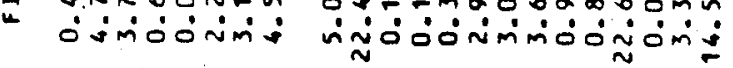

z

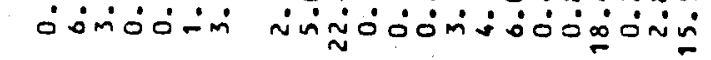

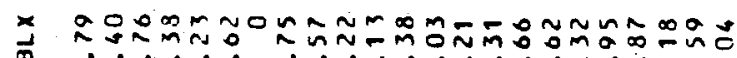

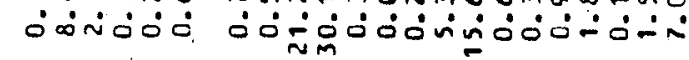

๒

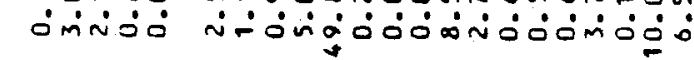

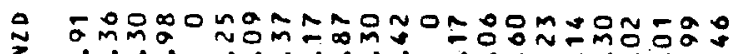

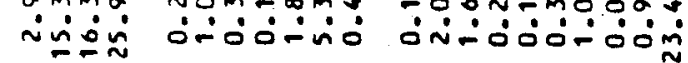

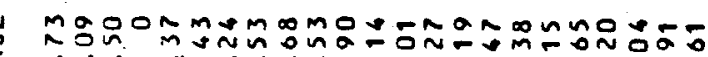
is

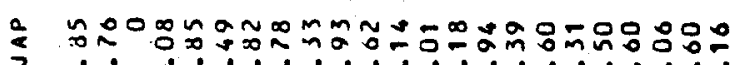

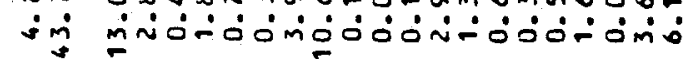

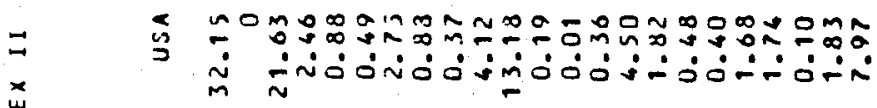

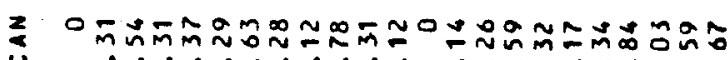

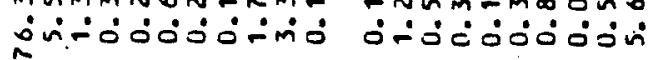

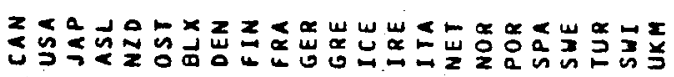

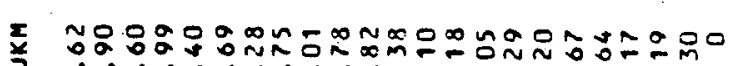

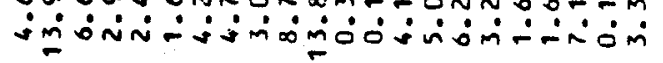

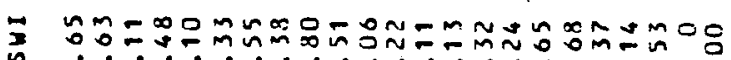

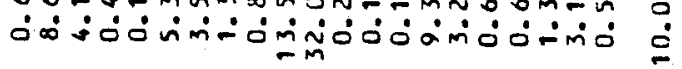

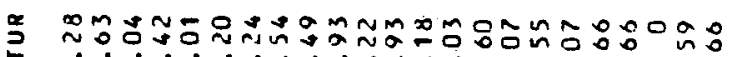
- $=$ ó

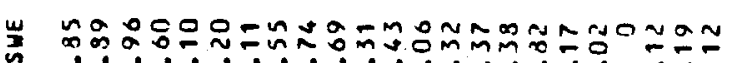

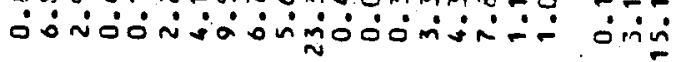

\&

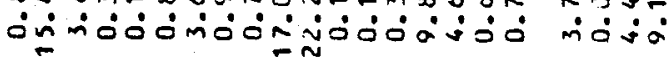

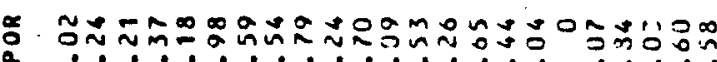

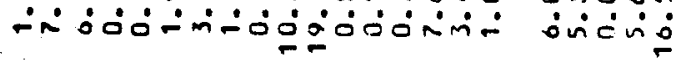

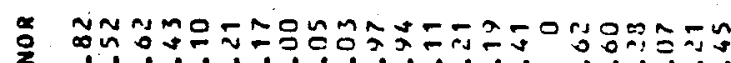

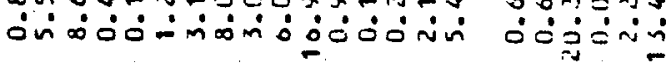

—

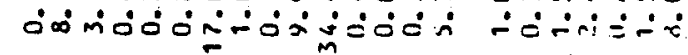

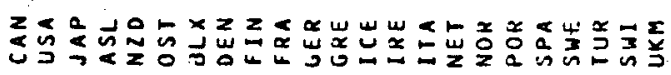




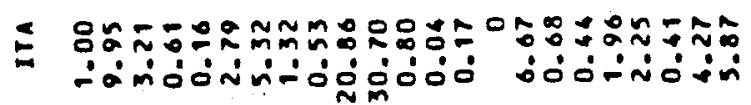

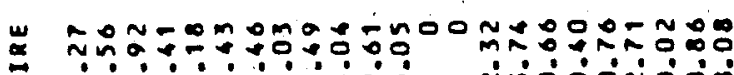

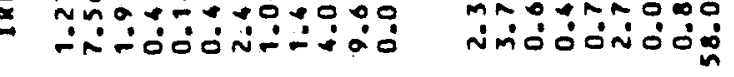

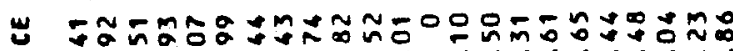

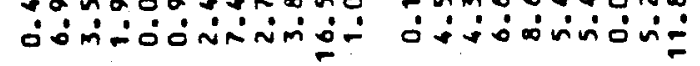

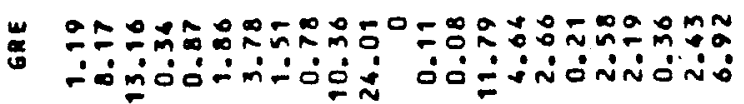

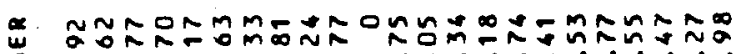

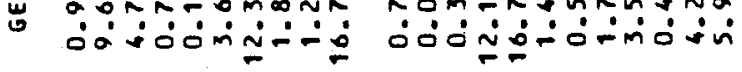

ะ

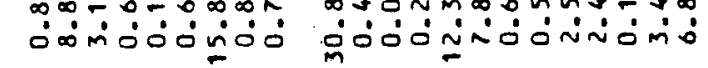

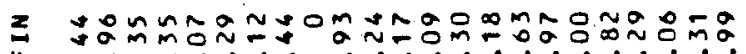

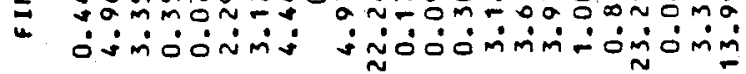

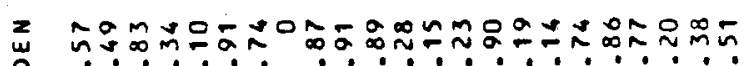

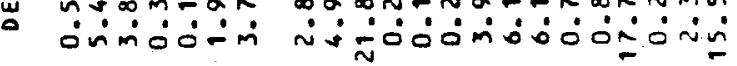

メ

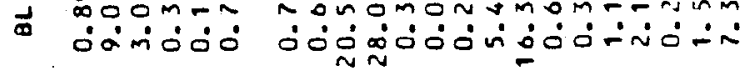

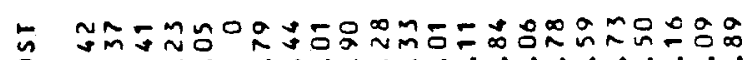

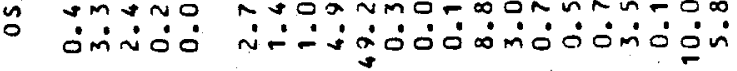

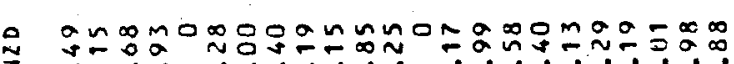

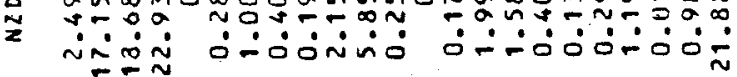

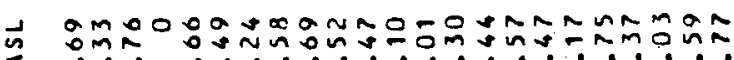

m

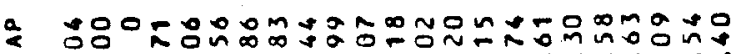

$\rightarrow$ nin

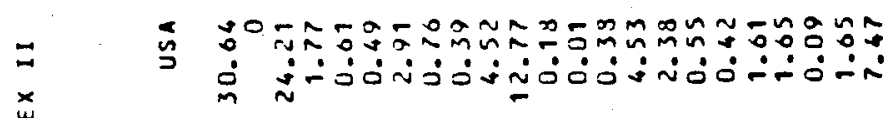

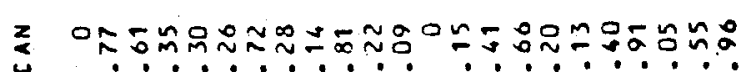

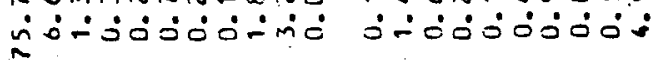

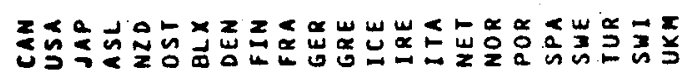

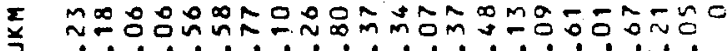

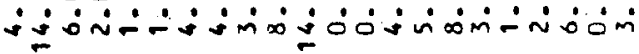

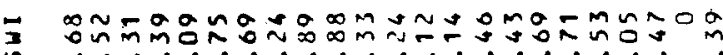

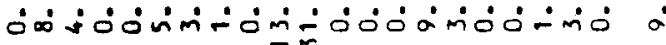

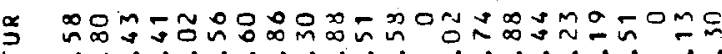

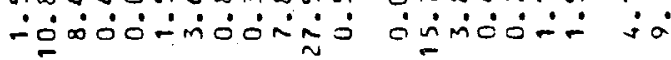

纨

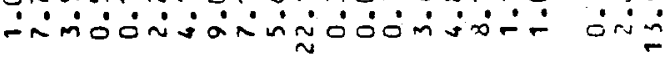

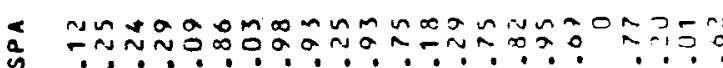

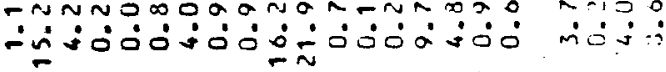

×

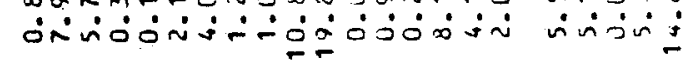

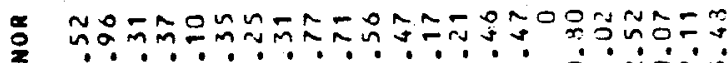

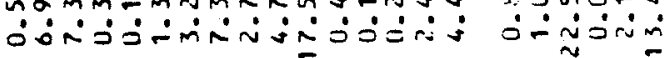

-

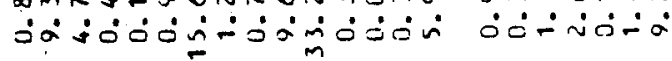

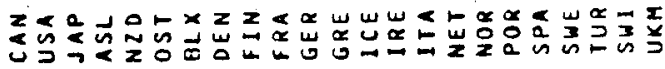


-

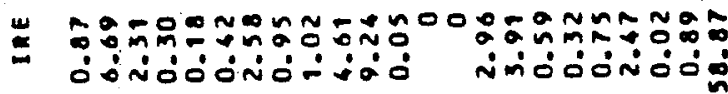

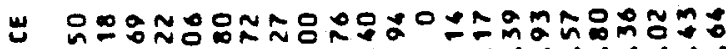

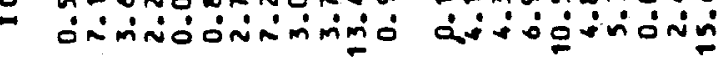

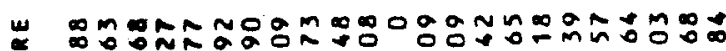

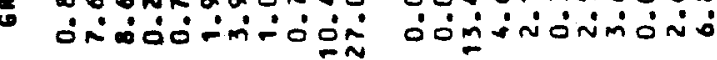

品

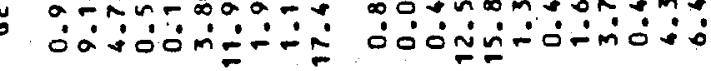

ะ

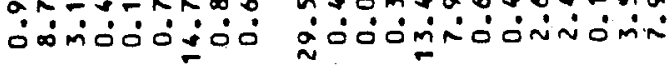

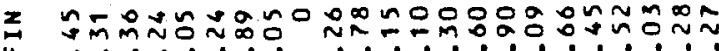

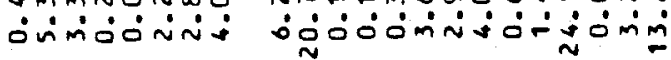

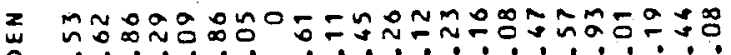

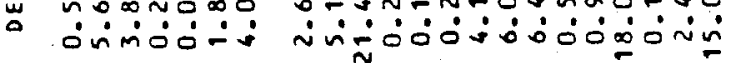

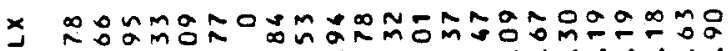

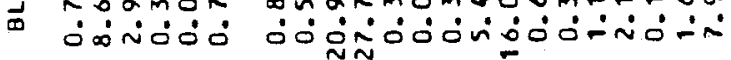

โ

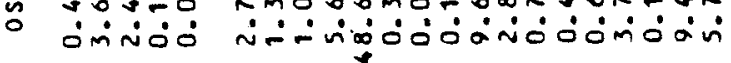

ล ニน์

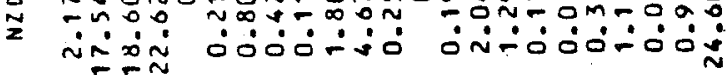

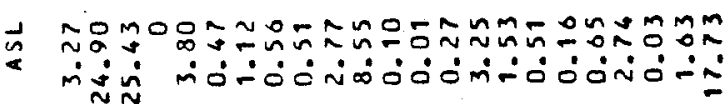

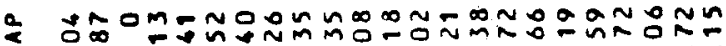

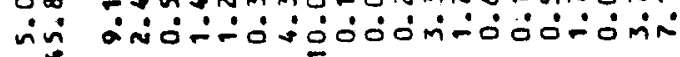

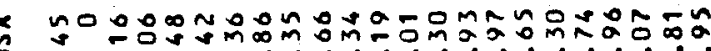
i

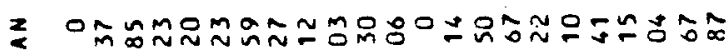

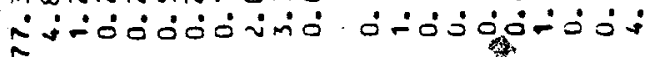

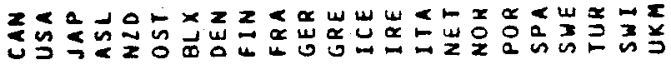

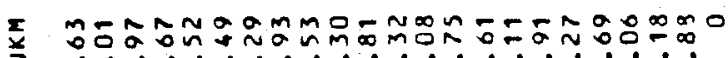

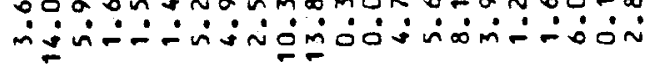

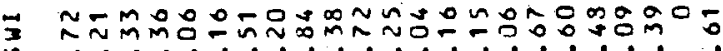

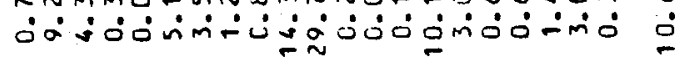

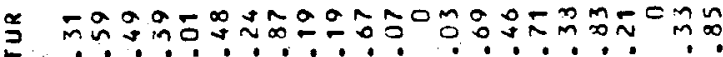

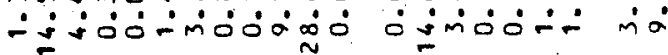

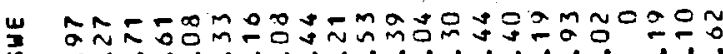

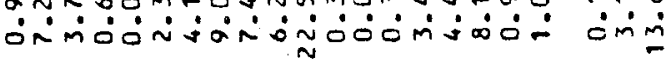

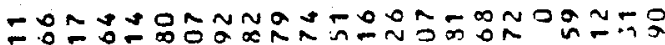

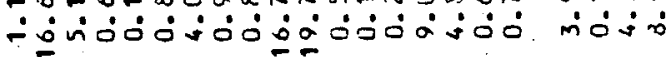

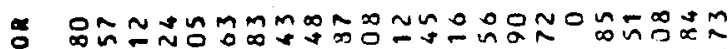

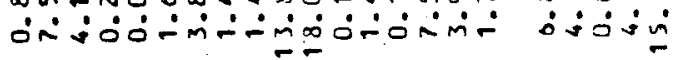

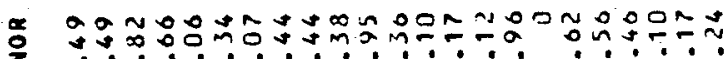

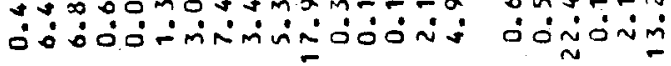

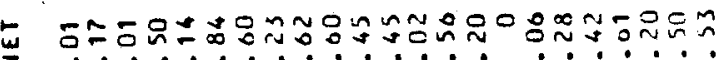

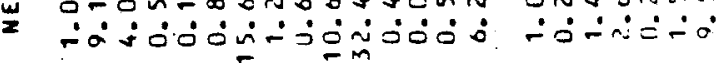

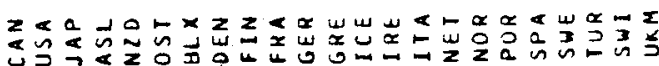




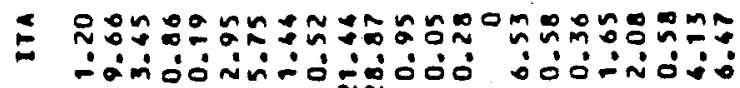

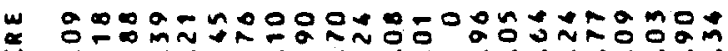

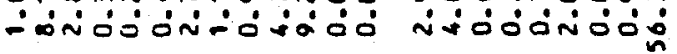

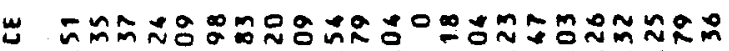

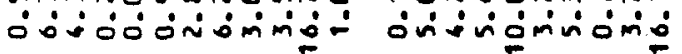

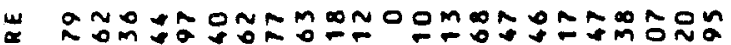

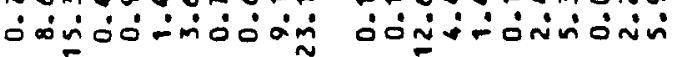

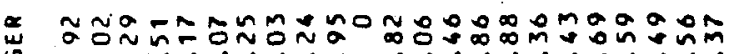

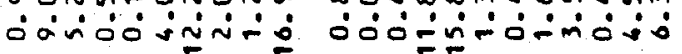

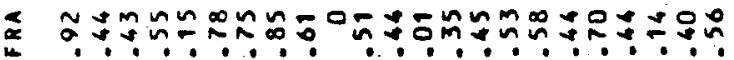

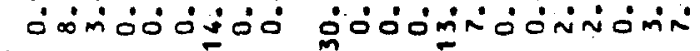

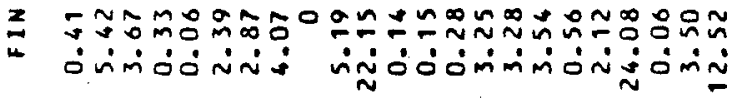

Z ง

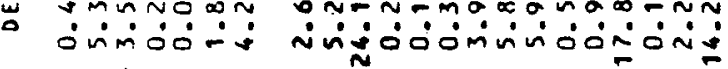

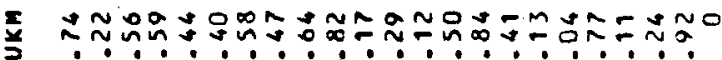

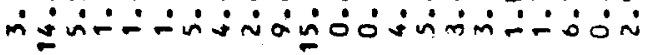

メ

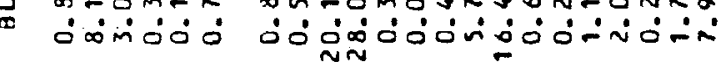

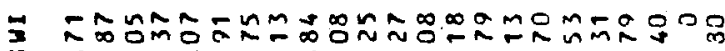

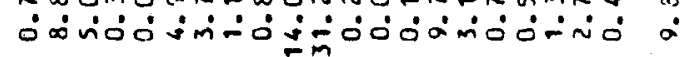

Б

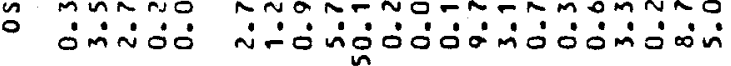

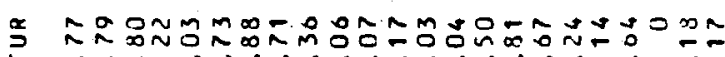

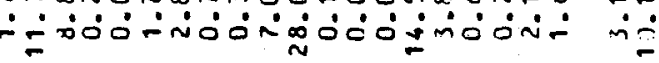

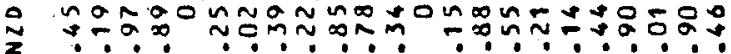

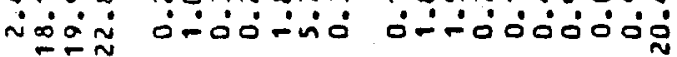

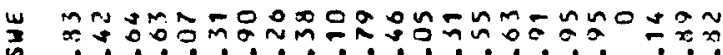

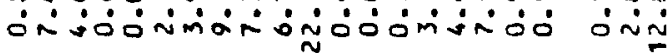

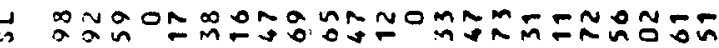

अ

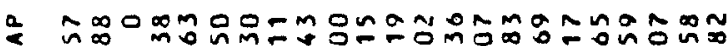

$=$ nُ

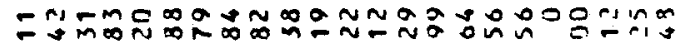

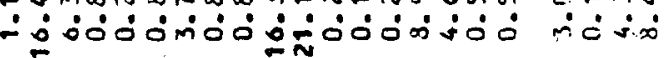

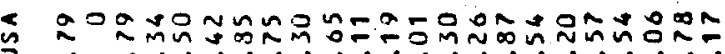

min

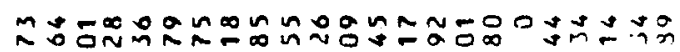

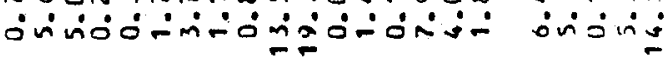

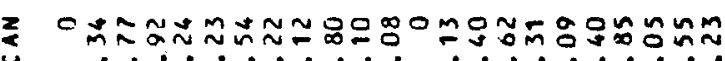

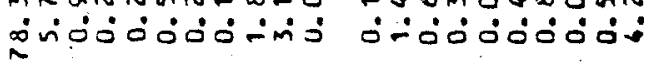

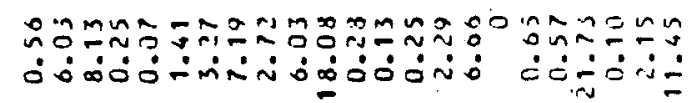

ษ

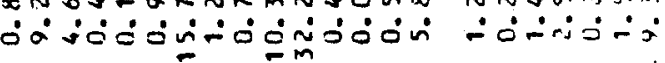

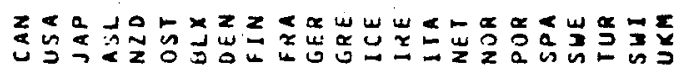

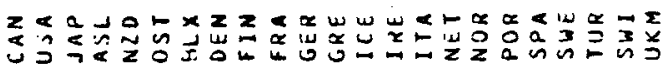




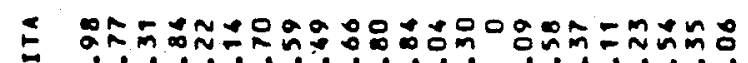

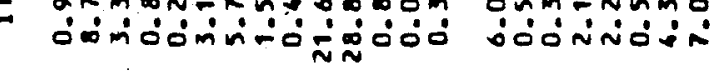

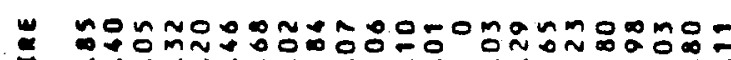

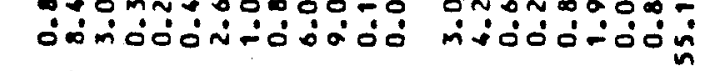

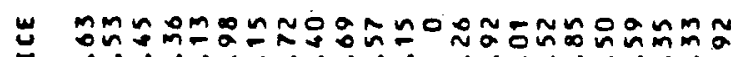

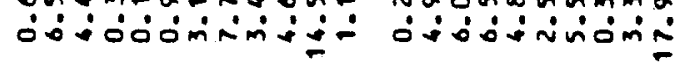

\&

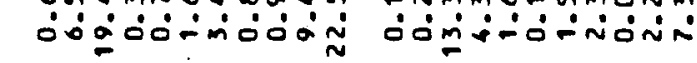

๓

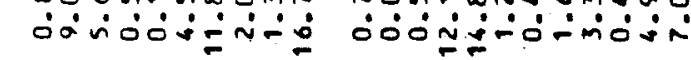

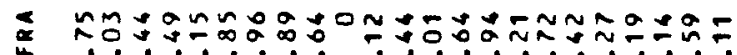

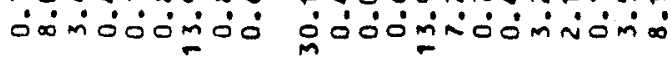

Z Sisóninm

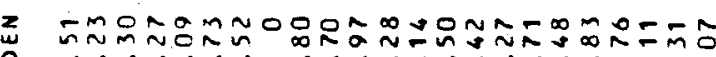

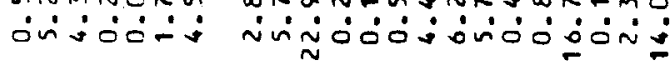

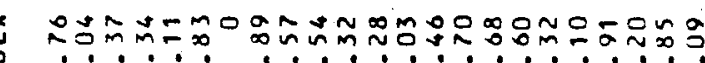

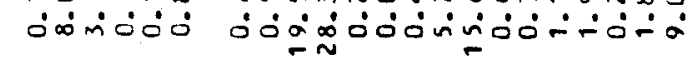

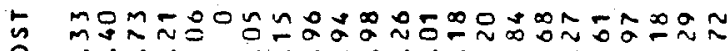

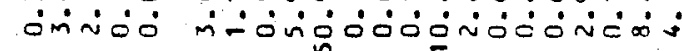

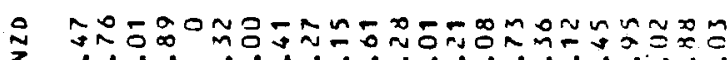

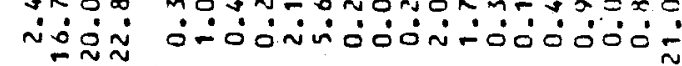

药

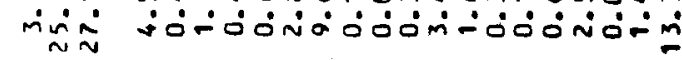

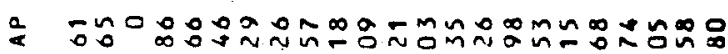

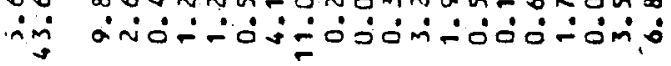

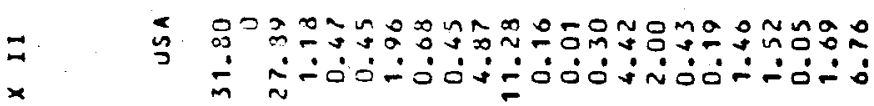

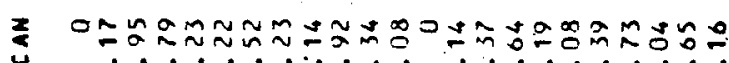

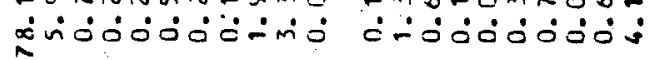

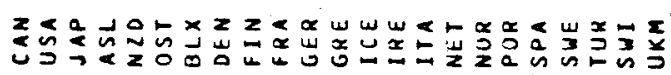

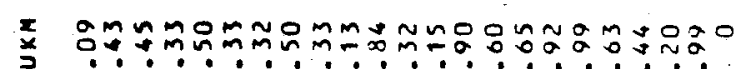

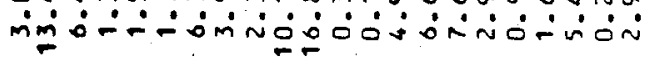

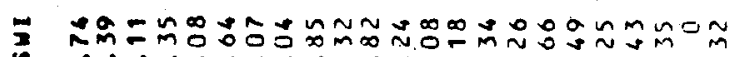

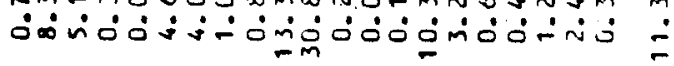

节

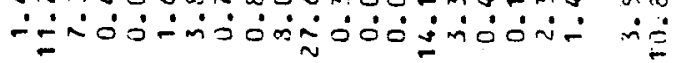

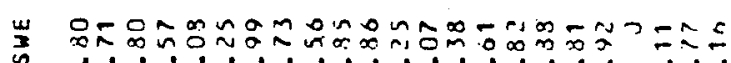

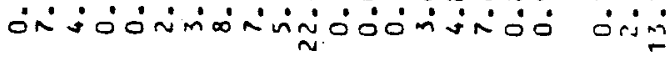

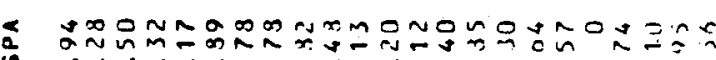

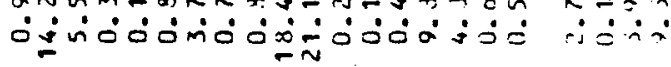

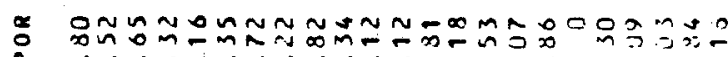

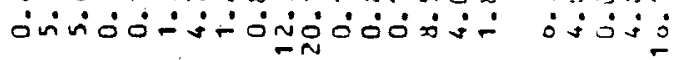

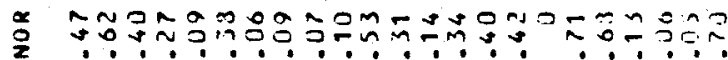

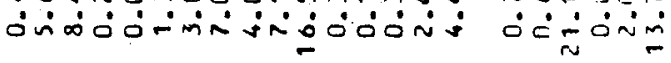

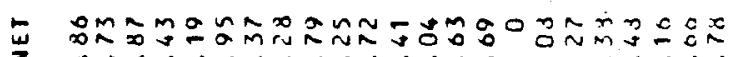

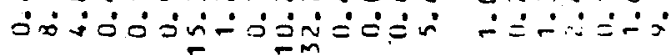

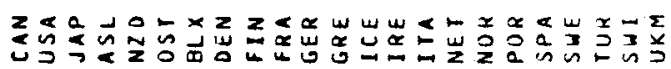




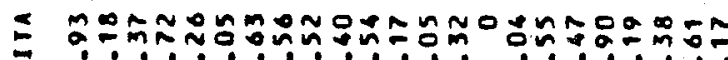

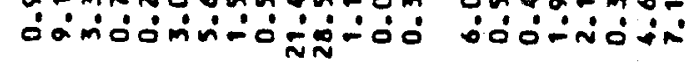

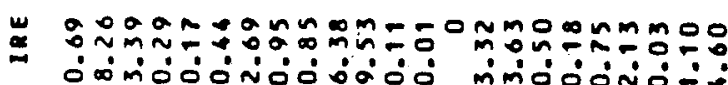

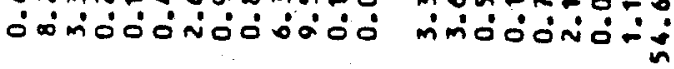

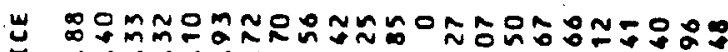

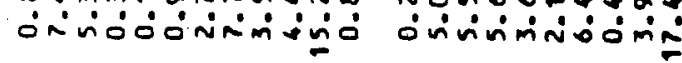

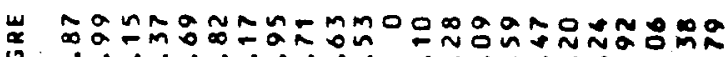

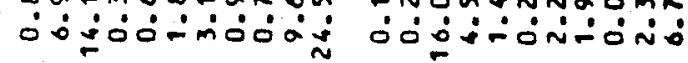

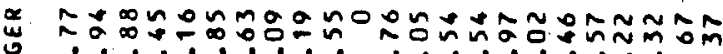

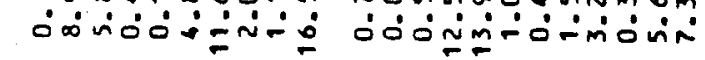

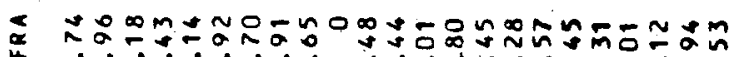
onmosóñó

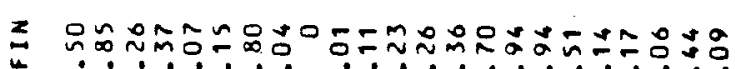

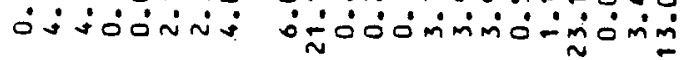

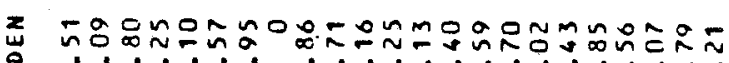

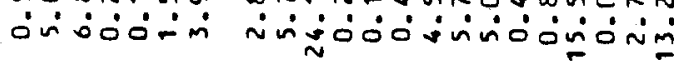

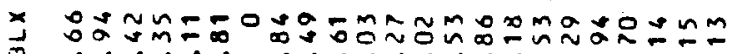

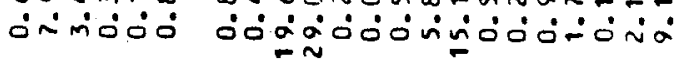

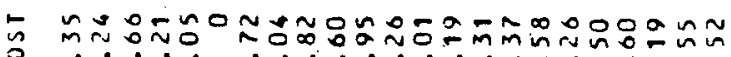

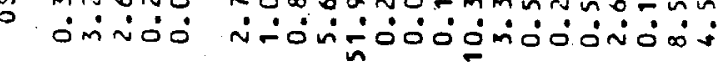

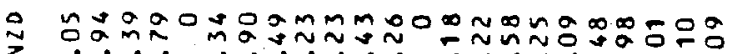

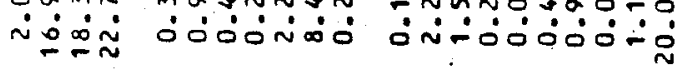

म

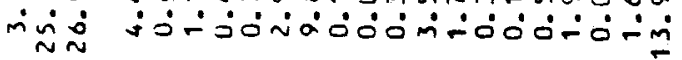

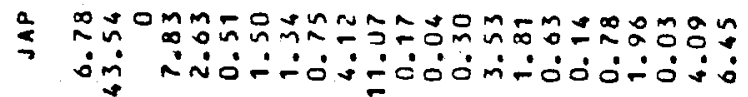

3
$x$
$\frac{x}{2}$
$\frac{2}{2}$

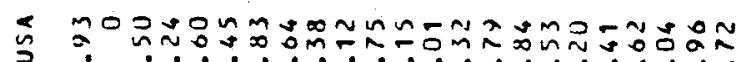
a

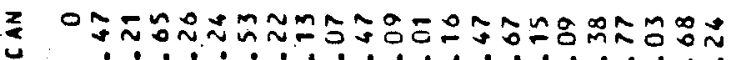

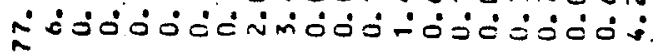

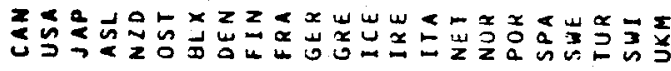

$\stackrel{2}{z}$

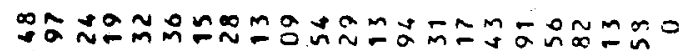

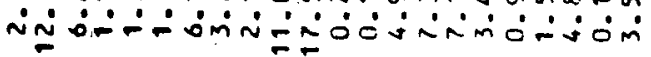

I

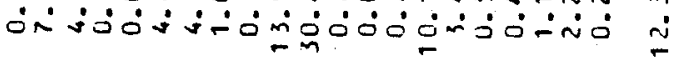

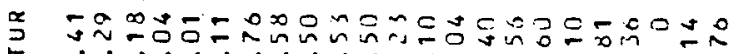

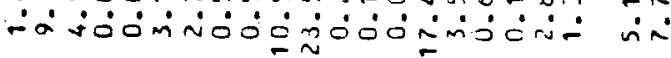

岁

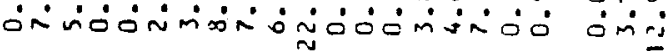

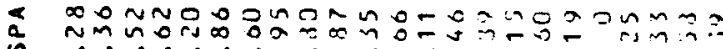

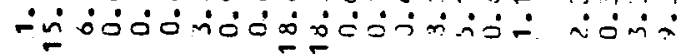

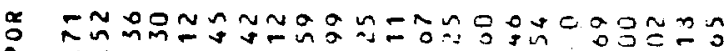

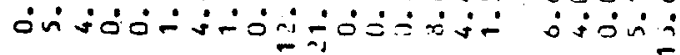

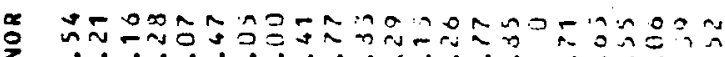

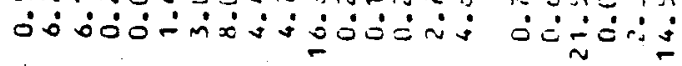

ะ

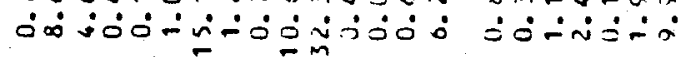

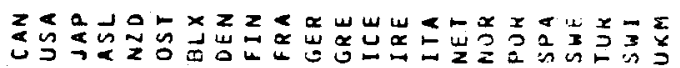




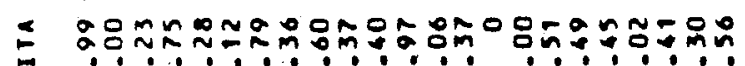

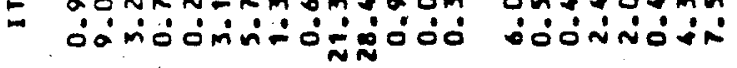

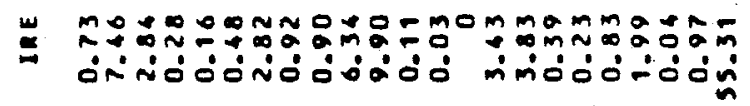

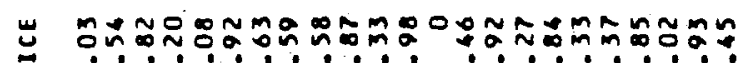

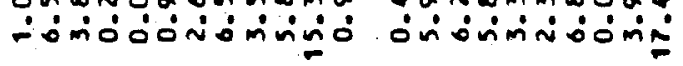

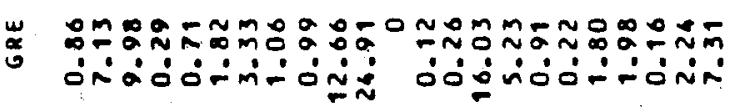

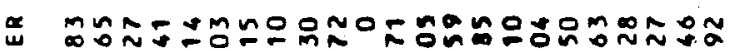

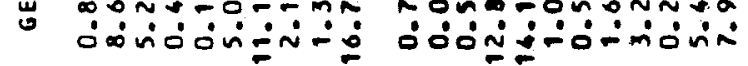

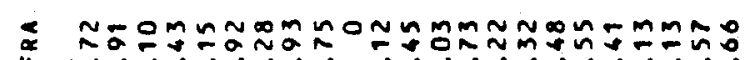

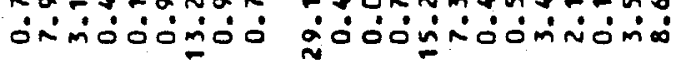

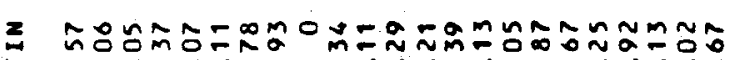

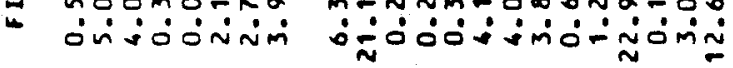

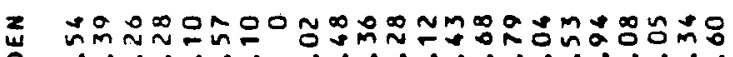

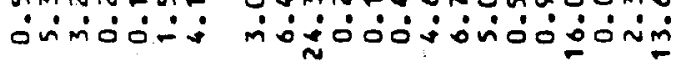

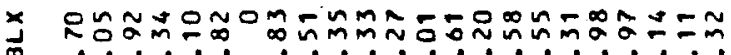

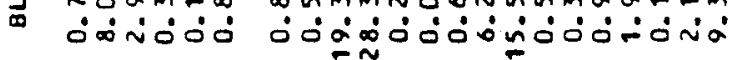

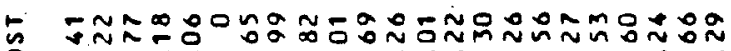

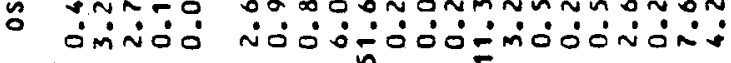

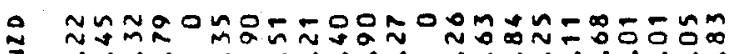

z

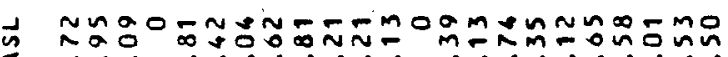

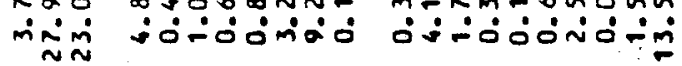

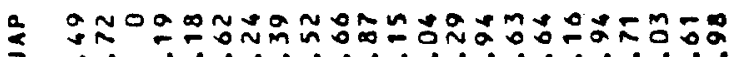

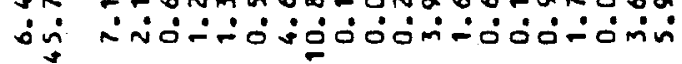

ล

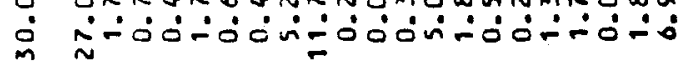

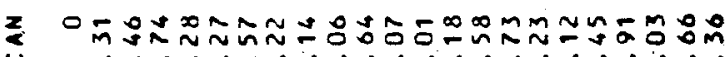

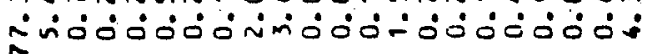

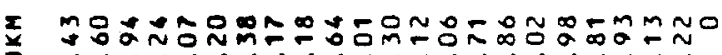
i

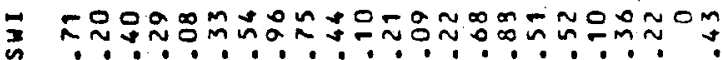

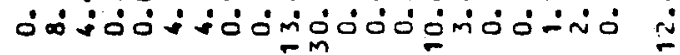

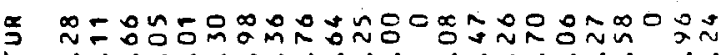

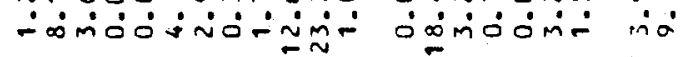

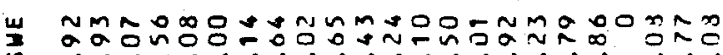

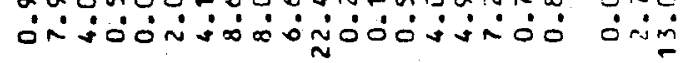

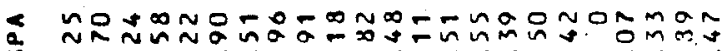

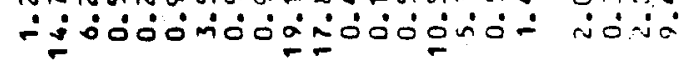

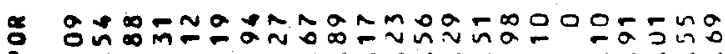

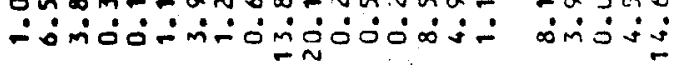

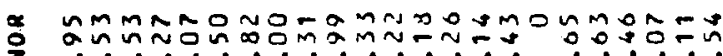

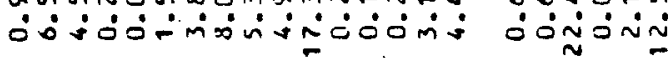

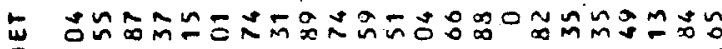

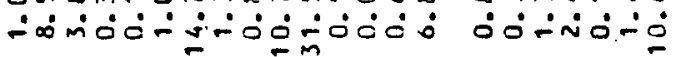

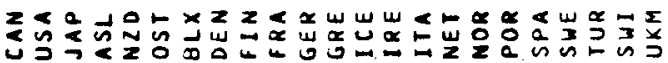




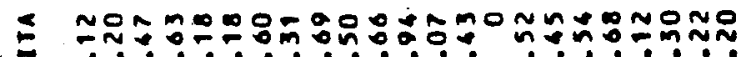

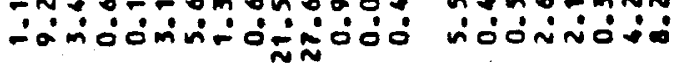

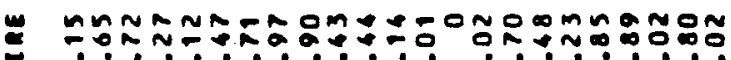

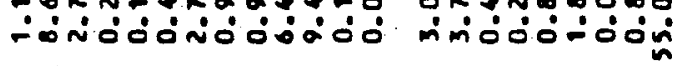

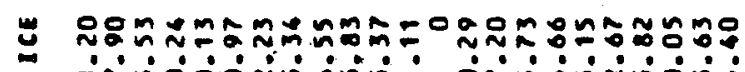

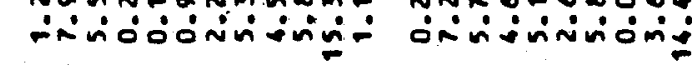

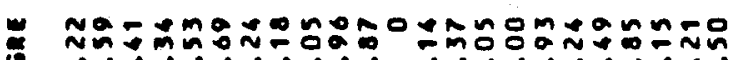

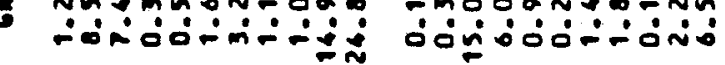

政

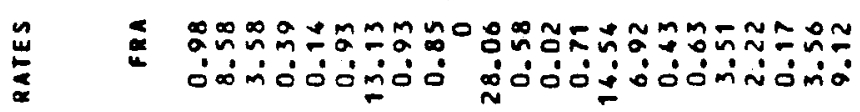

Z

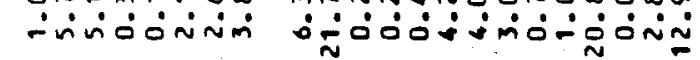

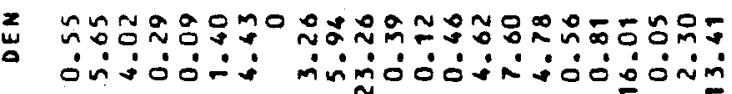

జ

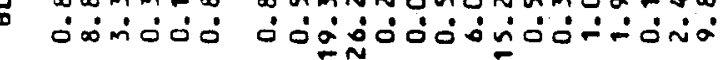

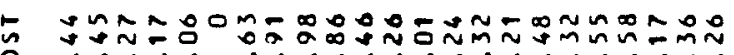

Omíd

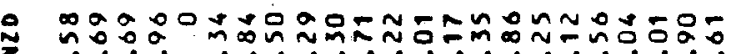

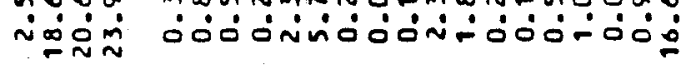

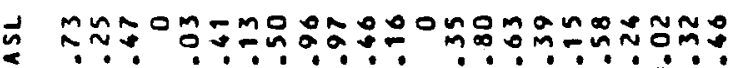

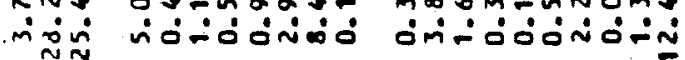

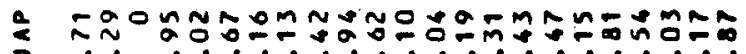

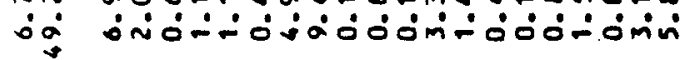

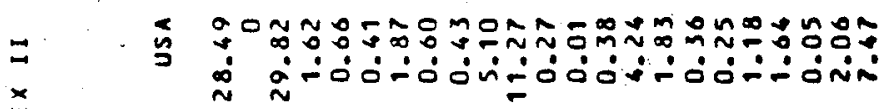

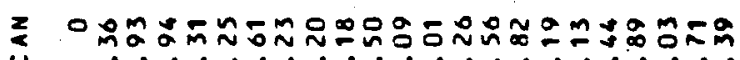

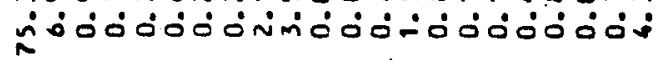

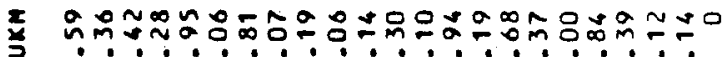

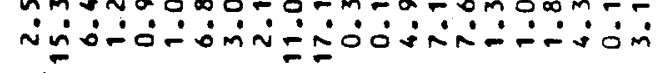

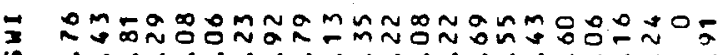

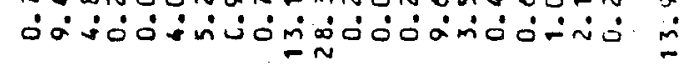

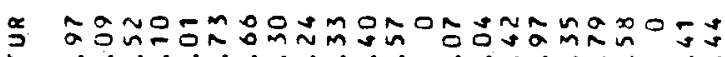

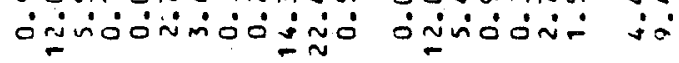

แ

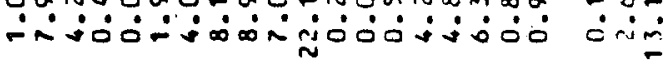

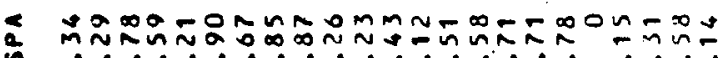

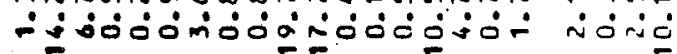

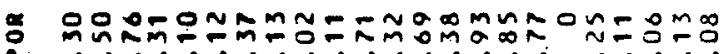

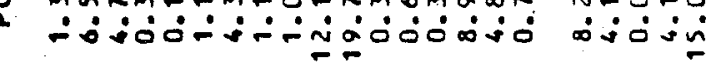

×

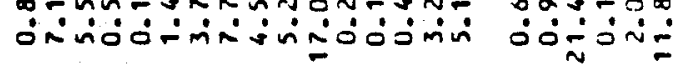

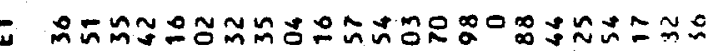

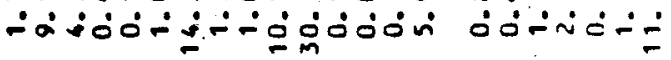


- On

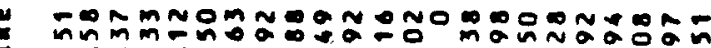

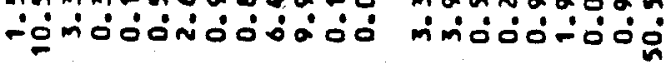

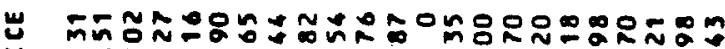

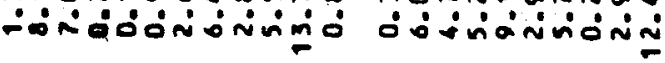

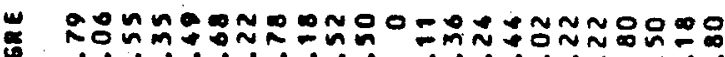

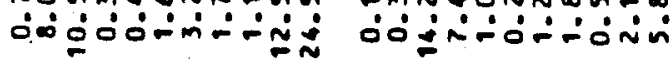

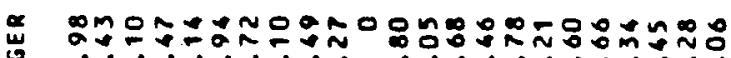

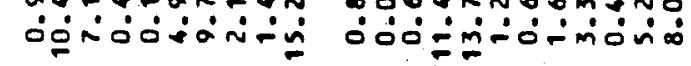

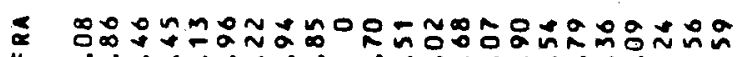

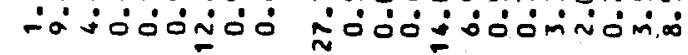

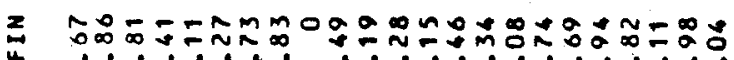

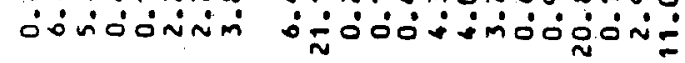

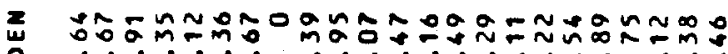

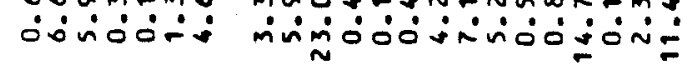

×

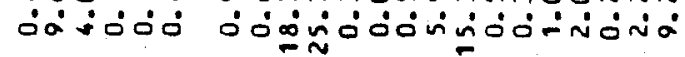

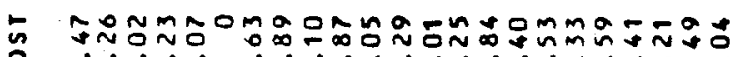

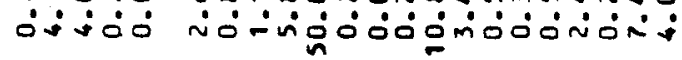

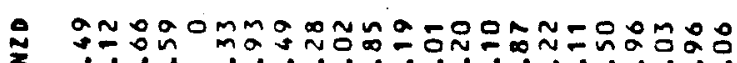
ن

ม

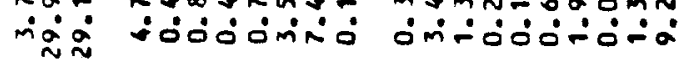

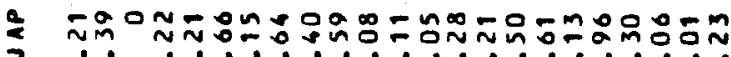

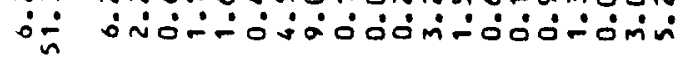

ร

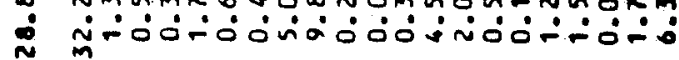

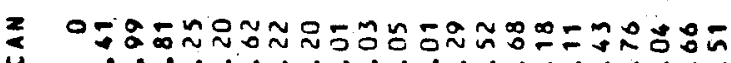

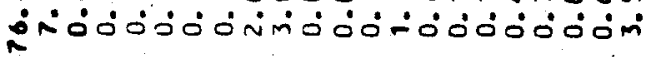

艾

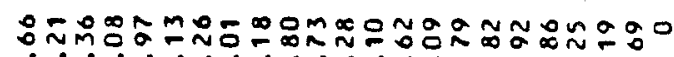

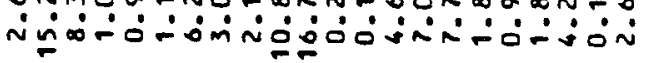

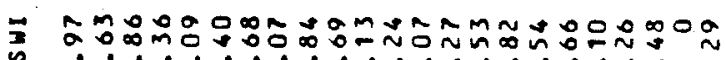

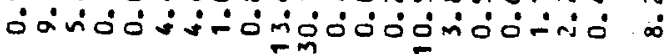

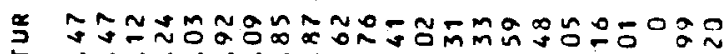

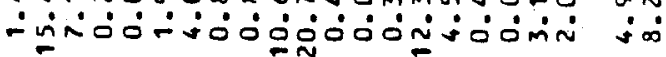

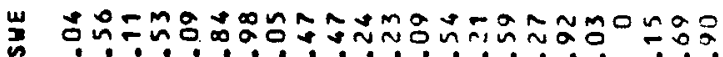

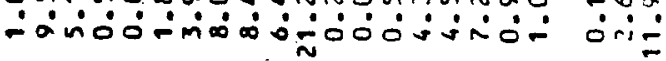

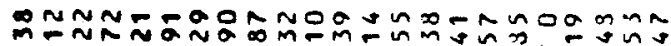

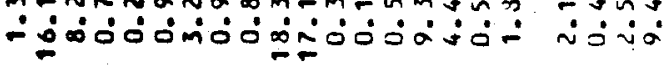

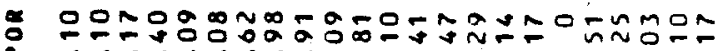

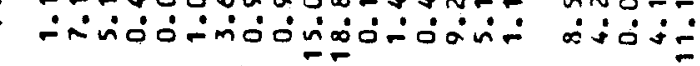

ะ

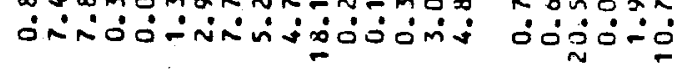

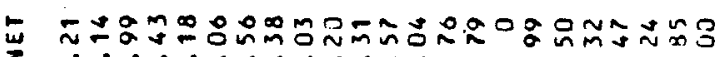

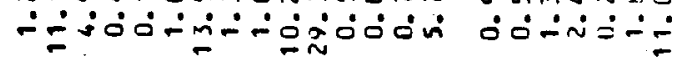

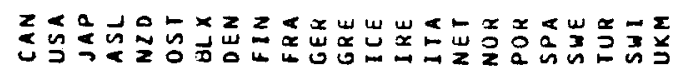


E

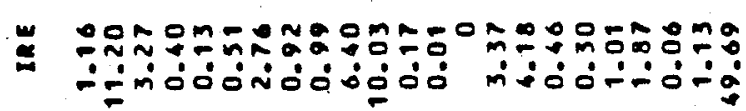

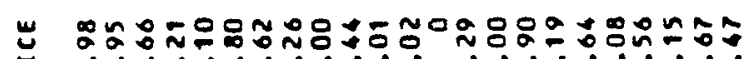

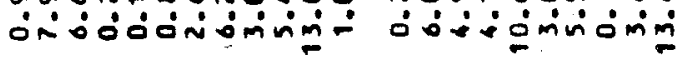

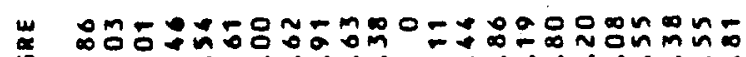

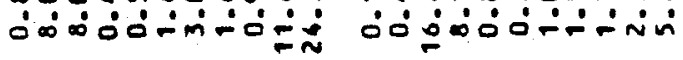

兑

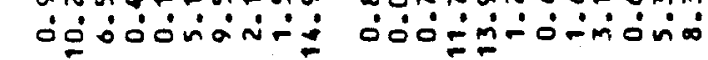

๔

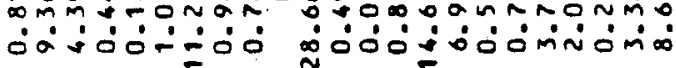

z 워

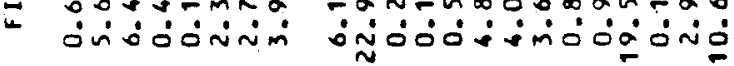

药

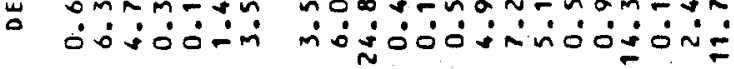

×

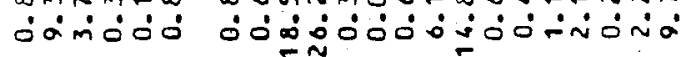

Б

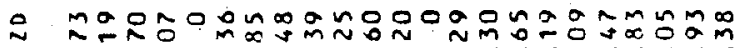

z

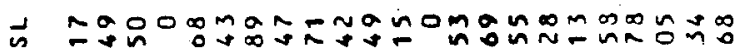

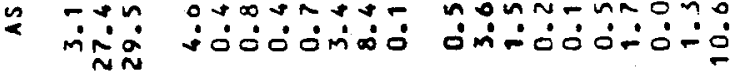

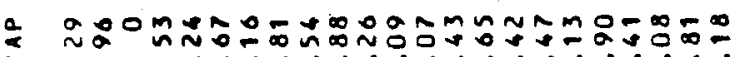

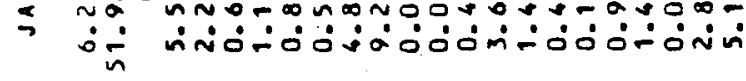

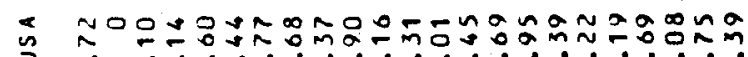

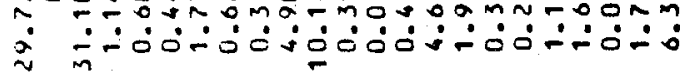

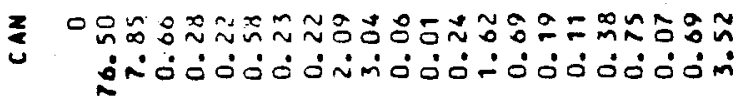

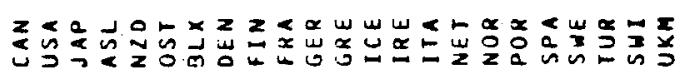

z

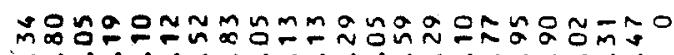

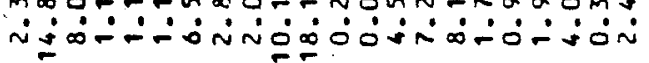

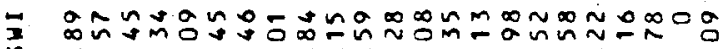

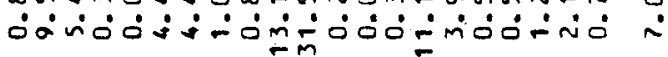

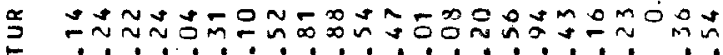

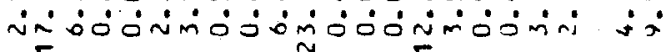

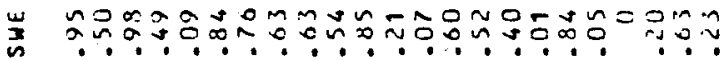

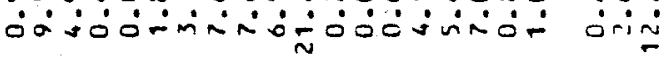

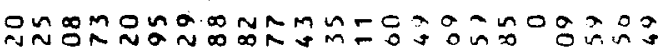
-

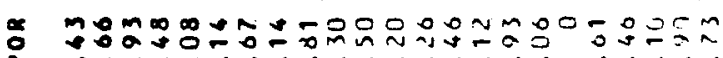

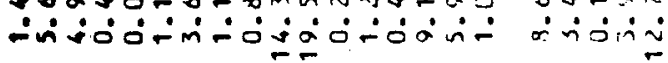

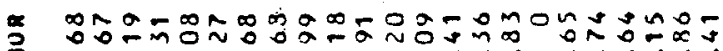

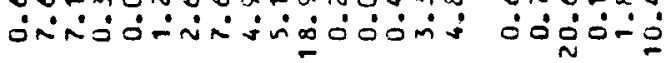

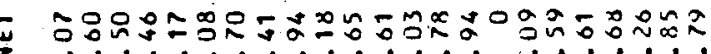

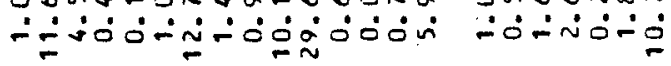

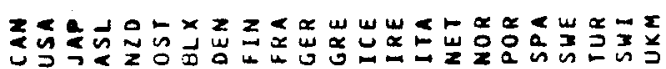




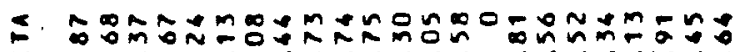

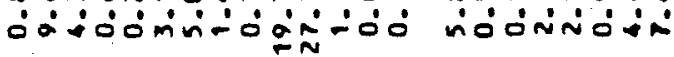

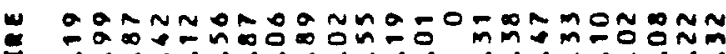

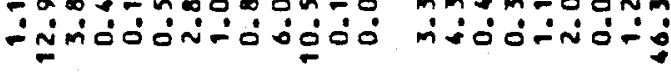

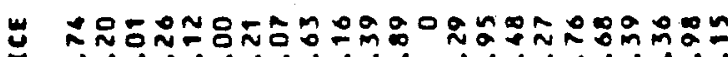

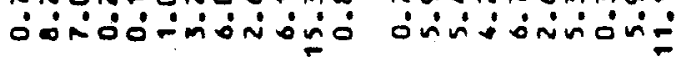

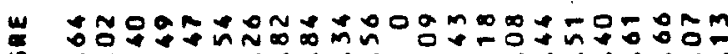

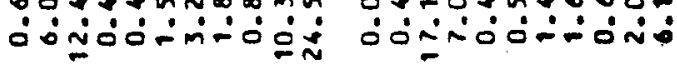

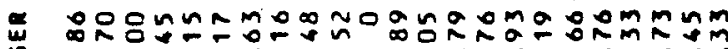

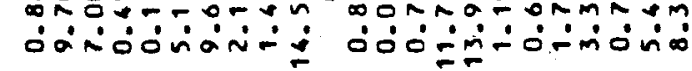

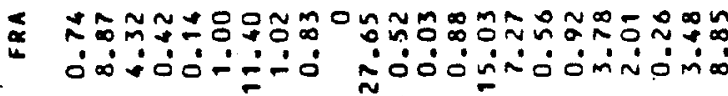

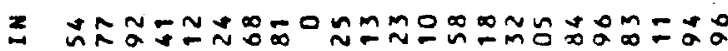

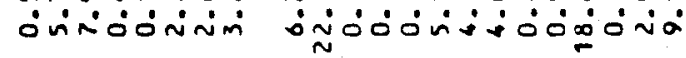

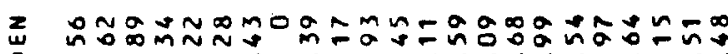

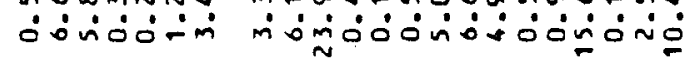

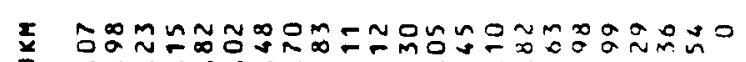

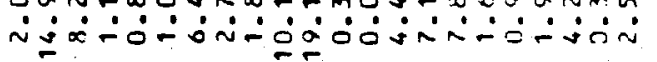

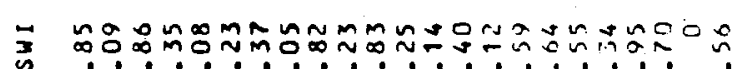

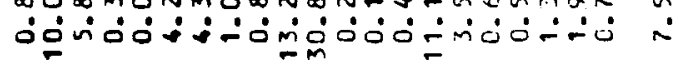

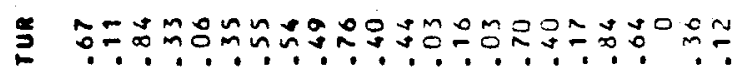

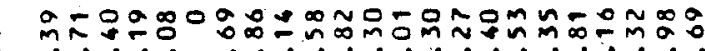

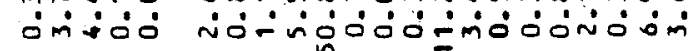

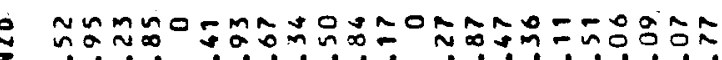

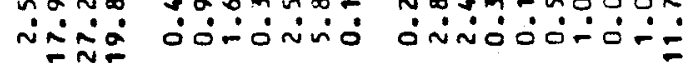

岁

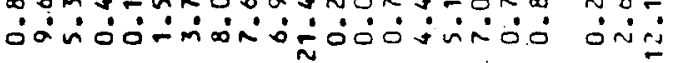

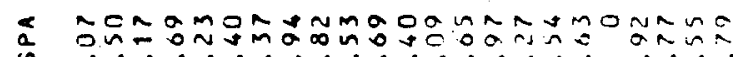

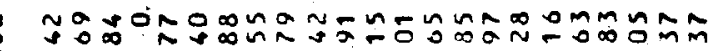
ن

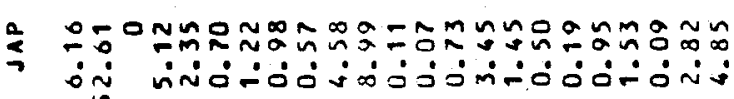

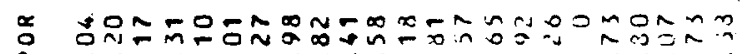

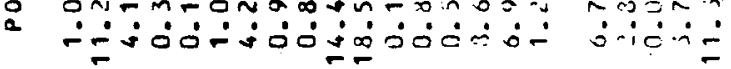

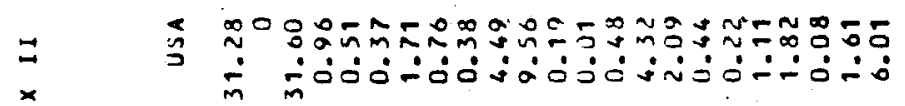

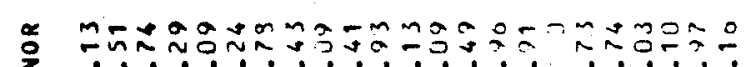

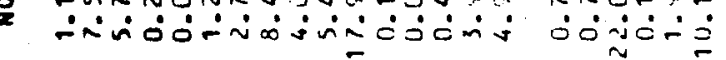

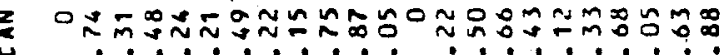

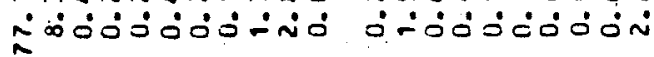

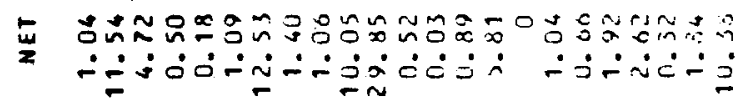




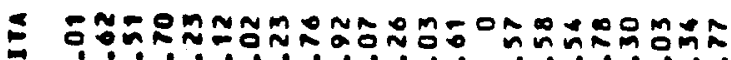

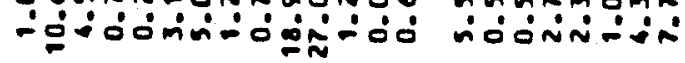

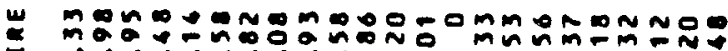

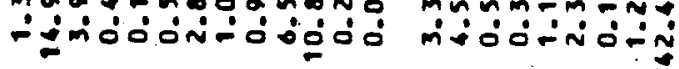

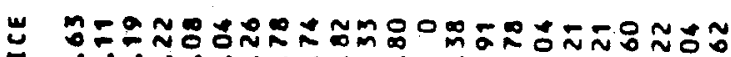

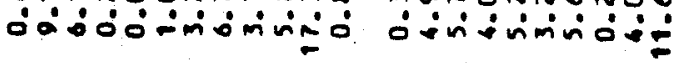

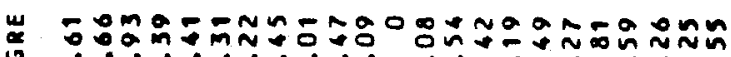

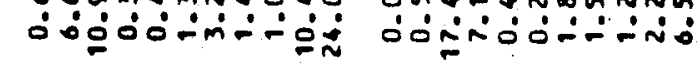

ะ

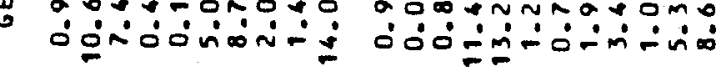

ะ

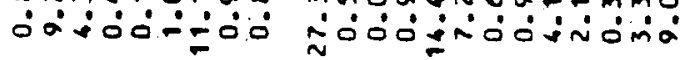

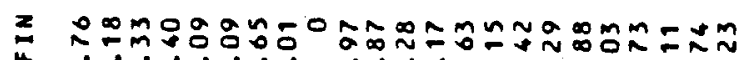

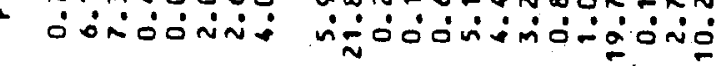

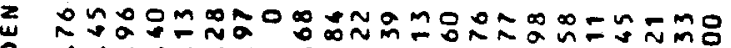

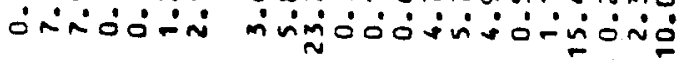

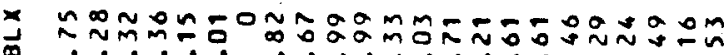

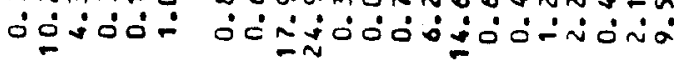

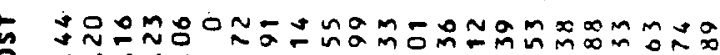

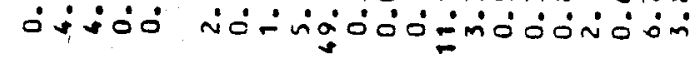

₹

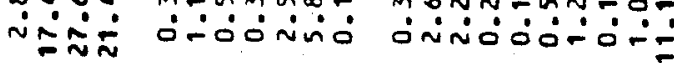

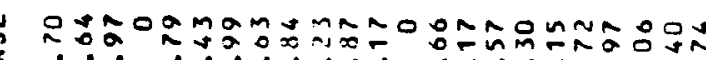

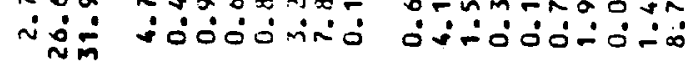

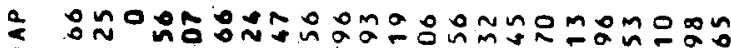

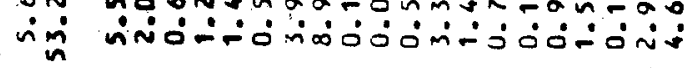

乡

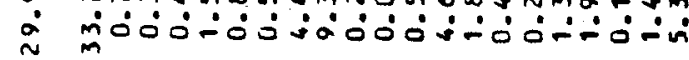

₹

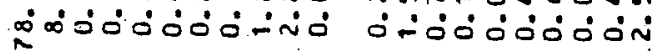

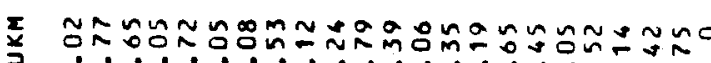

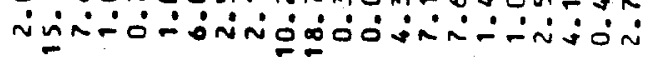

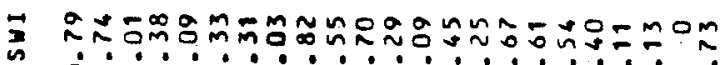

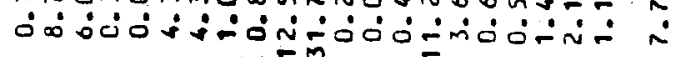

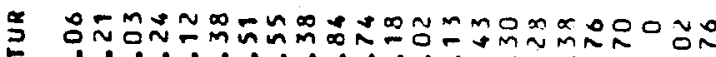

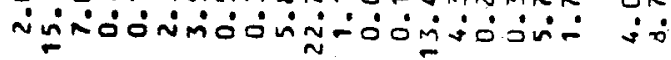

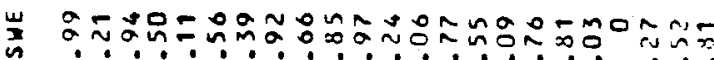

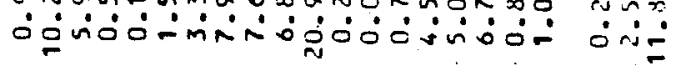

ะ

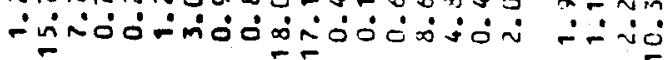

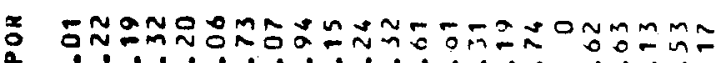
-

z moำ

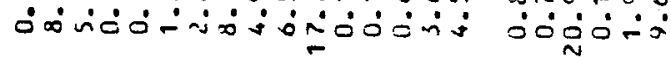

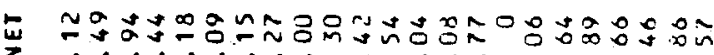
向过-

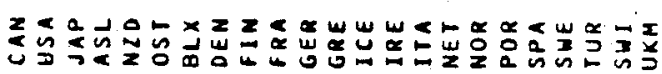




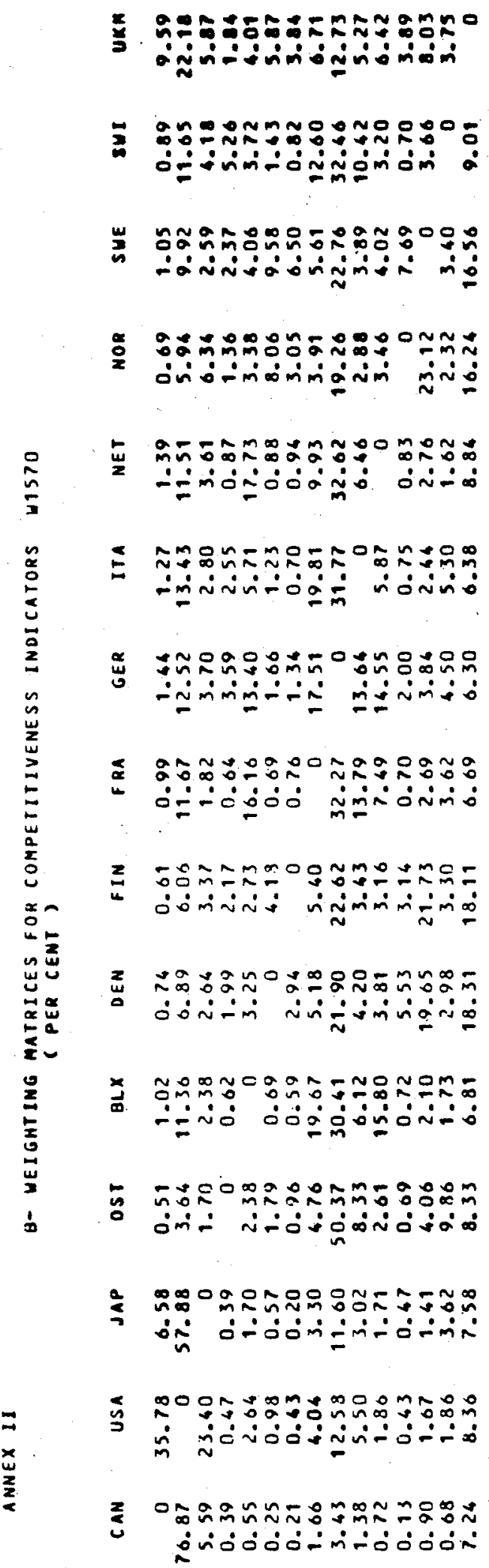

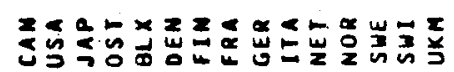

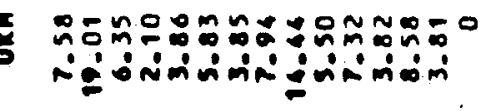

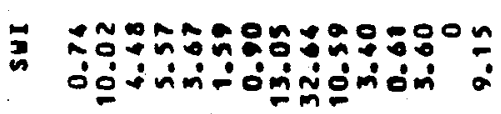

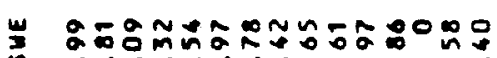

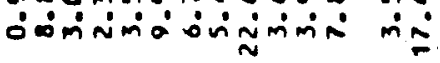

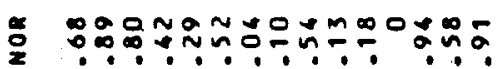

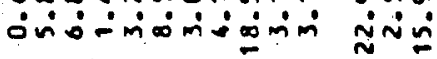

מ.

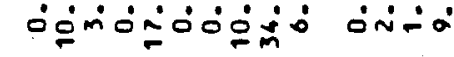

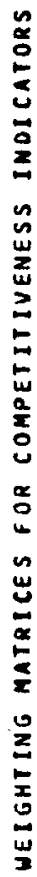

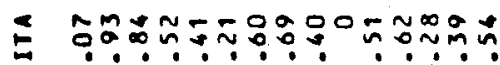

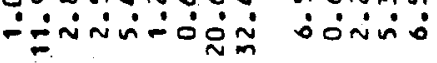

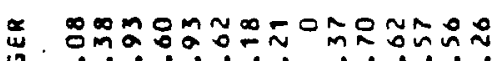

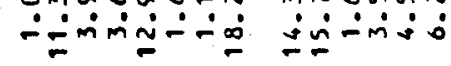

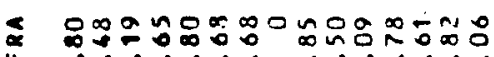
ónidindo

Z

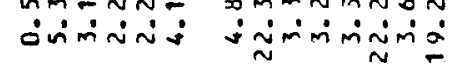

z

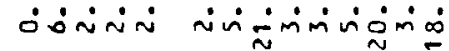

×

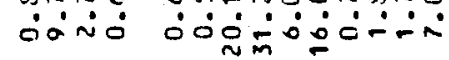

-

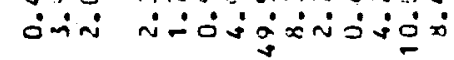

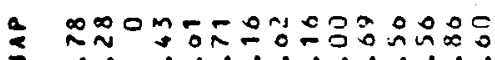

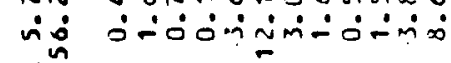

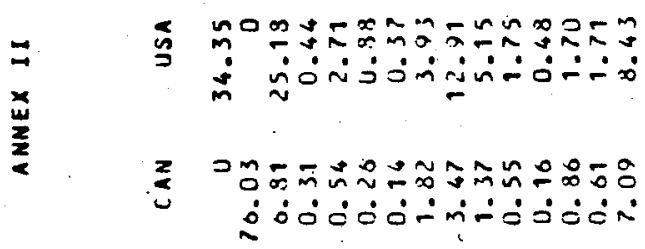

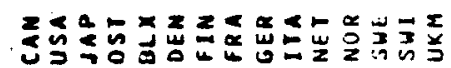




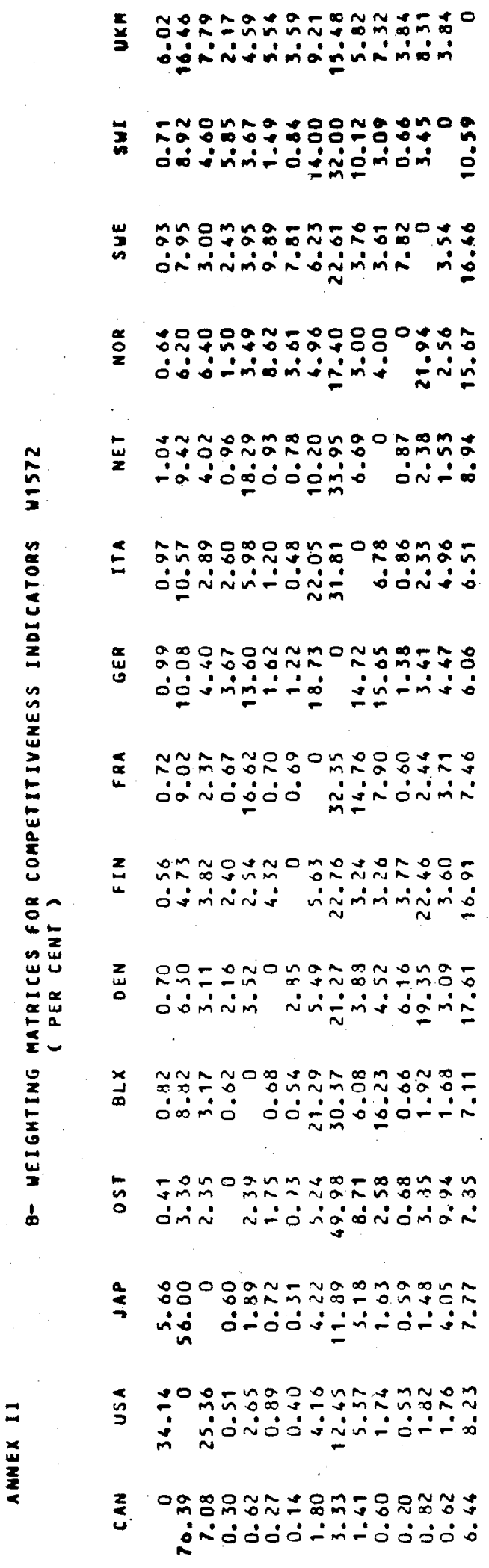

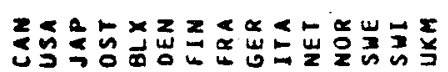

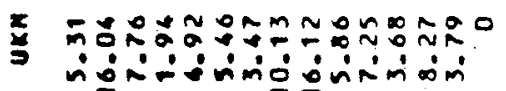

萡

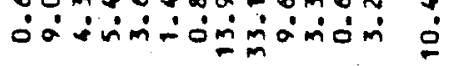

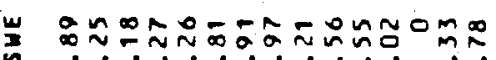

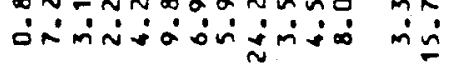

\&

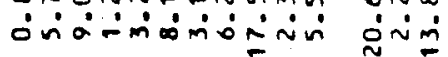

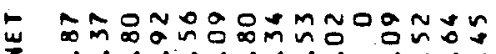

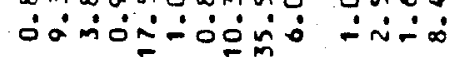

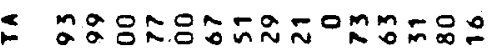

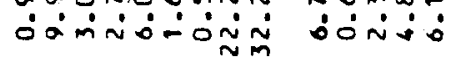

జ

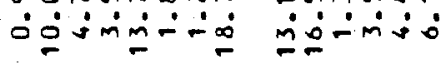

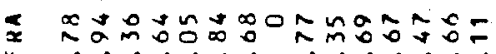

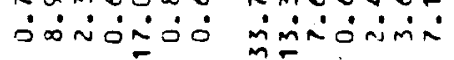

z

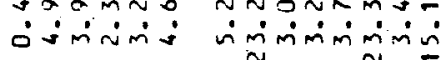

:

恣

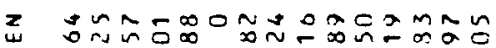

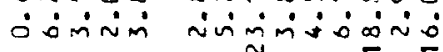

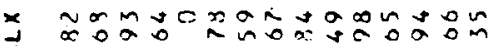

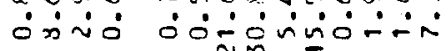

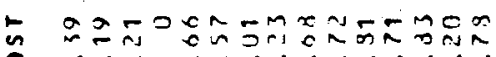

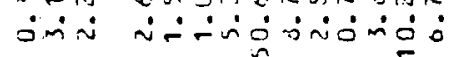

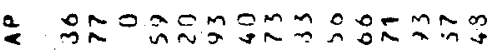

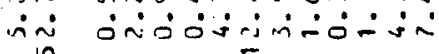

๓

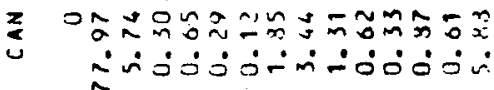

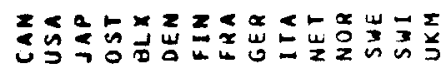




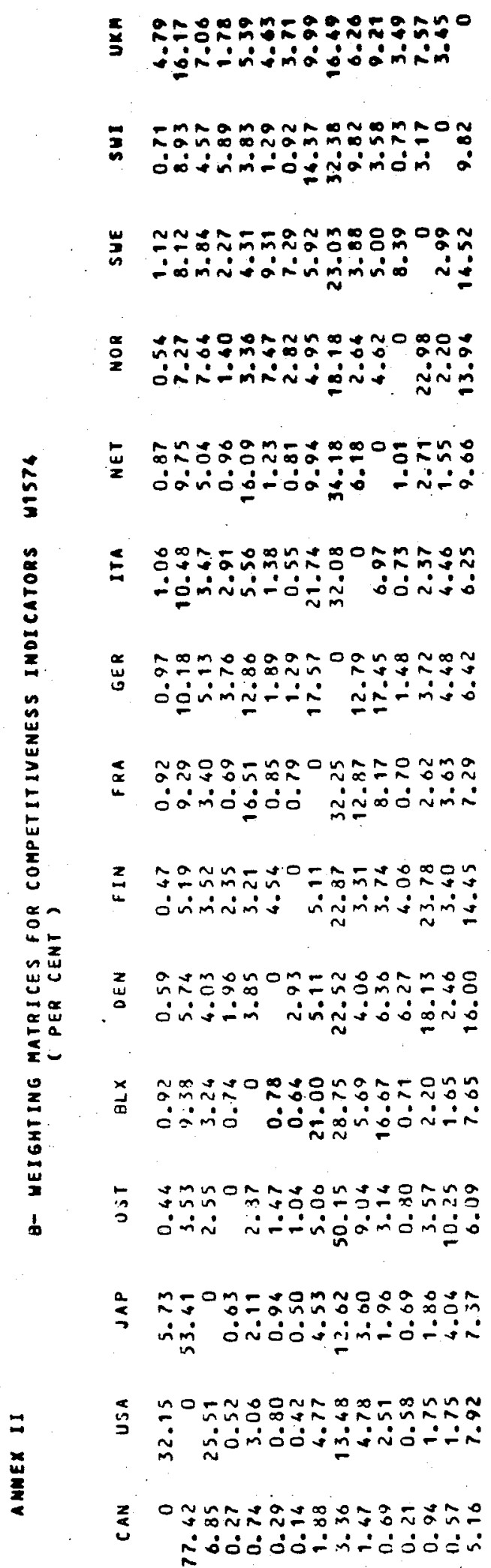

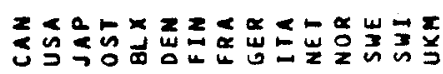
F

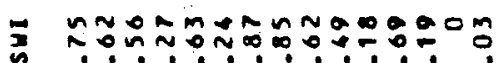

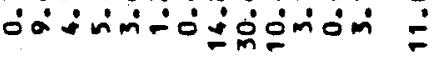

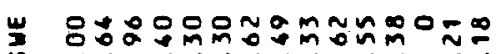

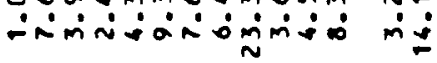

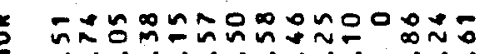

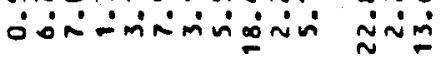

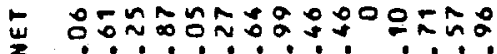

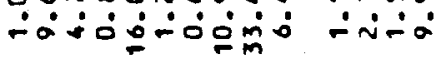

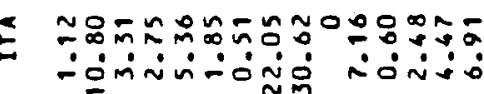

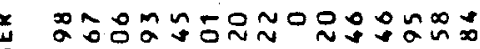

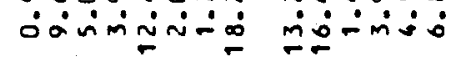

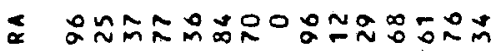

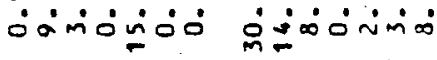

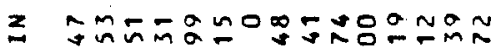

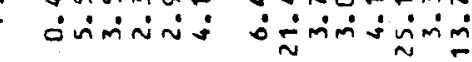

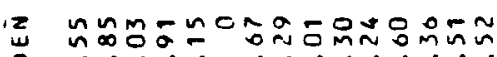

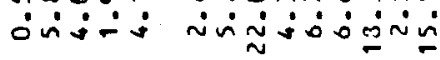

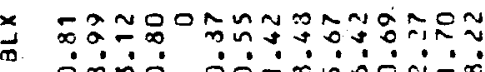

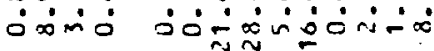

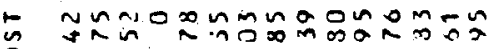
onin r.--nigainérion

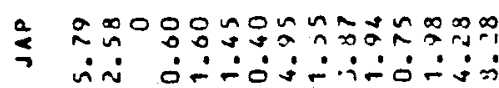

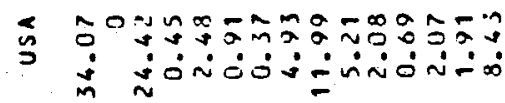

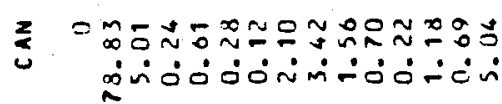

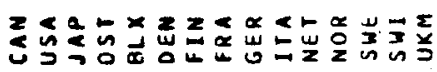




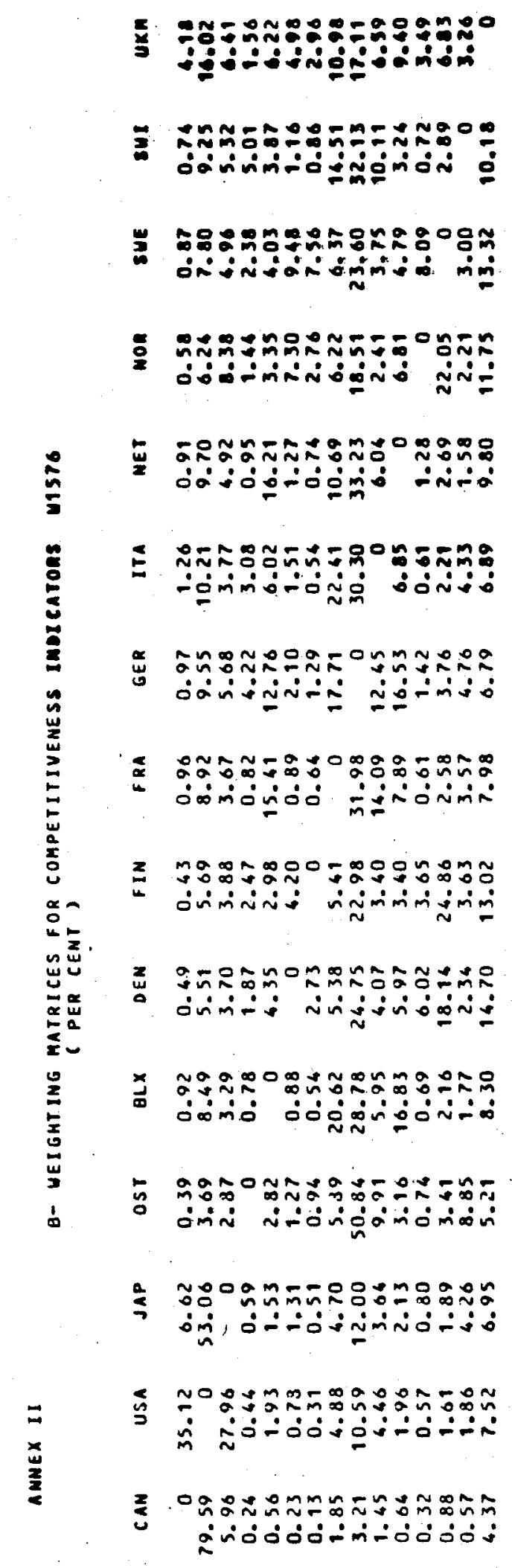

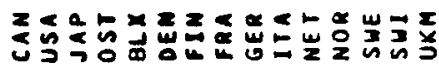

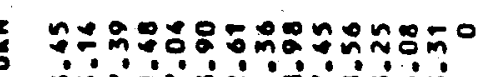

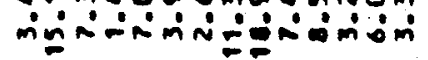

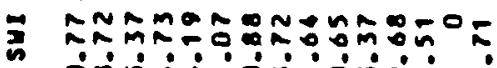

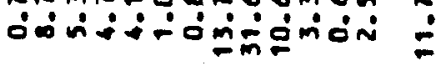

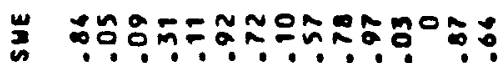

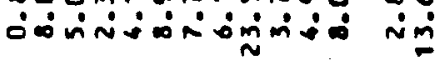

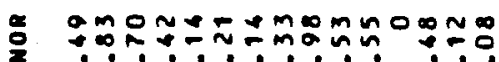

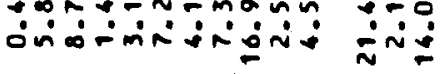

$\hat{n}$

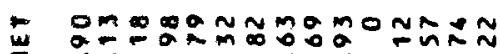

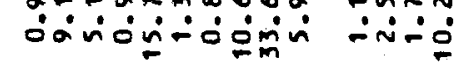

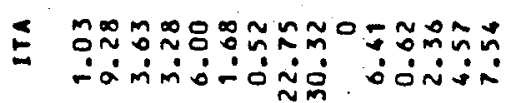

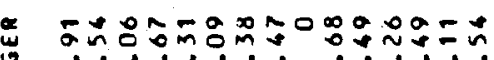

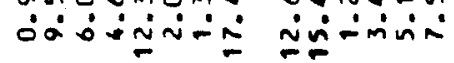

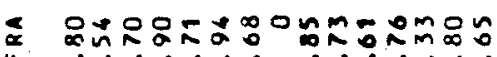
ómí丶0ं

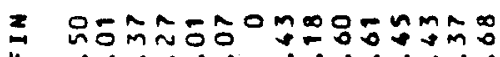

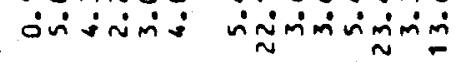

Z onis: $\quad$ inimidisid

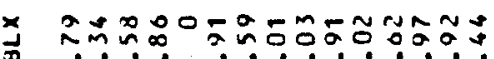
ox

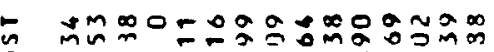

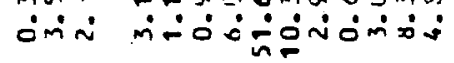

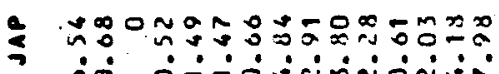

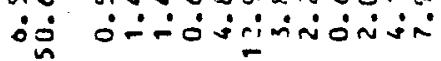

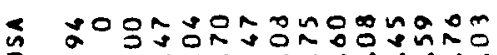

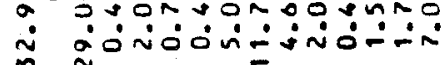

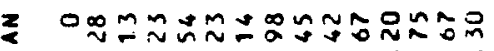

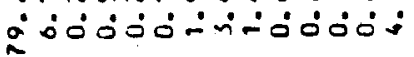




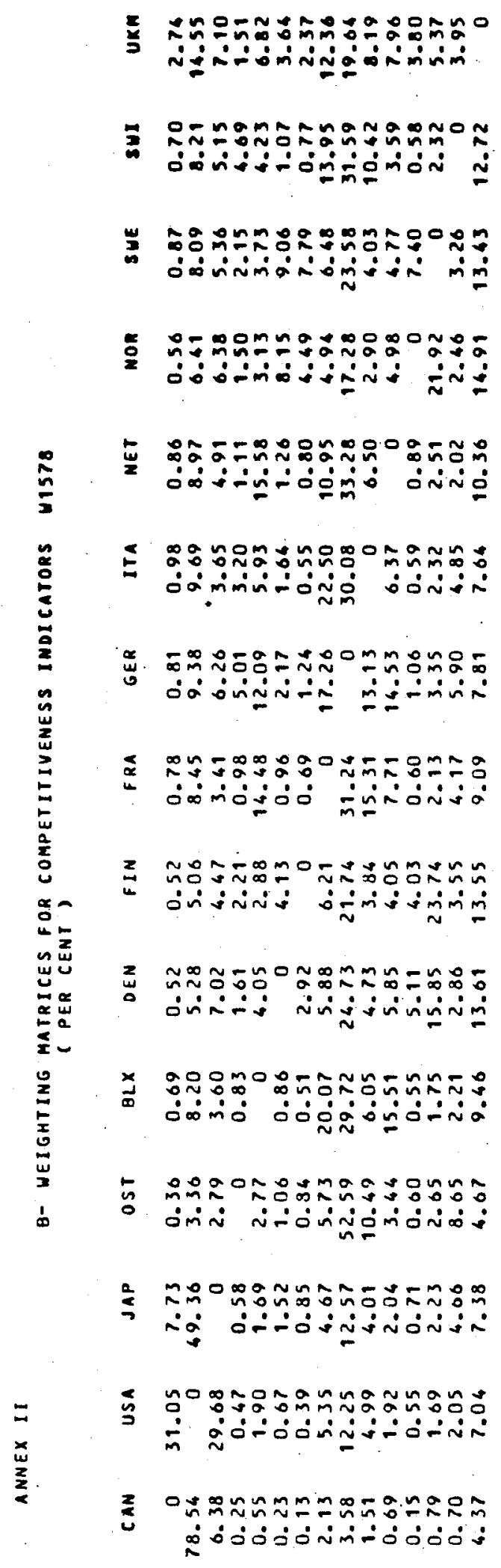

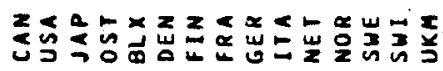

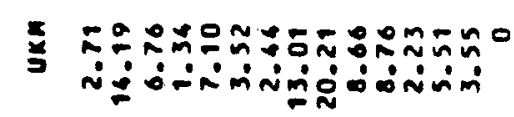

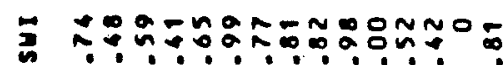

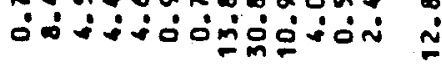

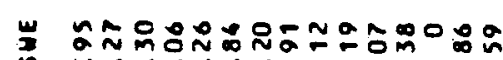

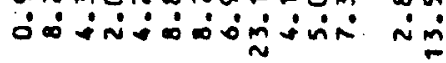

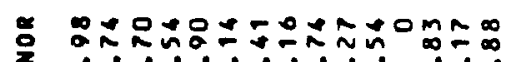

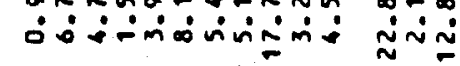

ะ

$\stackrel{a}{n}$

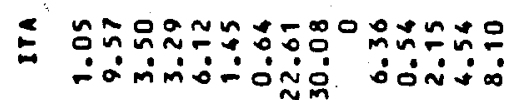

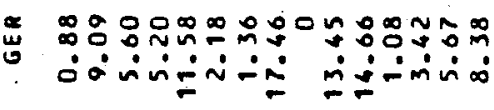

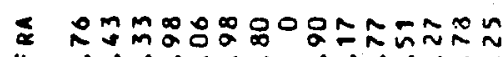

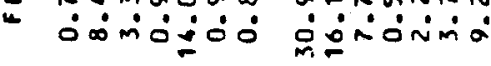

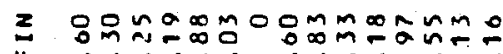

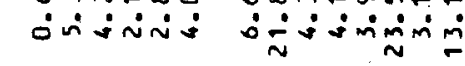

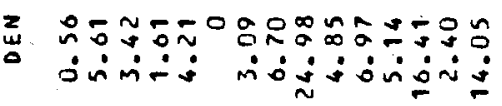

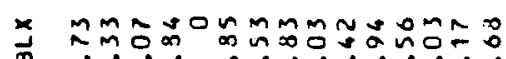

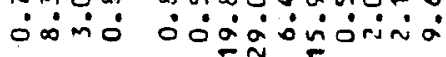

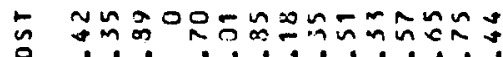

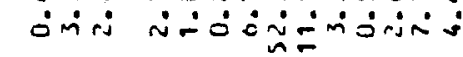

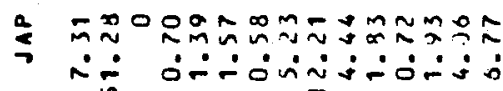

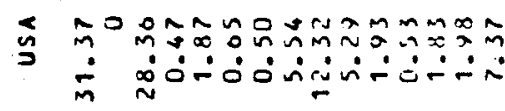

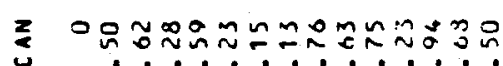

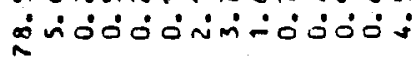

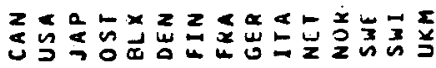




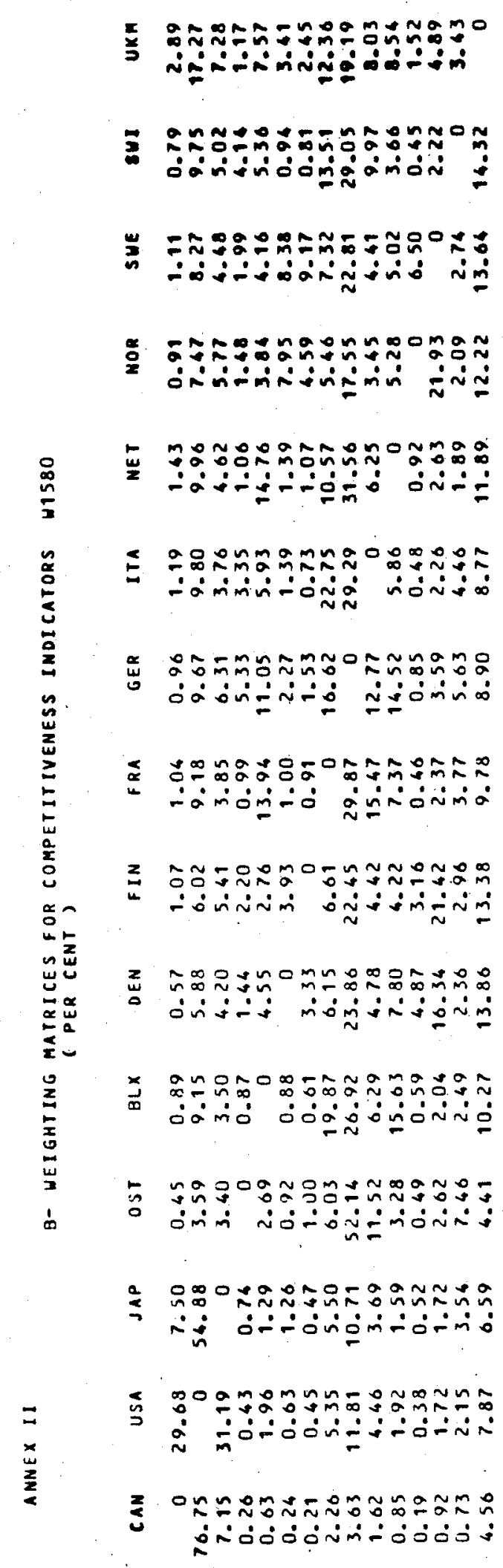

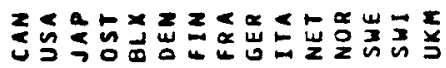

รัดำ

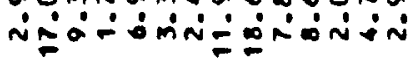

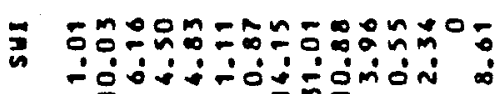

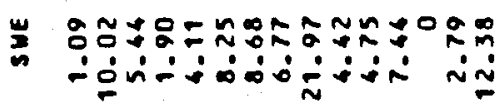

ะ

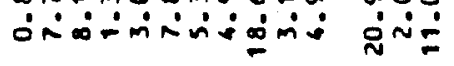

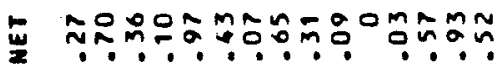

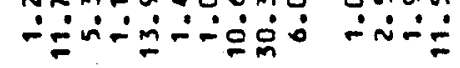

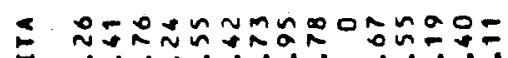

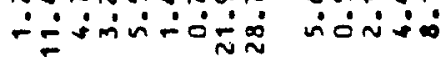

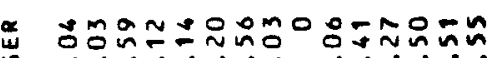

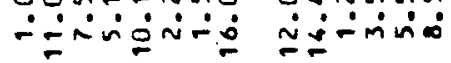

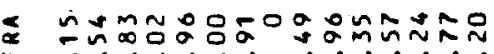

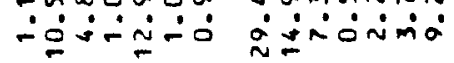

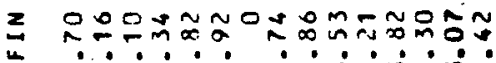

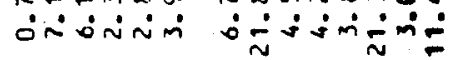

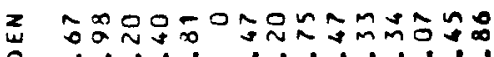

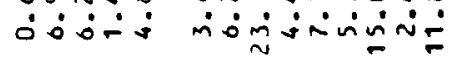

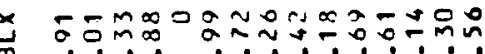

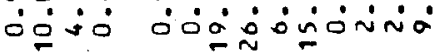

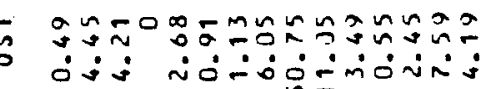

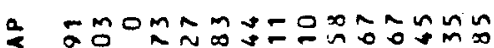

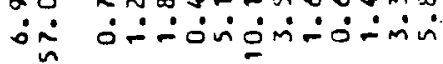

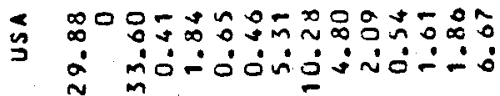

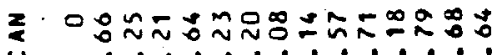

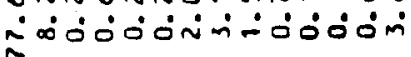

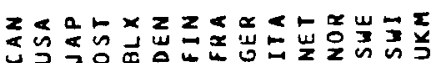




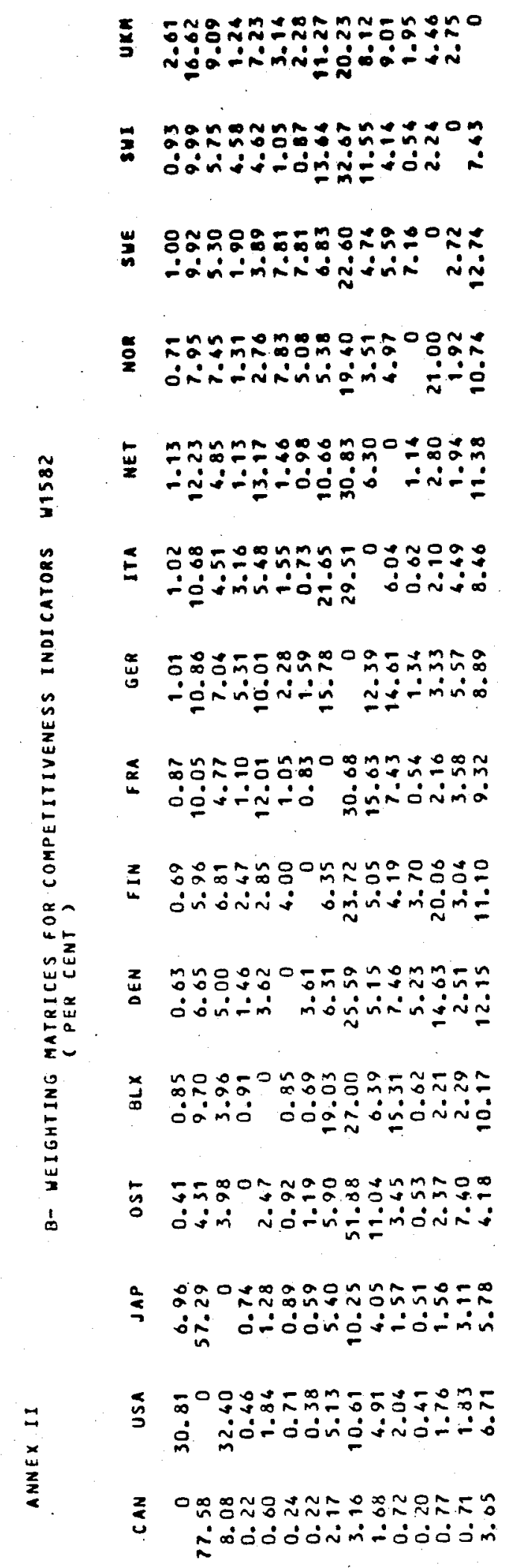

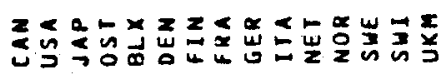

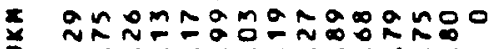

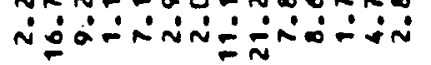

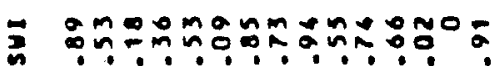

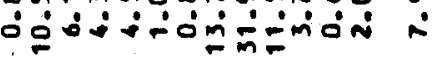

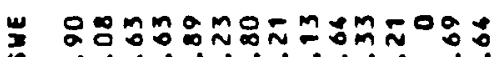

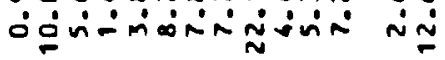

잉

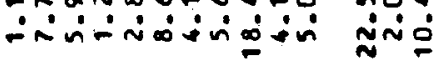

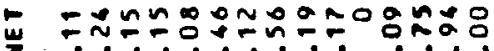

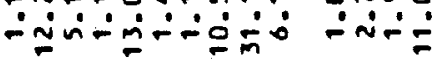

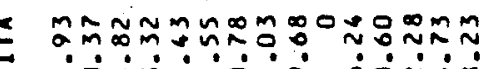

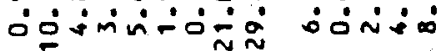

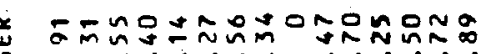

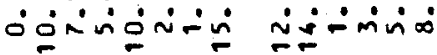

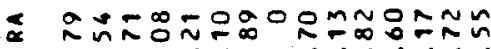

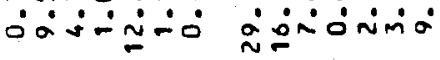

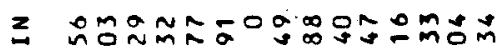

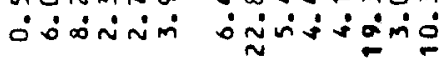

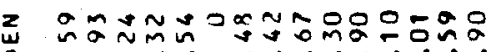

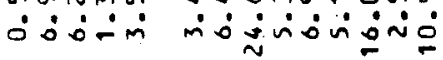

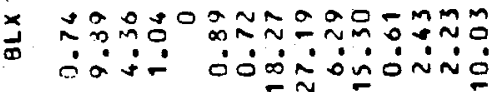

-

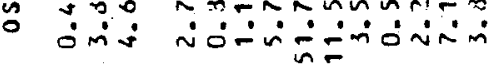

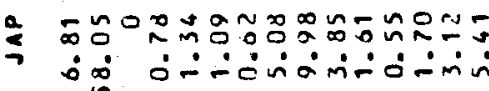

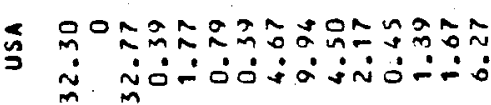

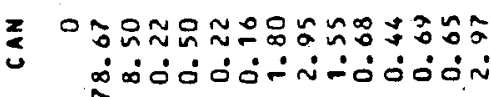

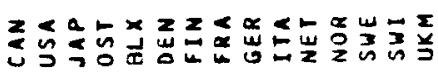




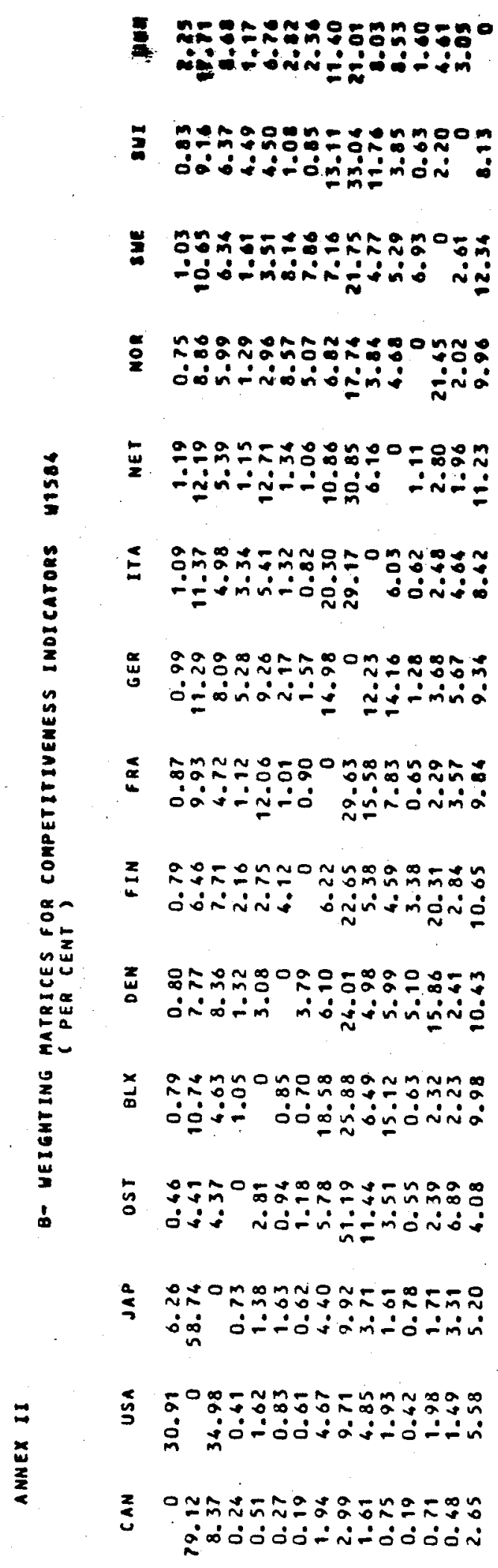

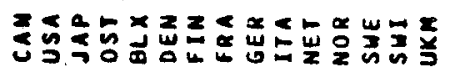


- $39-$

ANNEX 3 


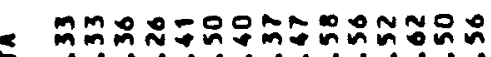

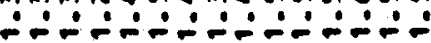

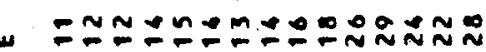

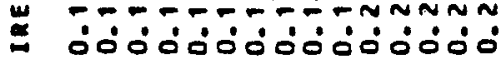

岕

$00000000 \overline{0} \overline{0}$

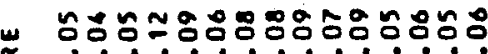

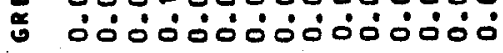

ș

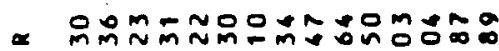

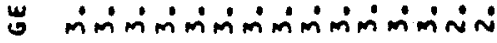

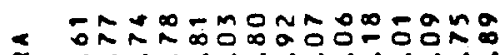

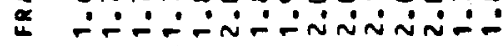

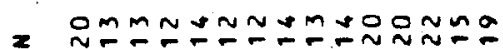

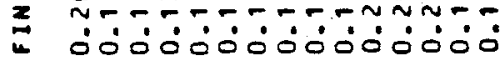

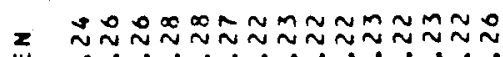

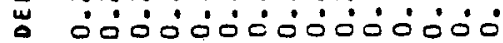

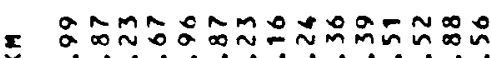

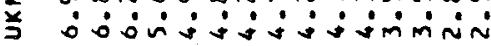

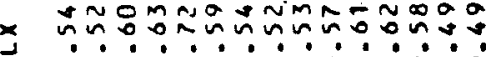

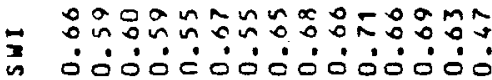

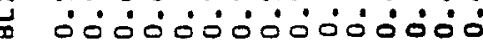

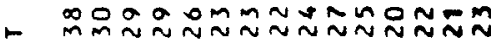

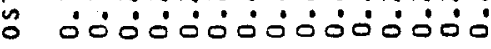

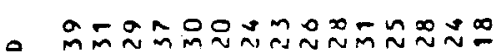

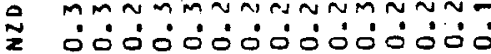

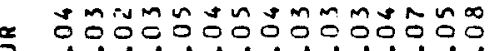

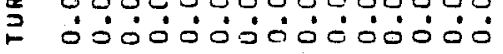

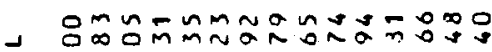

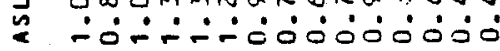

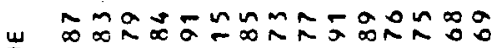

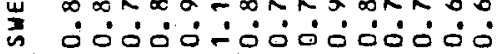

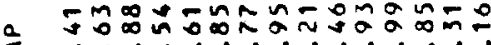

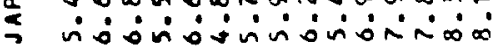

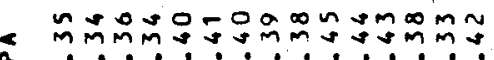

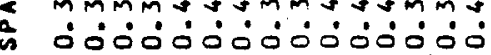

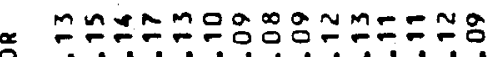

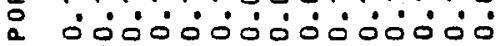

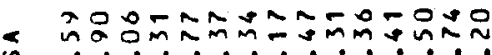

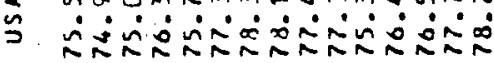

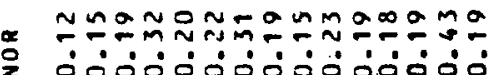

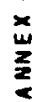

000000000000000

3

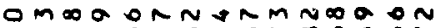

ะ 


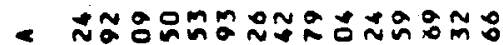

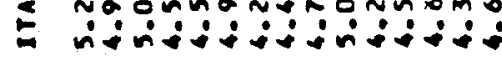

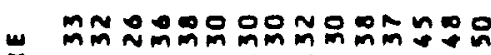

\# dodódododojojód

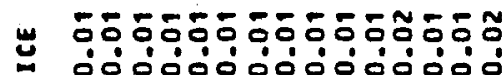

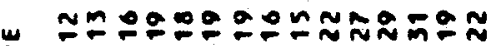

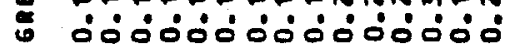

ș

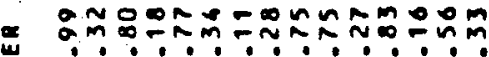

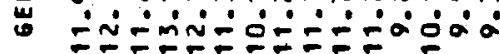

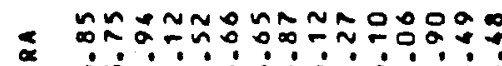

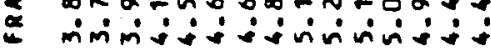

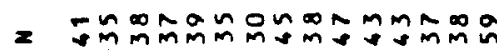

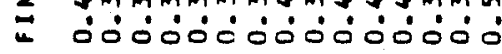

$=$ ดุ

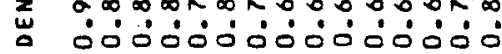

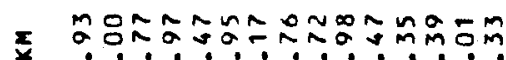

ว

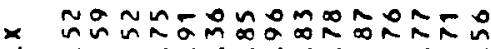

๓

F

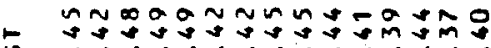

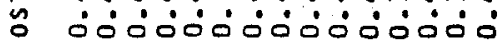

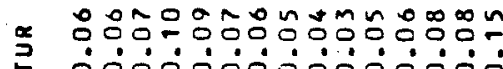

ㅜำ

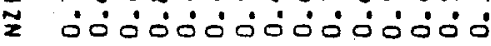

س

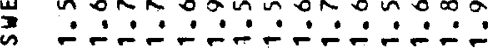

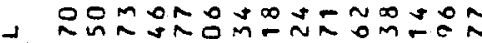

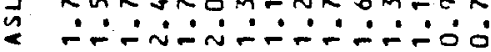

<

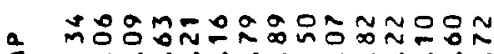

= N

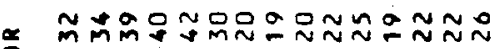

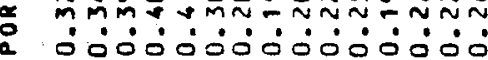

$=$

$=000000000000000$

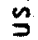

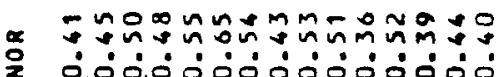

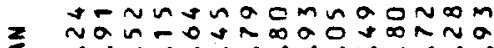

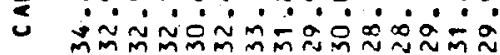

ธ

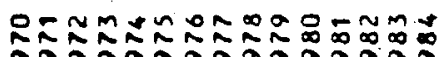

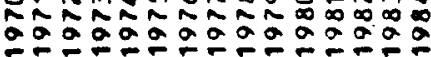




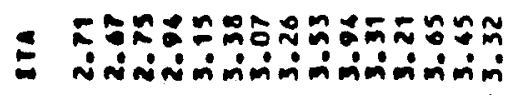

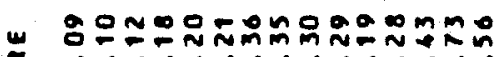

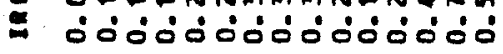

$\underline{w}$

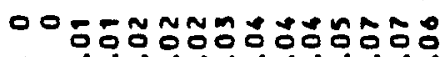

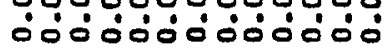

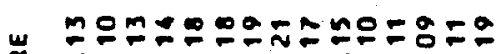

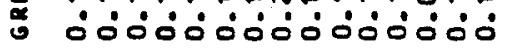

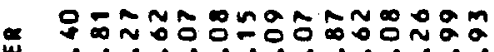

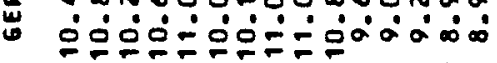

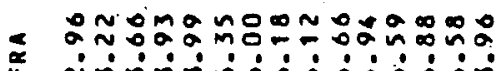

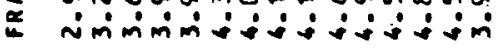

x

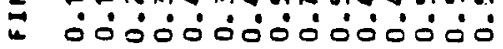

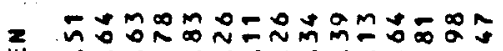

o

,

× กิง

ว

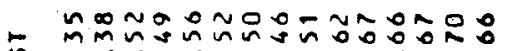

-

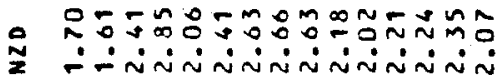

म

«

$\stackrel{a}{2}$

000000000000000

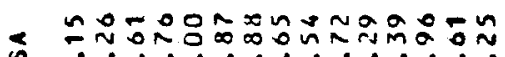

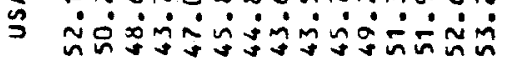

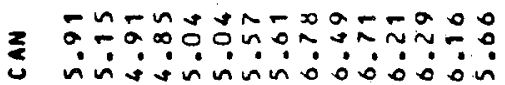

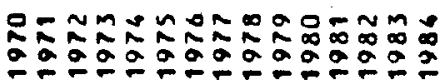

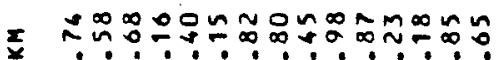

J

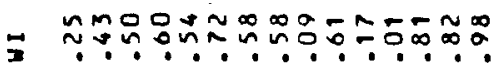

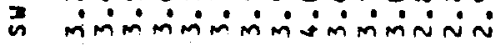

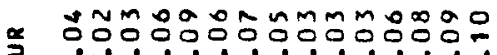

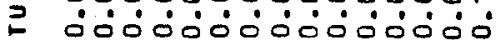

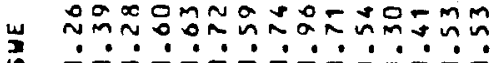

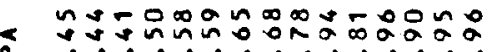

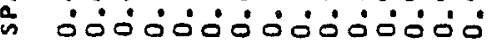

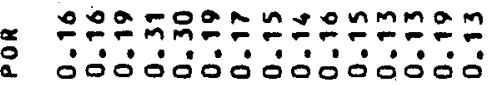

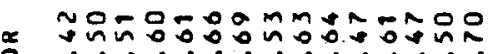

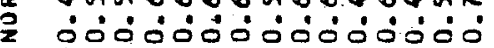

ถนก๊

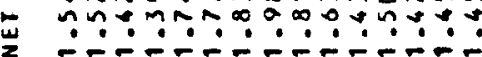

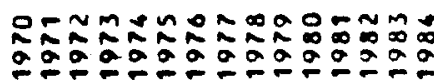




\section{E

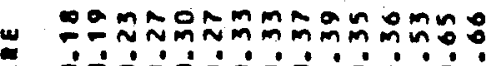 \\ - oódódódódojó.}

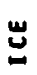

$000 \overline{0}=0.5000000$
000 00

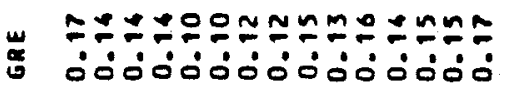

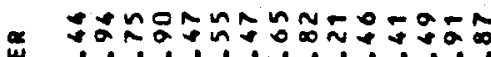

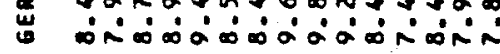

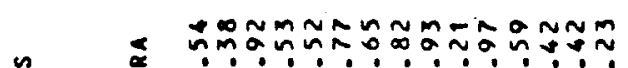

\&

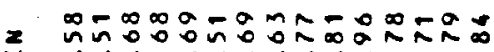

* oهंódódódó

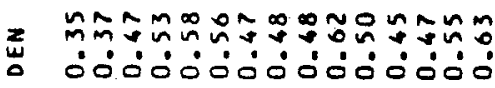

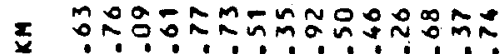

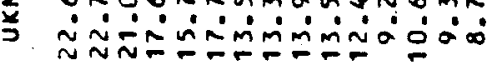

×

ه

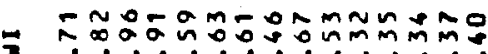

ล

-

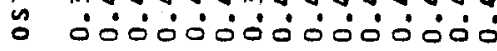

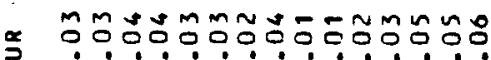

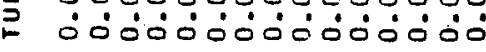

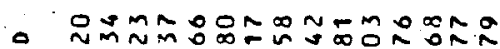

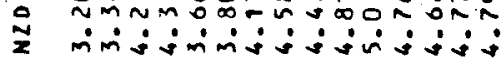

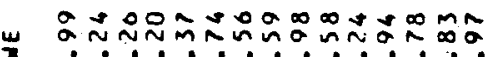

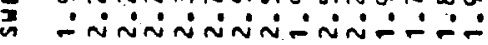

$\vec{n}$

000000000000000

a

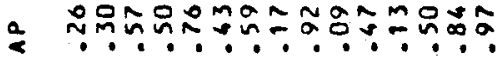

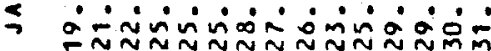

\&

$\Xi$

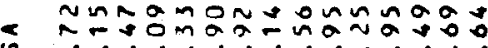

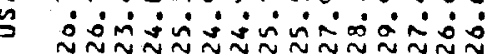

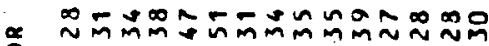

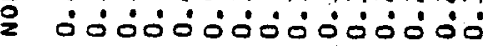

Z

Z

ะ 


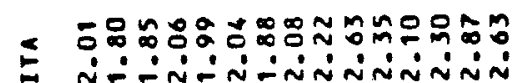

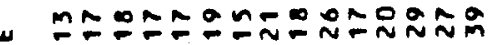

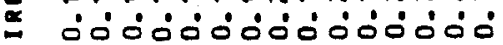

0
$\vdots$
$\vdots$

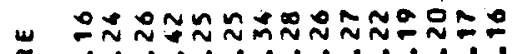

هั

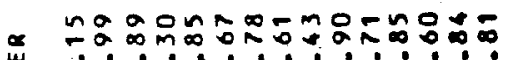

岁

< ก๊ำ

\& $\therefore$ -

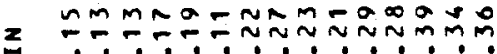

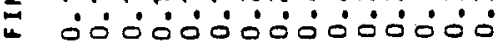

z

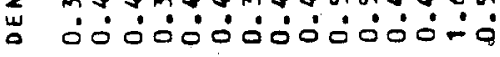

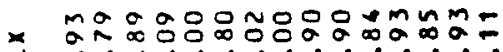

节

紊

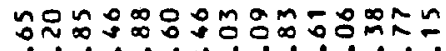

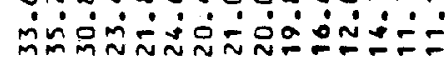

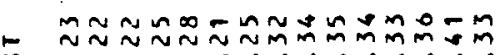

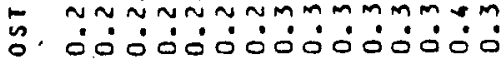

$\frac{e}{2}$

000000000000000

ล

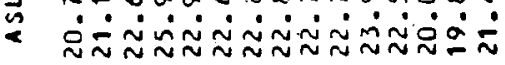

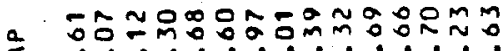

-

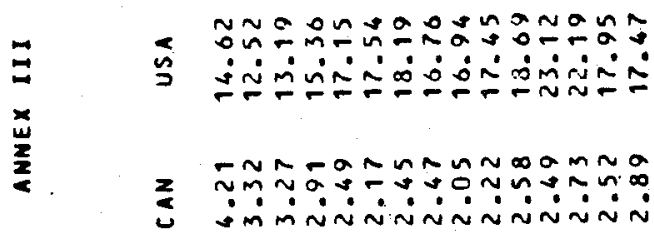

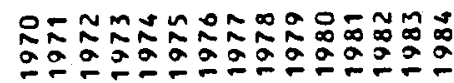

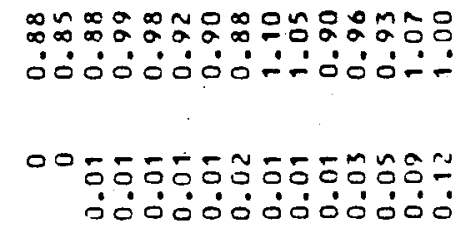

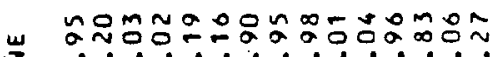

क

a

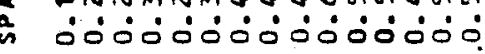

a

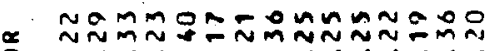

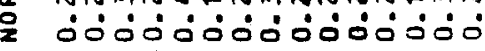

$=\sim 00$ on

E

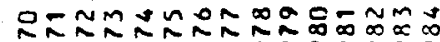

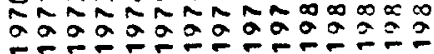




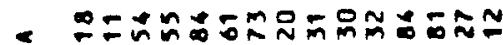 \\ - 0 -}

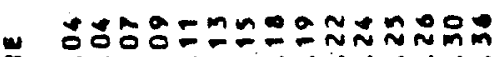

* Sódóóóóódó

"

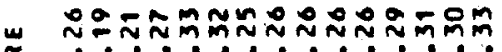

* ododódódodódó

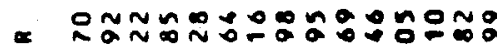

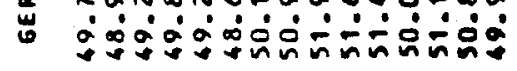

a

<

\&

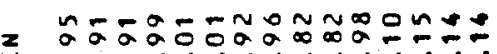

4 0000100000010

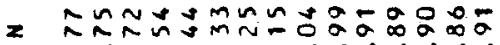

แ

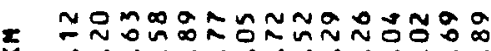

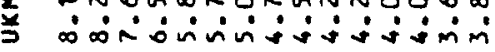

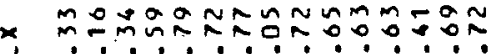

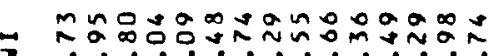

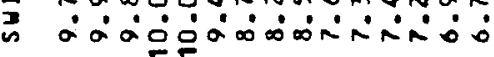

ڤே

000000000000000

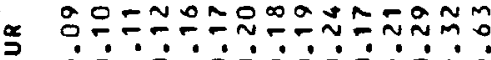

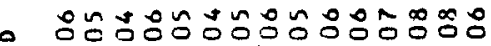

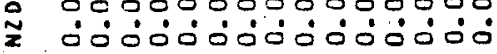

- Oó000000000000

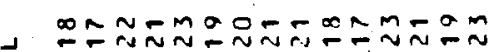

a 00000000000000

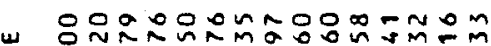

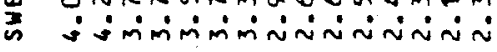

a

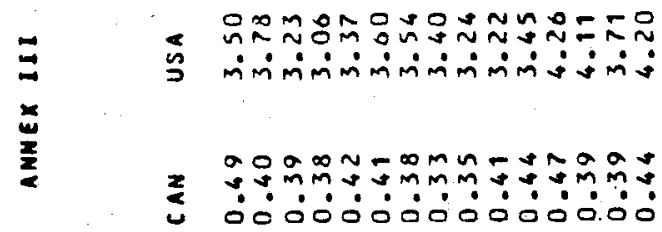

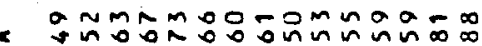

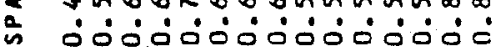

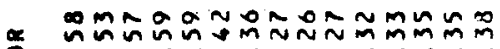

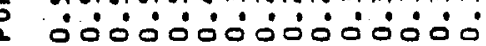

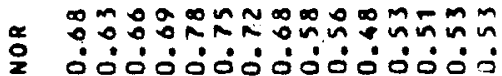

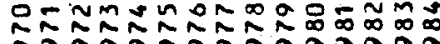

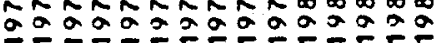

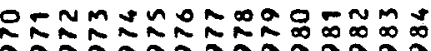

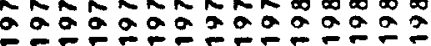




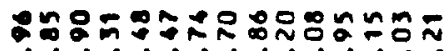

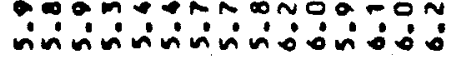

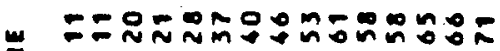

* dodododódodód

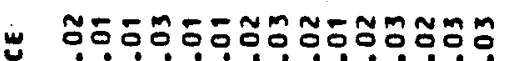

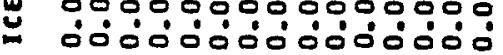

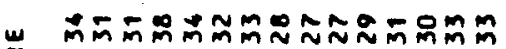

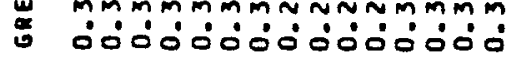

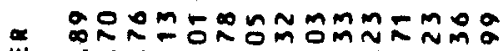

แ

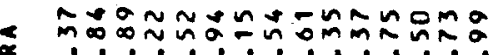

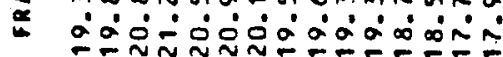

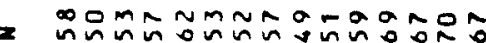

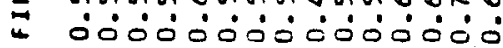

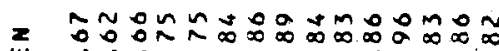

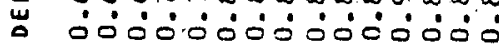

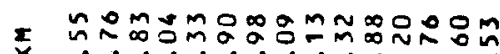

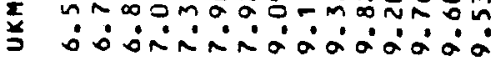

$\times 000000000000000$

इ

$\vec{\Phi}$

-

ㅇำ

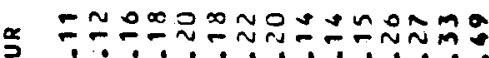

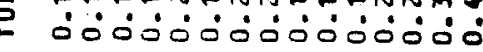

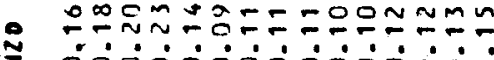

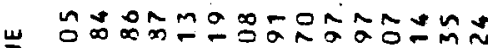

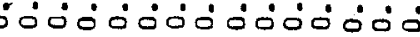

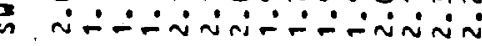

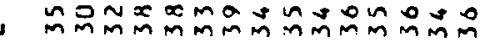

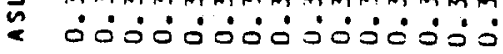

< ํำ

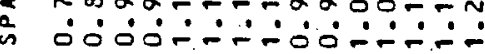

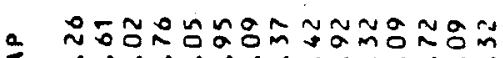

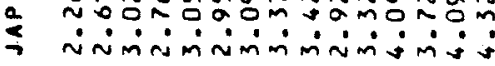

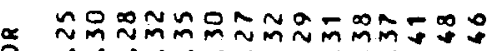

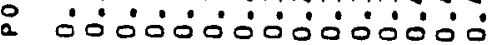

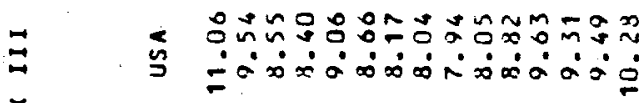

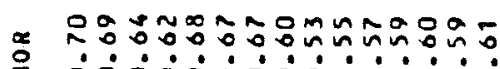

$\underset{2}{\frac{x}{2}}$

z

odododódojojo.

$0000000=000000$

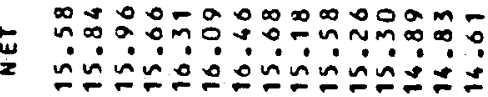

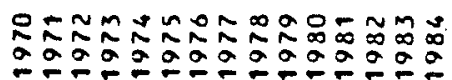

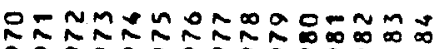

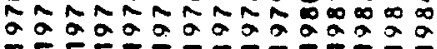




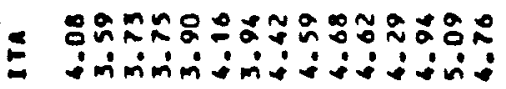

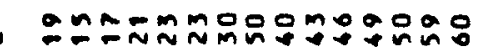

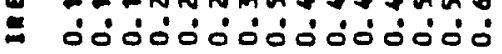

"

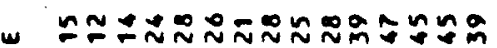

* ódódódododó

\&

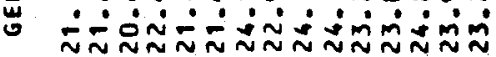

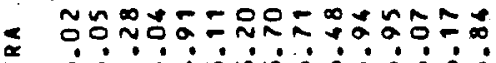

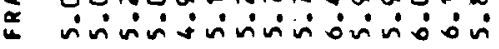

$=\infty_{\infty}^{\infty}: \infty n-\infty$

- ivinivivisimmonmmin

$=000000000000000$

蛋

ร

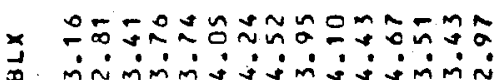

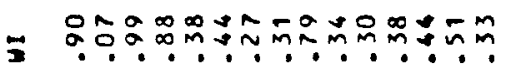

a iminininivinininin

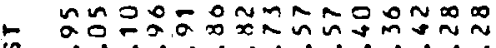

ต

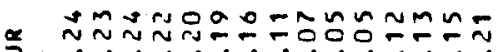

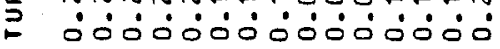

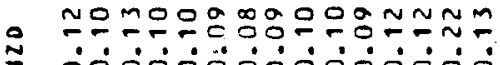

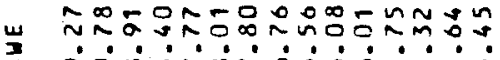

a

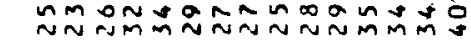

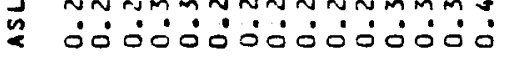

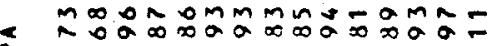

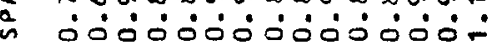

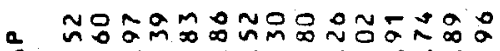

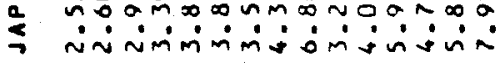

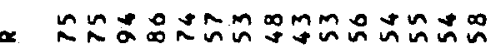

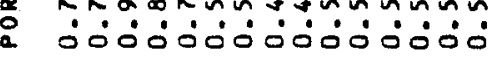

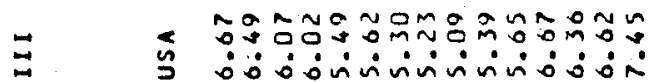

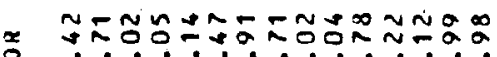

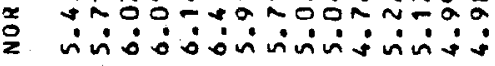

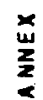

z

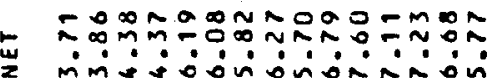




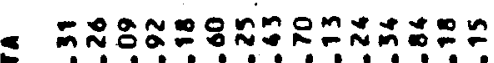 minininiminimititifiñ}

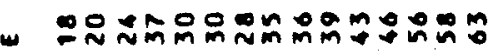

* Sodododódódod

乡 món

แ จกำ

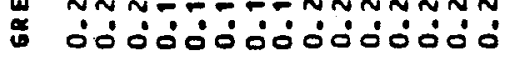

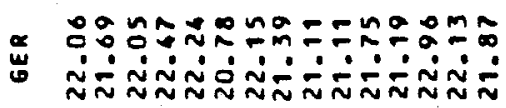

\&

$z$

000000000000000

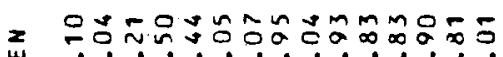

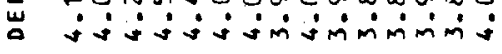

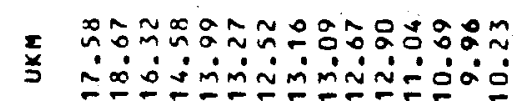

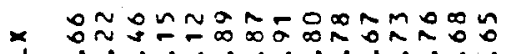

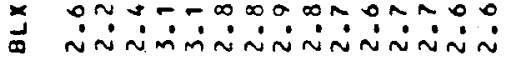

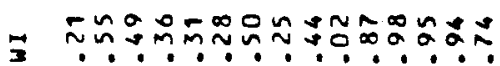

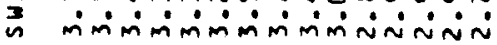

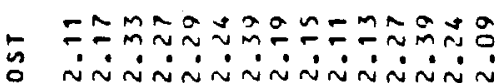

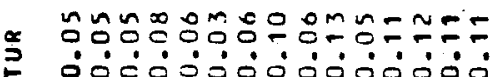

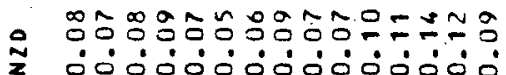

"

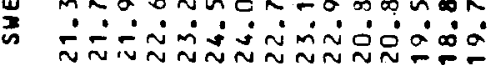

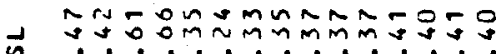

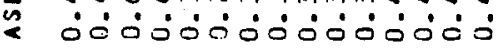

<

文

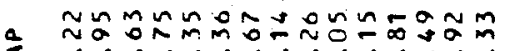

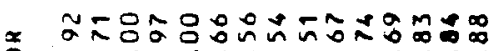

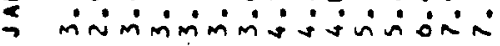

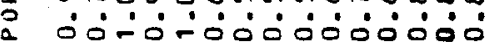

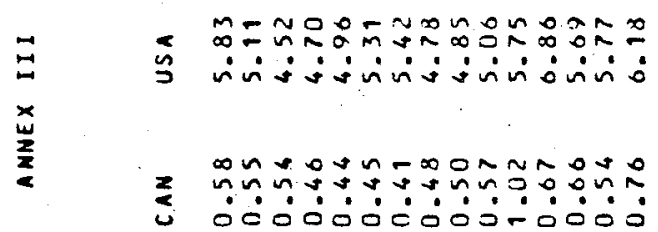

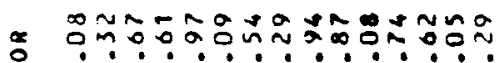

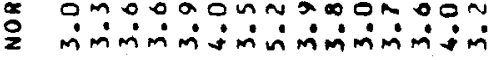

Е

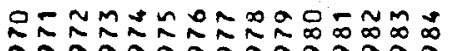

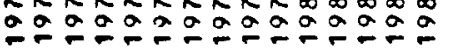




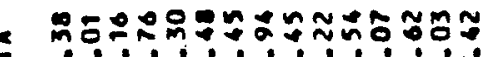

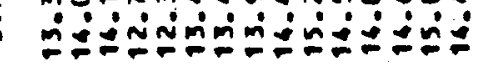

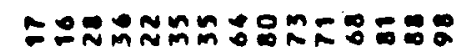

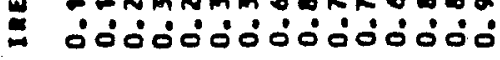

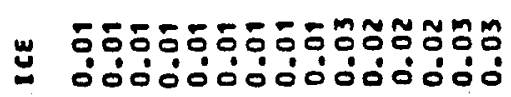

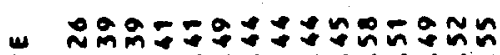

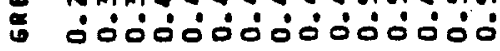

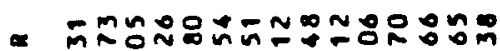

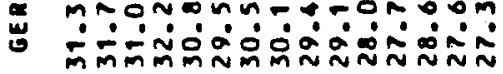

000000000000000

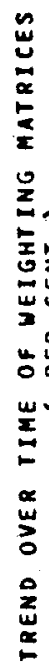

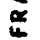

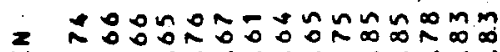

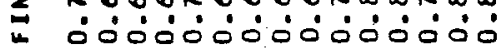

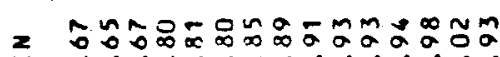

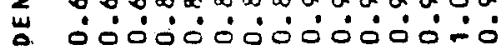

₹

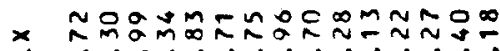

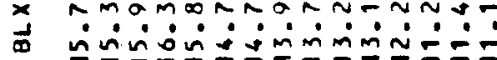

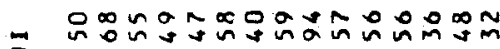

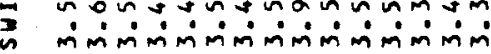

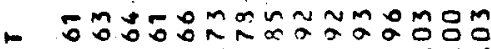

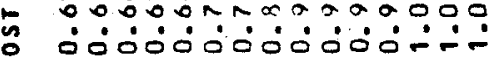

×

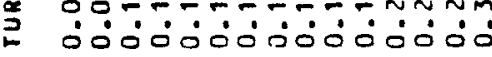

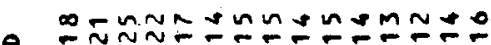

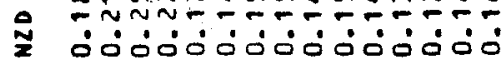

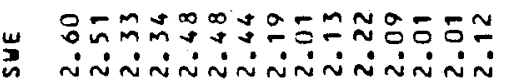

ด

ล

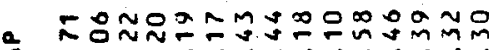

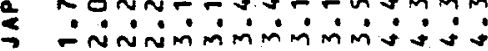

o

\& ojöodódódó

$=$
$\vdots$
$x$
$z$
$z$

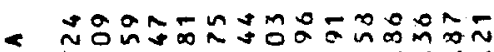

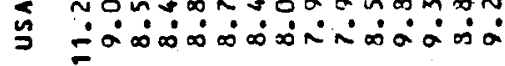

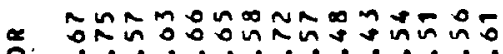

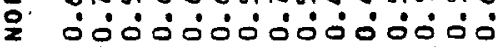

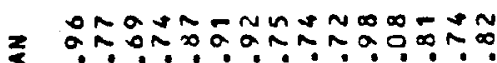

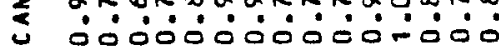

$=\tilde{N}$ m

z

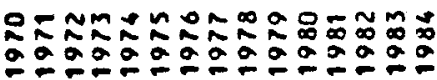


జ

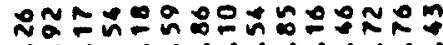

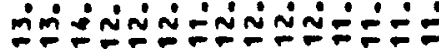

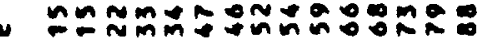

- dóóóóóódóó

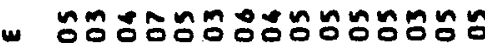

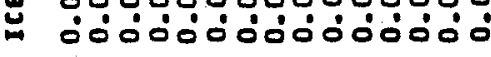

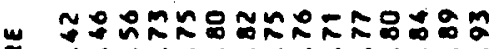

* ó0ंóó

000000000000000

出

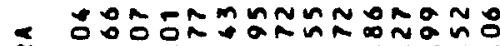

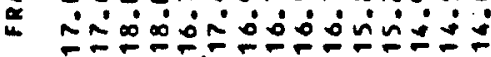

z

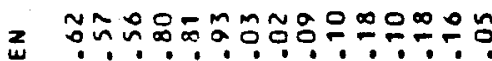

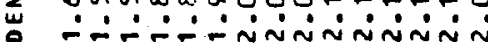

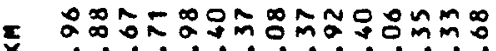

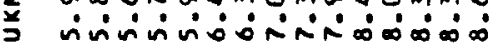

능

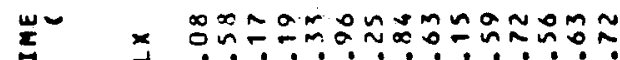

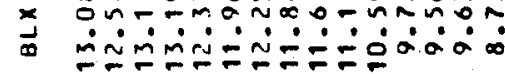

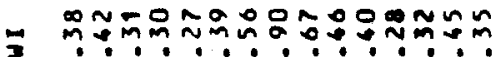

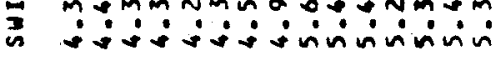

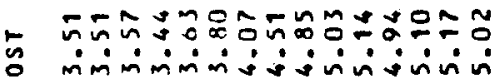

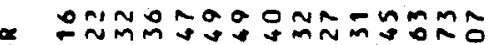

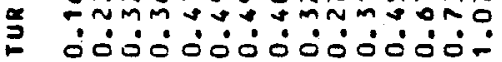

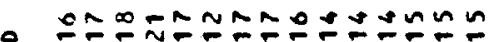

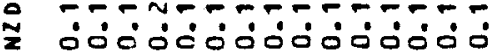

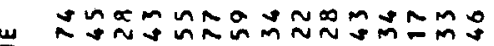

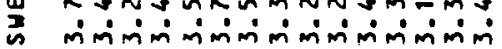

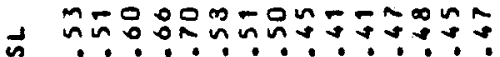

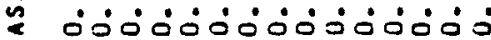

ล กำ

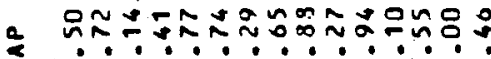

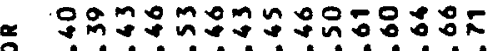

- mimisidivivivivisosis

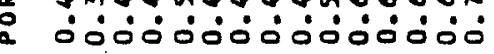

$=$

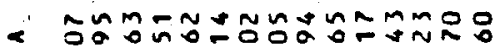

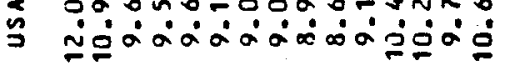

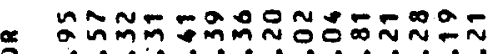

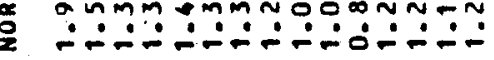

z

3 - 0000000000000

-

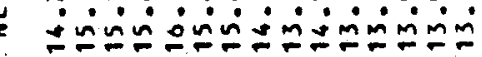

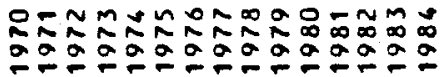

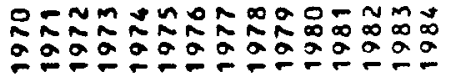




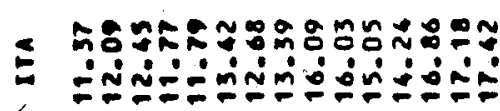

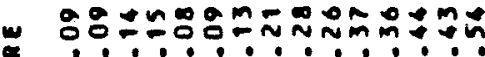

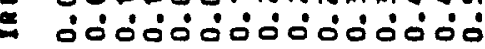

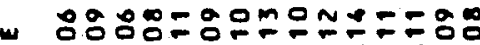

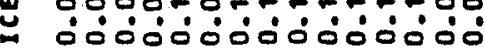

$\underset{*}{*}$

000000000000000

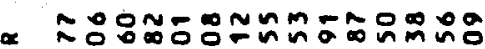

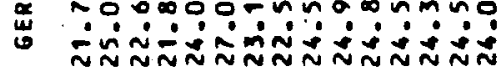

5

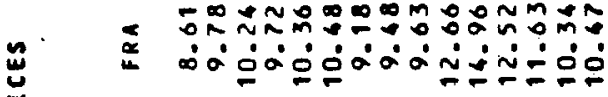

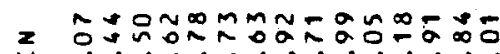

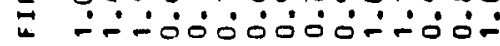

ะ

แ

蕠

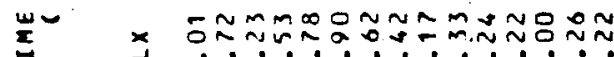

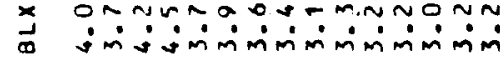

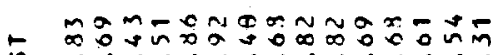

$\Leftrightarrow \because \because \because \because \because \because \because \because \because$

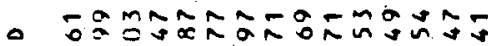

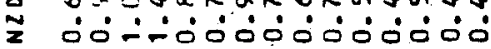

a

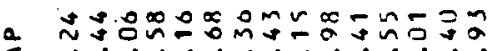

smimping

$\Xi$
$\vdots$
$\frac{4}{2}$
$\frac{\pi}{2}$

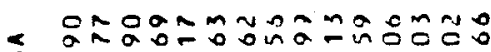

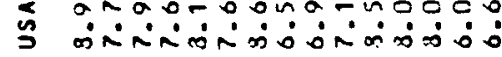

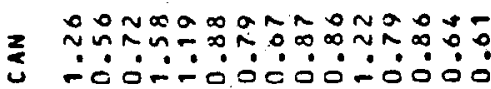

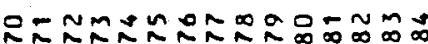

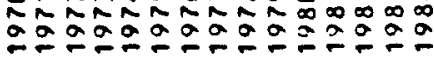

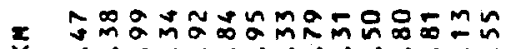

年

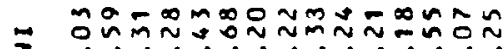

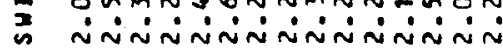

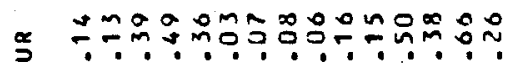

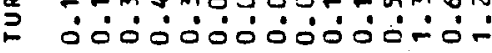

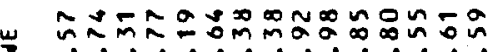

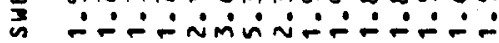

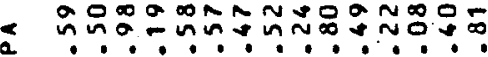

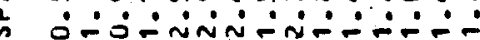

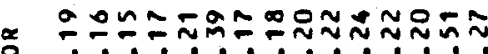

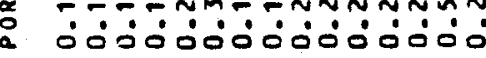

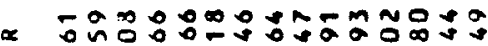

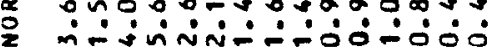

=

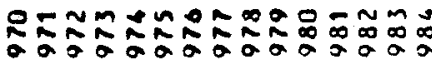




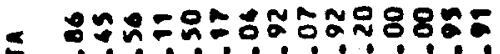

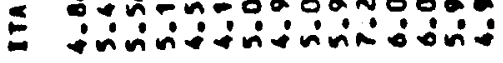

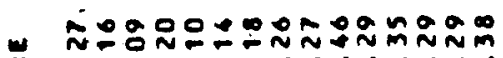

\# dódóódódódó

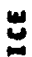

000000000000000

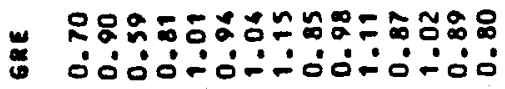

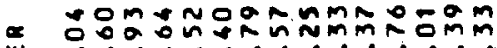

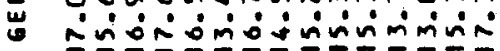

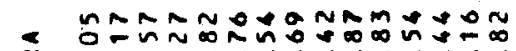

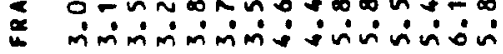

z

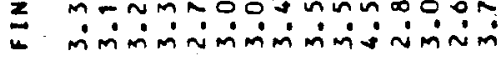

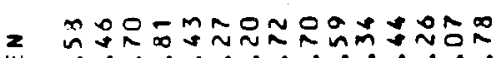

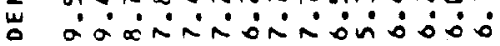

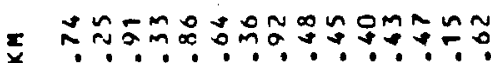

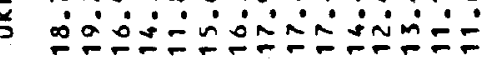

:

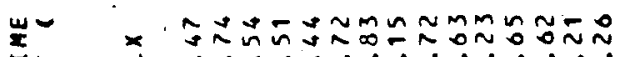

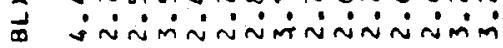

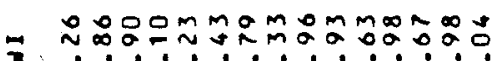

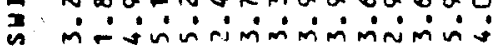

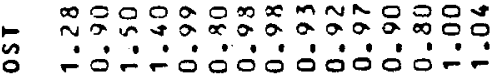

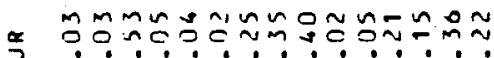

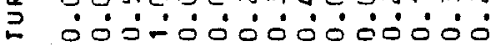

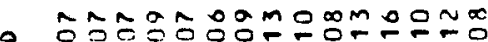

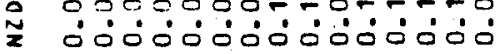

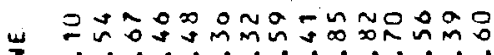

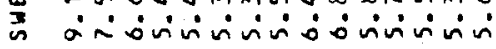

-

a jojomiogojojojoj

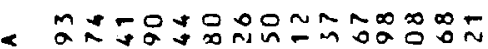

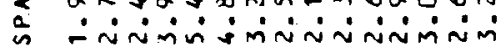

\&

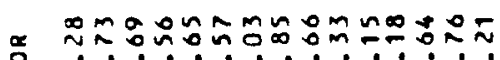

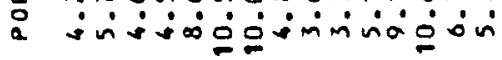

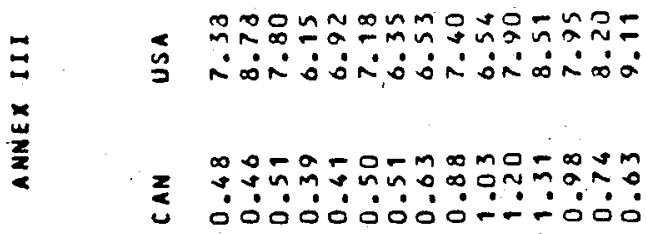

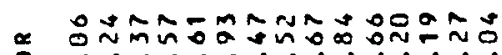

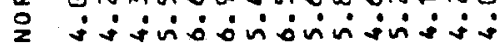

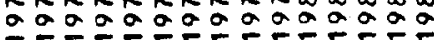




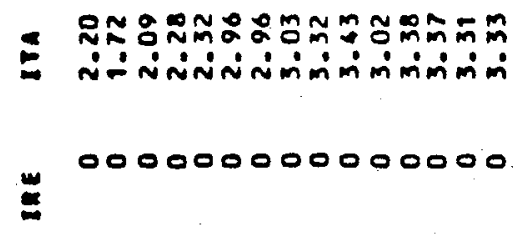

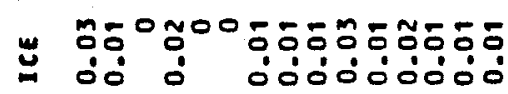

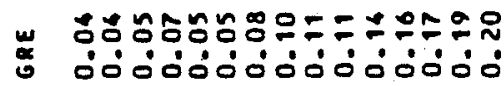

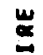

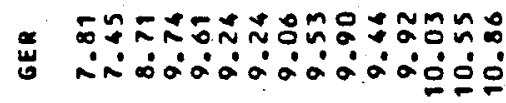

\&

* inimititio:0000

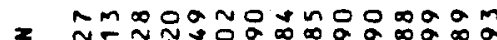

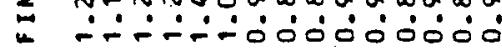

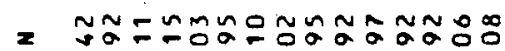

a

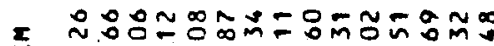

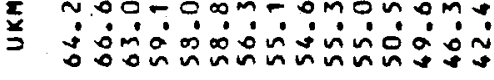

$x$ กีะ

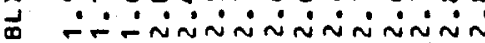

- ํํำํํำㅇำ

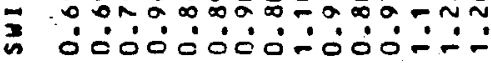

-

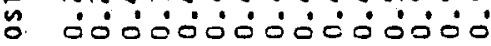

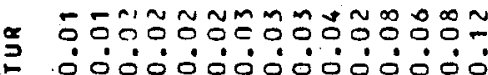

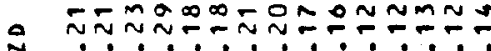

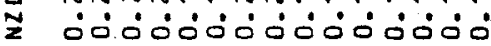

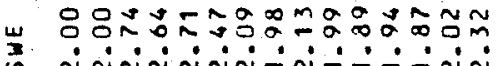

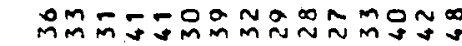

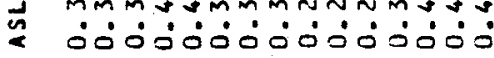

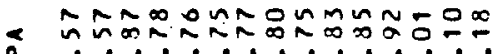

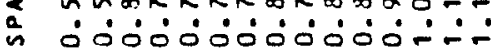

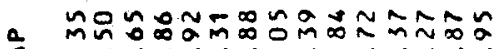

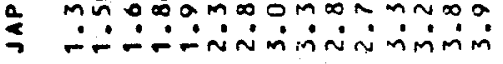

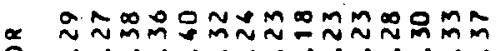

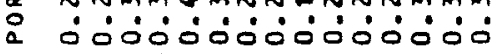

$\equiv$

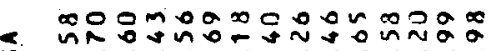

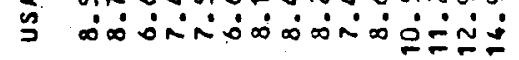

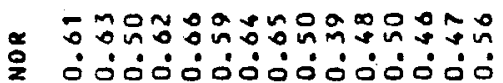

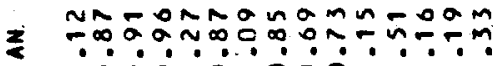

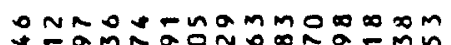

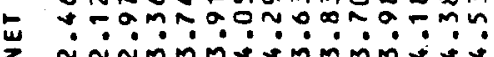

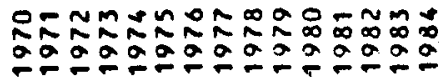

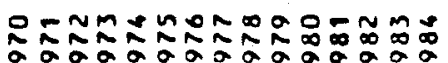

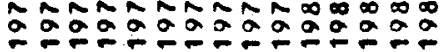


E

000000000000000

«

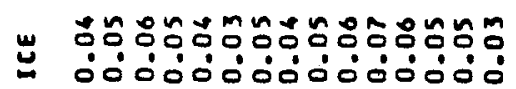

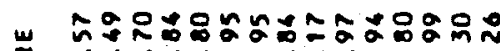

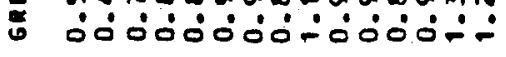

$\vdots$

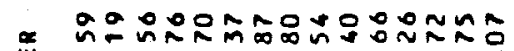

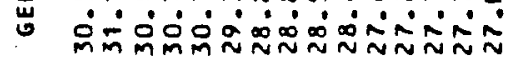

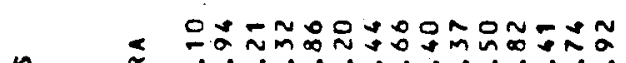

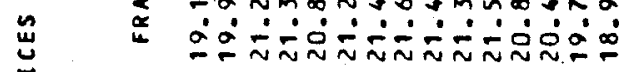

z oñ

z ódodidódidó.

z

×

แ

×

ब

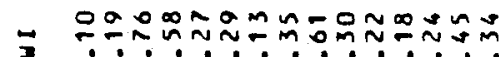

- จง

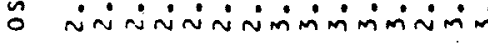

s

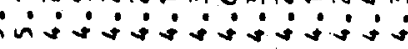

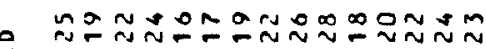

i 000000000000000

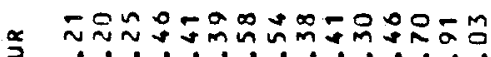

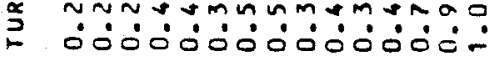

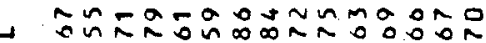

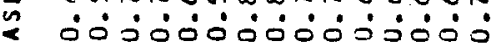

虫

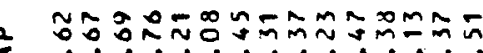

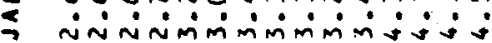

ะ

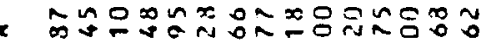

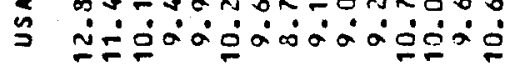

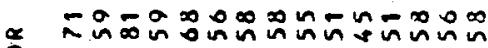

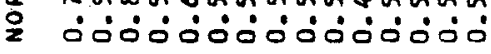

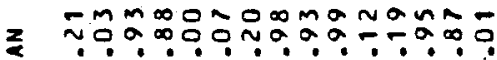

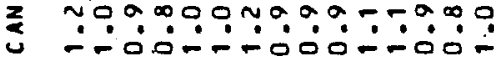

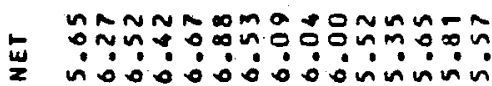




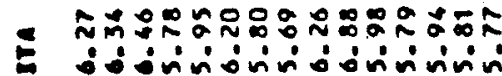

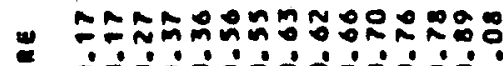

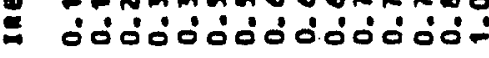

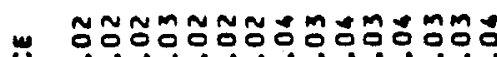

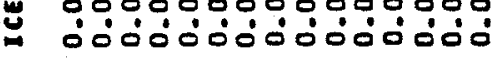

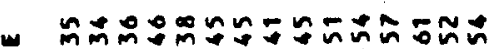

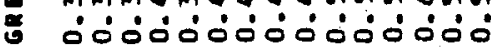

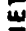

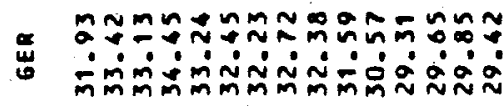

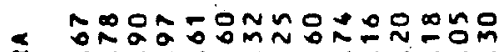

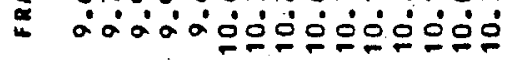

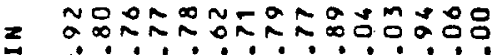

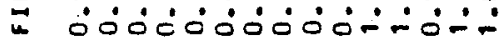

z

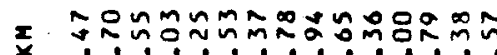

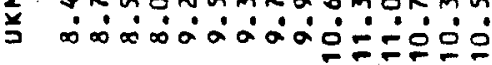

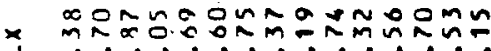

a

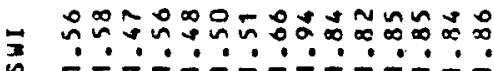

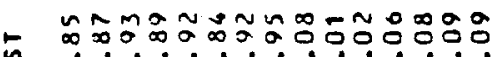

ล

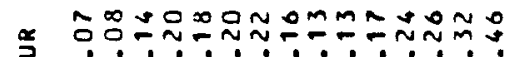

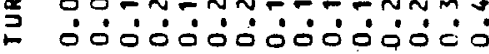

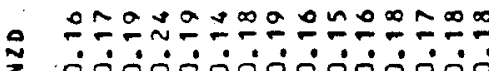

山 :

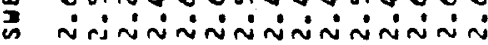

म

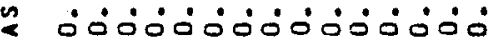

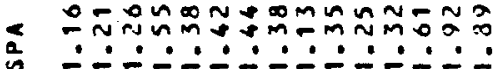

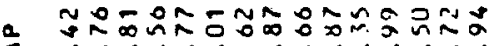

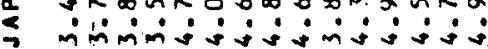

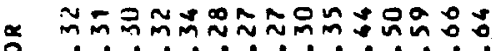

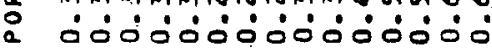

$\equiv$
$\vdots$
$\vdots$
$\frac{2}{2}$
2

\&

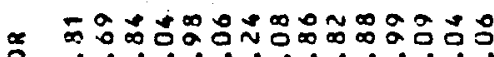

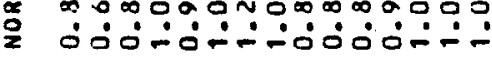

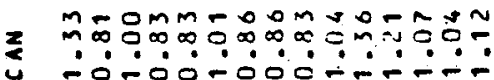

$\stackrel{5}{z}$

000000000000000

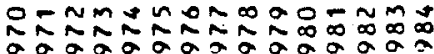

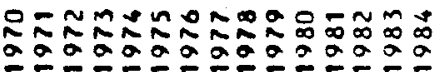




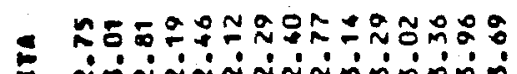

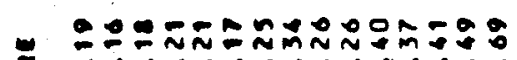

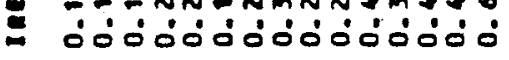

\

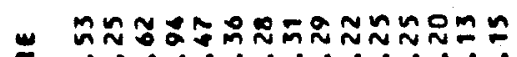

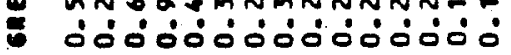

\& 巴

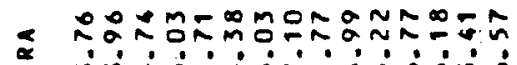

\& mंmidivioistiviviñ

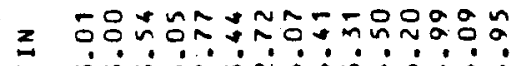

- minminiminisivinis:

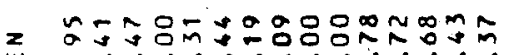

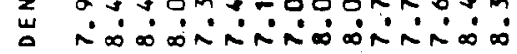

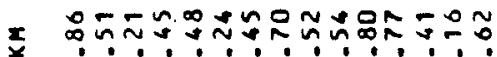

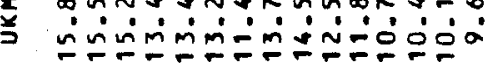

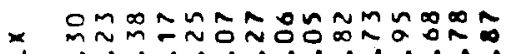

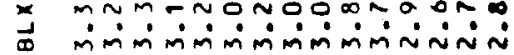

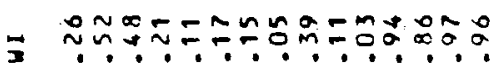

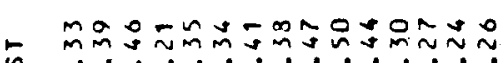

ค $\because \therefore \therefore \therefore \therefore \because \because \because$

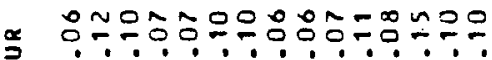

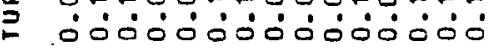

z

แ

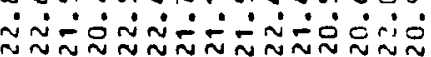

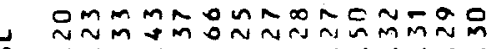

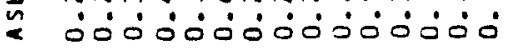

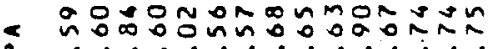

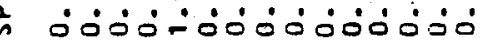

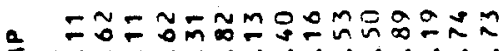

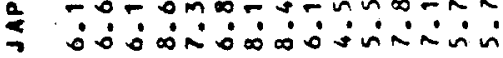

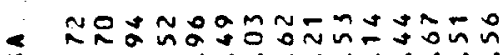

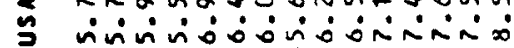

올

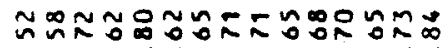

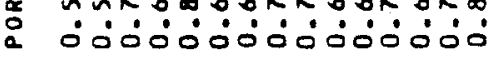

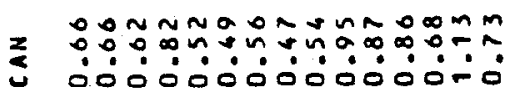

000000000000000

$\sum_{2}^{\stackrel{x}{2}}$

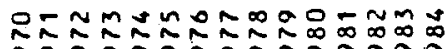
ấ

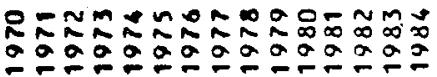




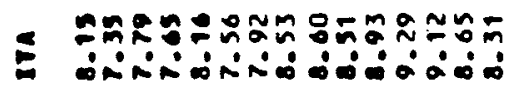

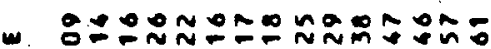

- dódódódódó0.

แ

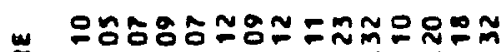

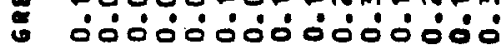

:

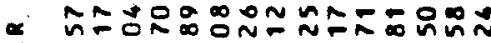

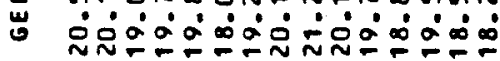

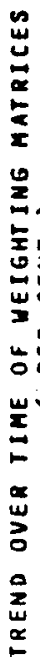

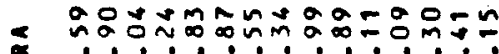

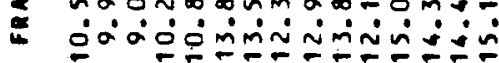

*

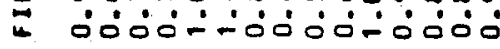

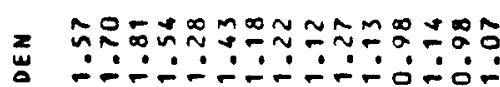

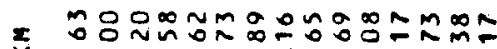

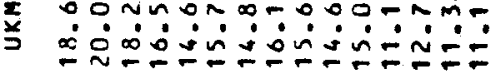

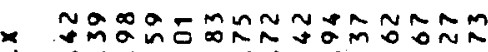

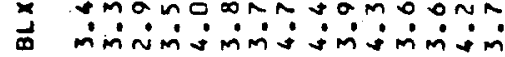

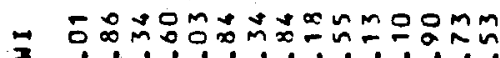

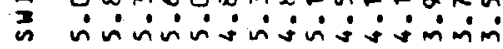

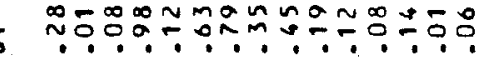

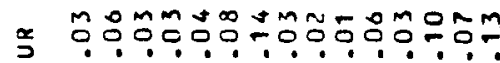

- ójodojódódó

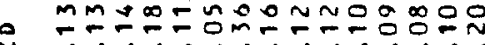

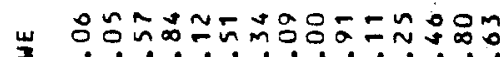

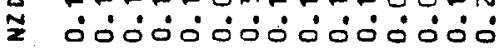

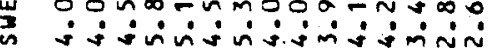

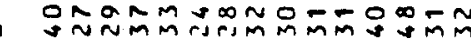

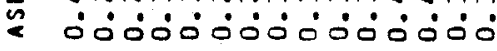

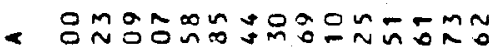

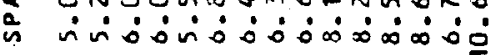

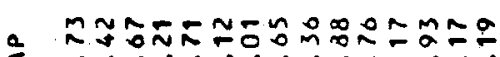

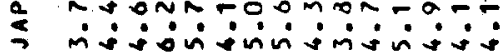

$\stackrel{0}{\circ}$

000000000000000

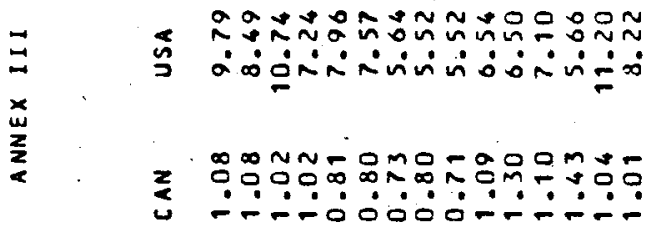

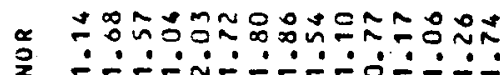

士

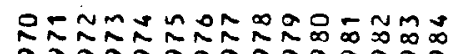

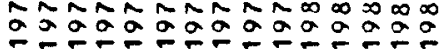

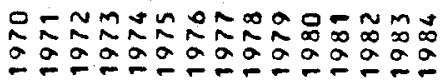




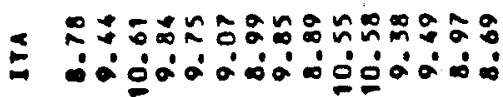

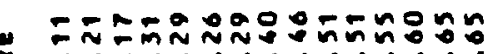

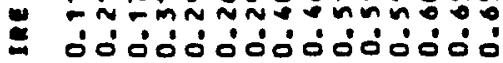

凹

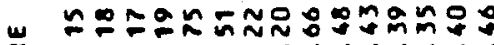

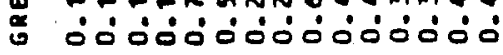

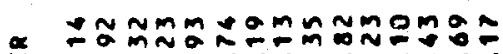

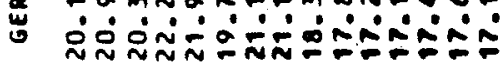

$a$

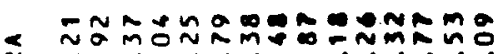

๕

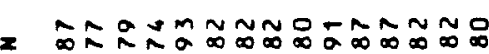

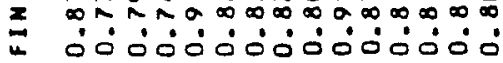

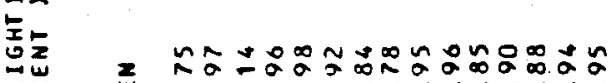

यू

$4 \stackrel{0}{*}$

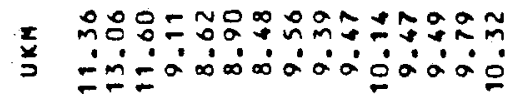

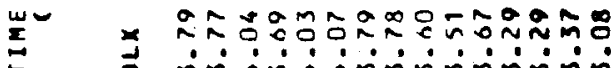

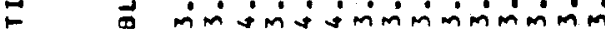

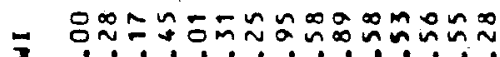

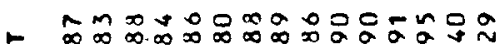

ڤ.

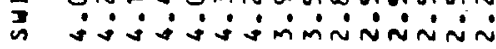

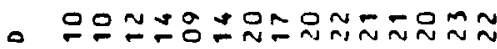

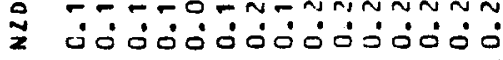

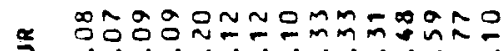

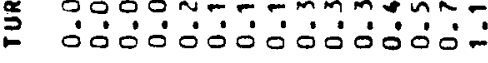

1 ํำ

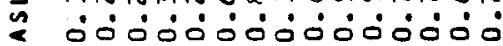

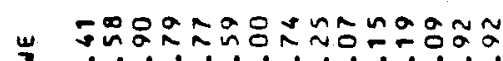

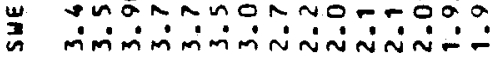

is

000000000000000

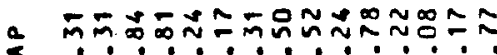

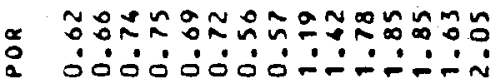

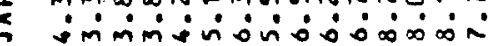

$\Xi \quad$ 药

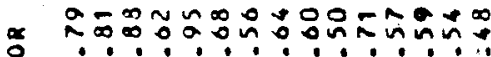

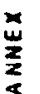

₹

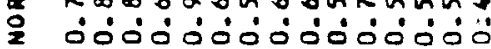

$=$

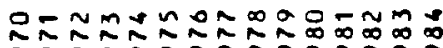

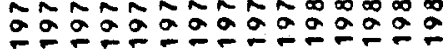




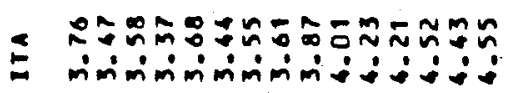

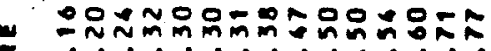

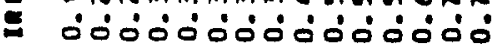

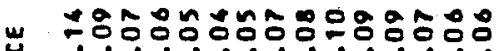

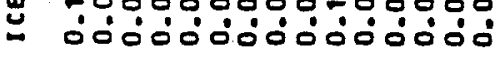

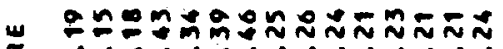

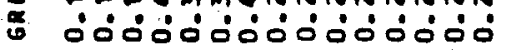

$\stackrel{m}{3}$

ะ

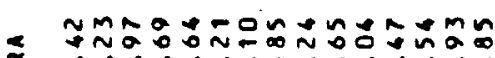

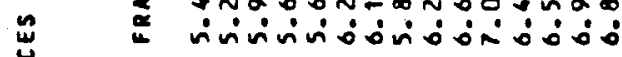

z

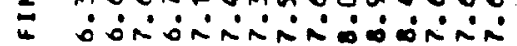

$z$ ำ

×

丂

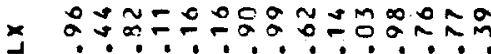

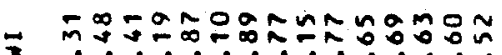

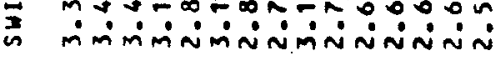

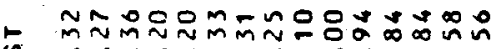

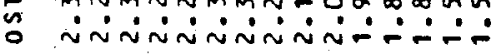

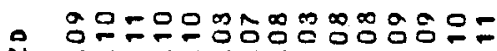

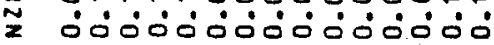

3

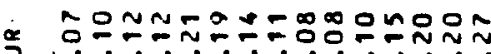

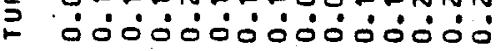

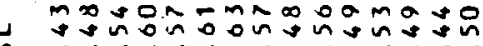

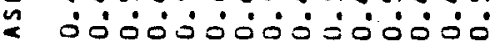

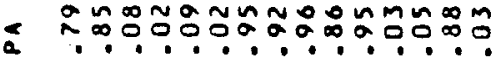

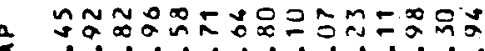

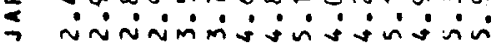

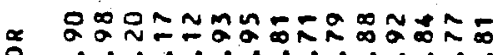

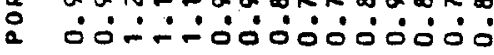

$\Xi$

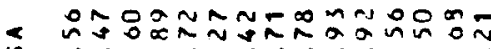

$\Rightarrow$ asiosisisinasis

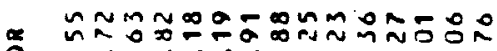

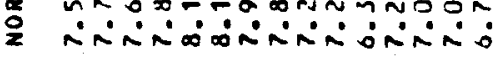

z.

z

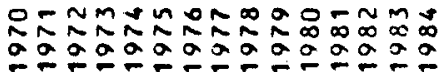

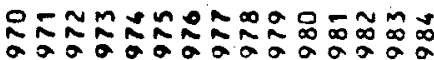




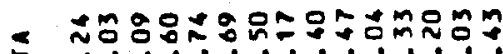

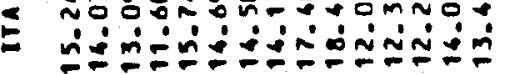

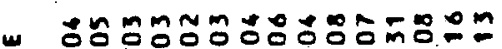

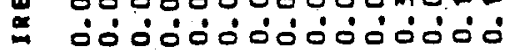

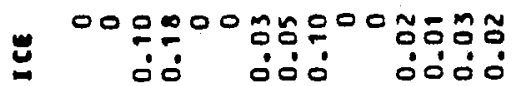

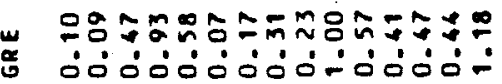

-

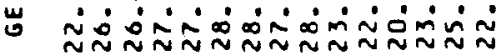

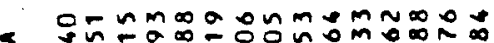

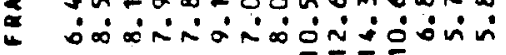

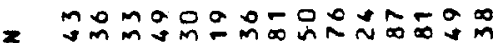

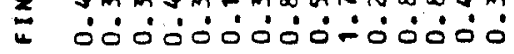

政

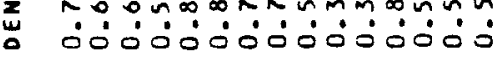

एᄂ

ब imjiminiminimimińm

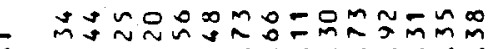

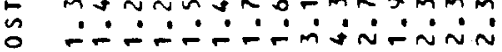

2

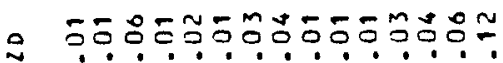

z

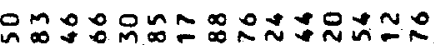

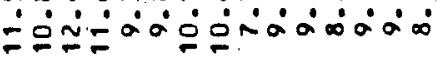

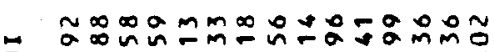

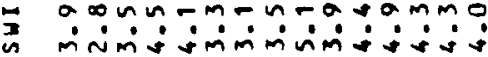

ก⿻上飞ัง

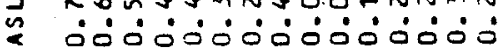

กำ:

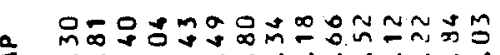

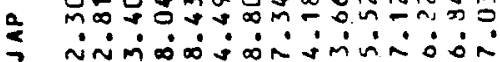

$\stackrel{\infty}{\circ}$

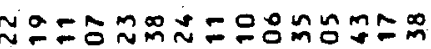

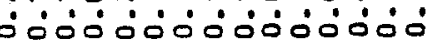

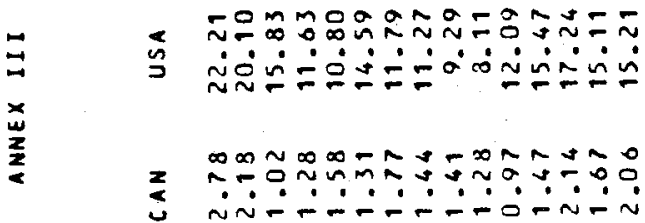

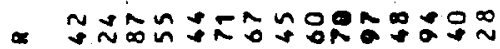

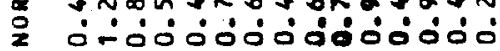

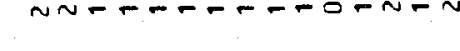

a

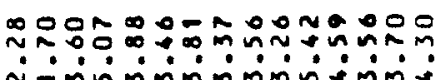




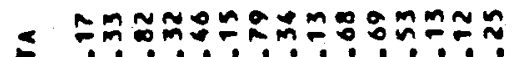

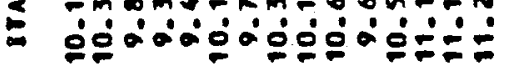

แ

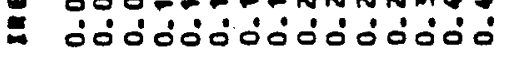

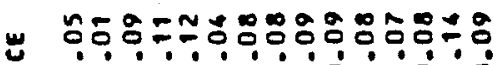

- dódojódódódó

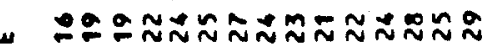

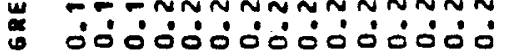

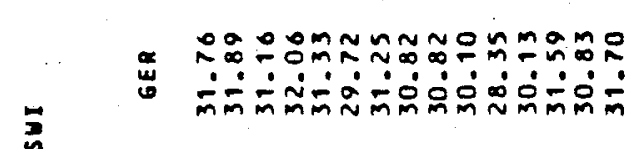

<

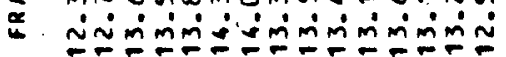

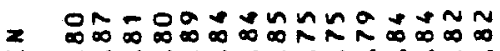

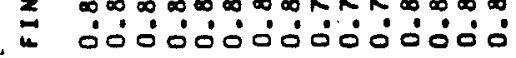

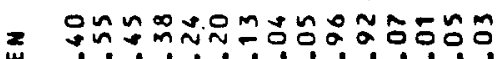

a

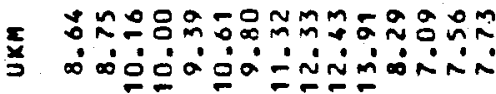

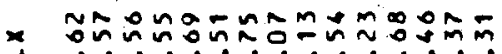

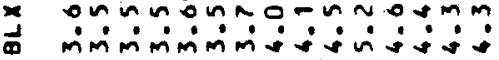

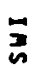

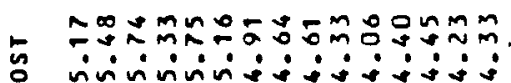

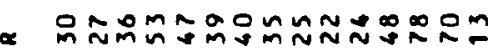

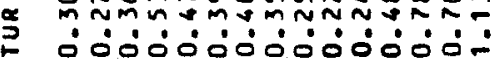

잉

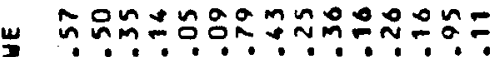

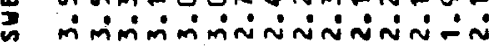

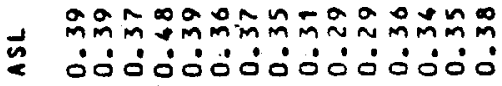

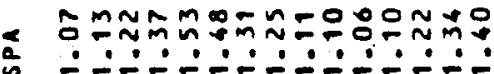

- 20ำ

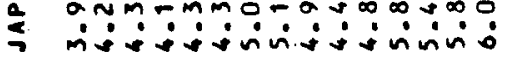

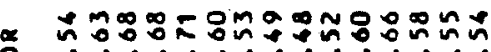

م

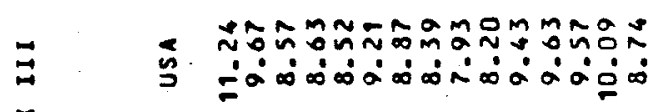

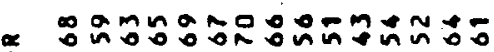

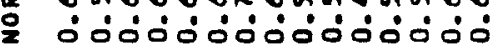

z

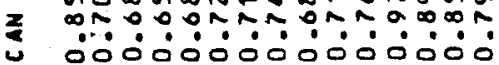

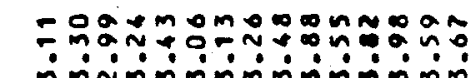

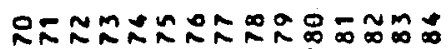

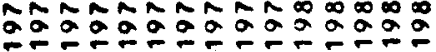

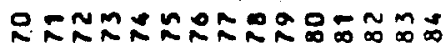

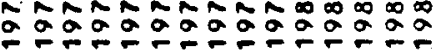




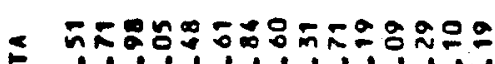

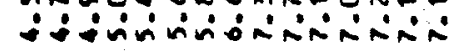

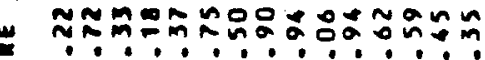

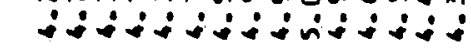

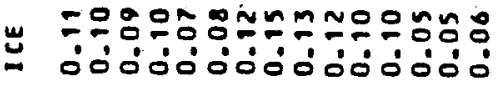

แ ํㅜำ品

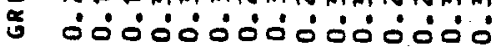

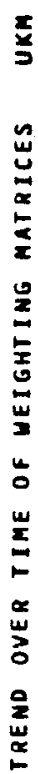

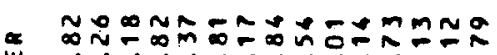

o

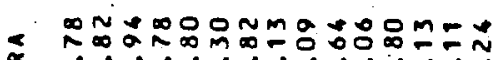

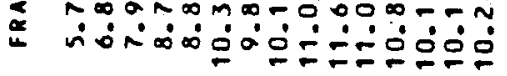

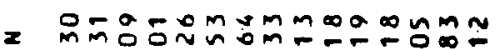

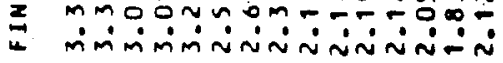

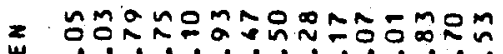

a ninitifimmimiñin

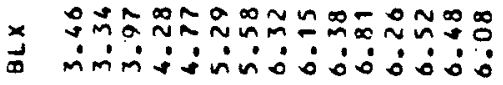

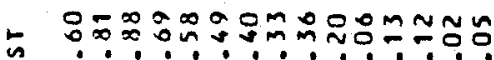

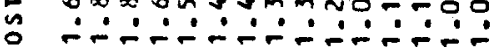

- Nล์

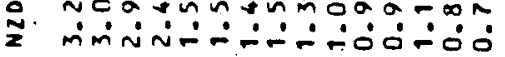

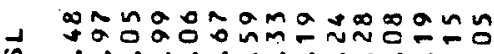

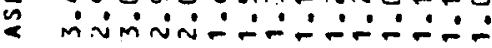

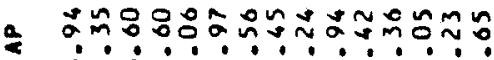

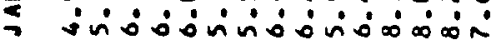

$=$
$=$
$w$
$z$
$z$
$z$

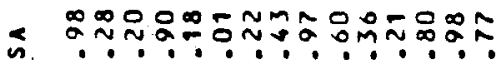

ร

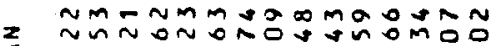

z

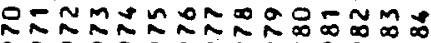

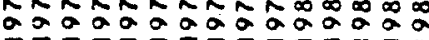

000000000000000

ב

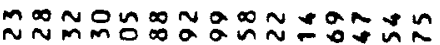

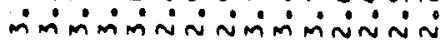

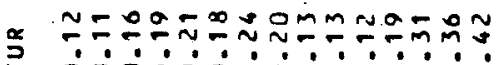

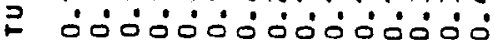

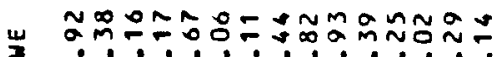

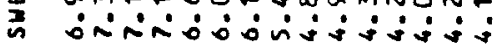

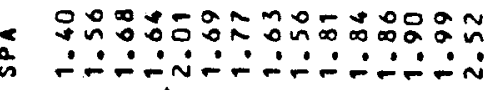

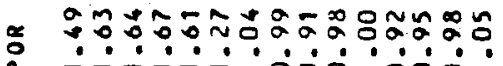

a -

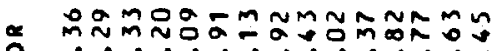

o miniminiminin:-1:

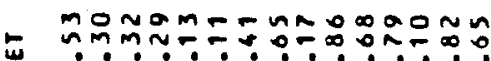

z

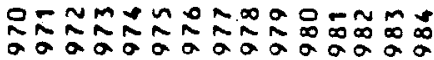


$-63-$

ANNEX 4 


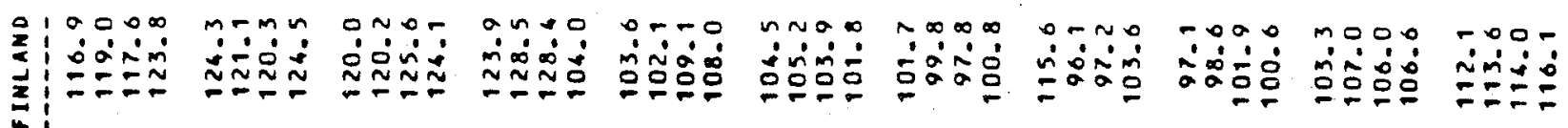

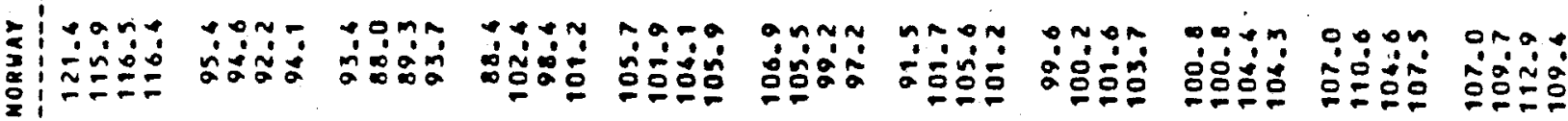

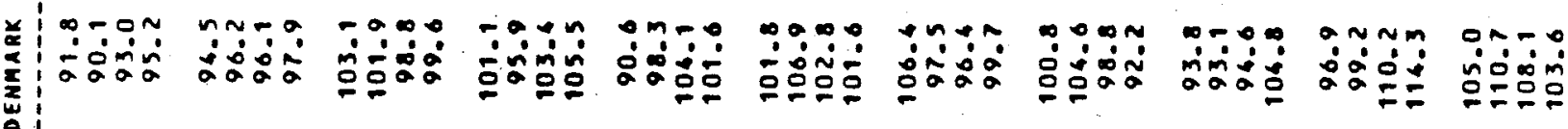

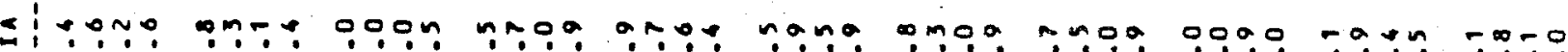
¿ 盗

1 nNND nON

$\simeq \cong \simeq \simeq$

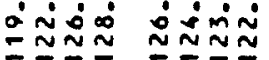

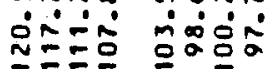

$+\infty$ no

ONO- nn

$a m: 0$

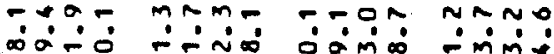

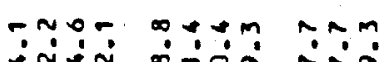

頮

in $0=n$ $+$

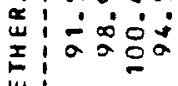

$+\infty \infty a$

:Oma -nMO ?nOm

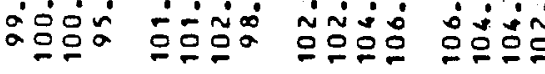

$\sim a \infty$

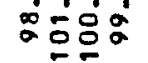

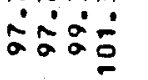

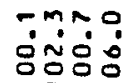

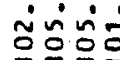

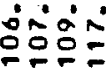

ร:?

$\because \div 0 \div$

?⿻ำ

$0+\infty \div$

$\because \because \infty \div$

:

$+\infty-\infty$

$\div a+0$

mo

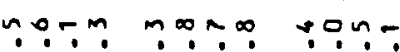

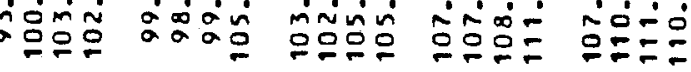

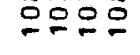

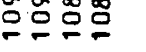

$\dot{0} \dot{0} \dot{0}=\dot{m}$

$\sim a ?$

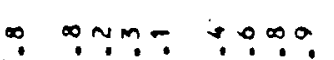

$p: 00$

on $0 m$

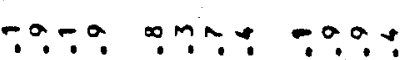

ว

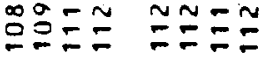

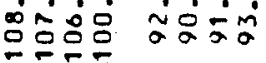

ñ⿻:

O̊ํํㅇ

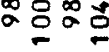

음으음

am? m?:?m.

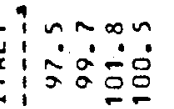

mo:?

$\infty \stackrel{\infty}{\infty} \sim \sim$

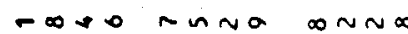

웅

$\because \simeq \infty$ in

oono ogjs nono

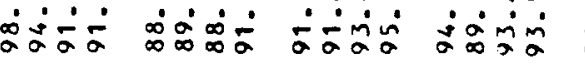

ins-

on-o

$\infty \pi n$

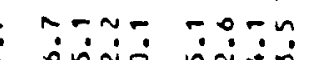

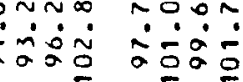

$0 \infty 00$

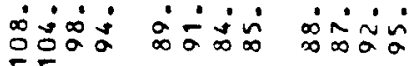

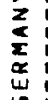

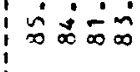

$\infty_{\infty} \infty \infty_{\infty}^{\infty}$

$\sin$

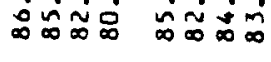

0000

$m u n a-00$

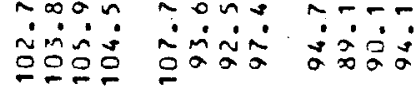

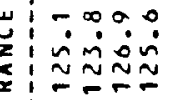

官品立

mom -n.т nomm

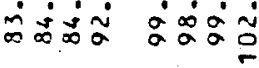

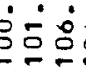

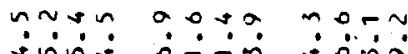

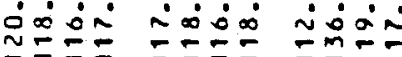

$\infty \div$

$\because-n$

$a 0 n$

음

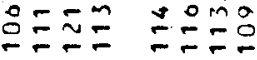

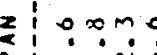

onon

Cand

Fニ̃

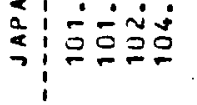

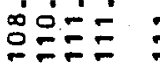

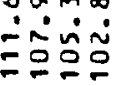

:

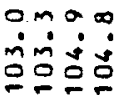

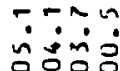

usna noaO

O $m \sim \infty$

onon amr-

-

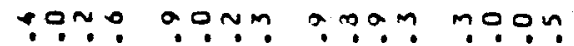

西

aro음

ํํㅇํํำ

$\because \dot{0}=\dot{\sim} \dot{\sim}$

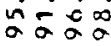

iो人응

má

$\cos 2$

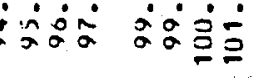

호ำ

num mmoo

non

$\sim \infty$

$\dot{m i j} \dot{j} \dot{i} \dot{0} \dot{0}$

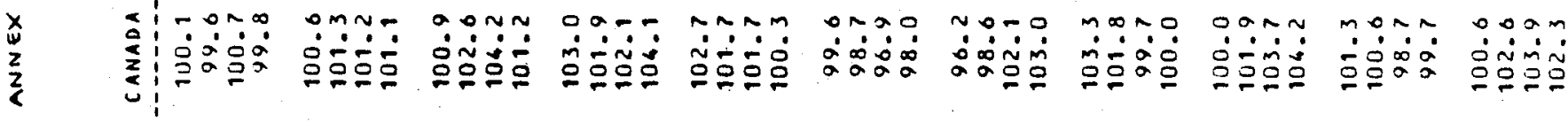

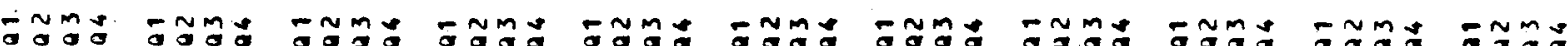

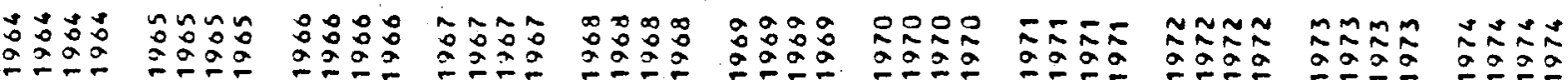




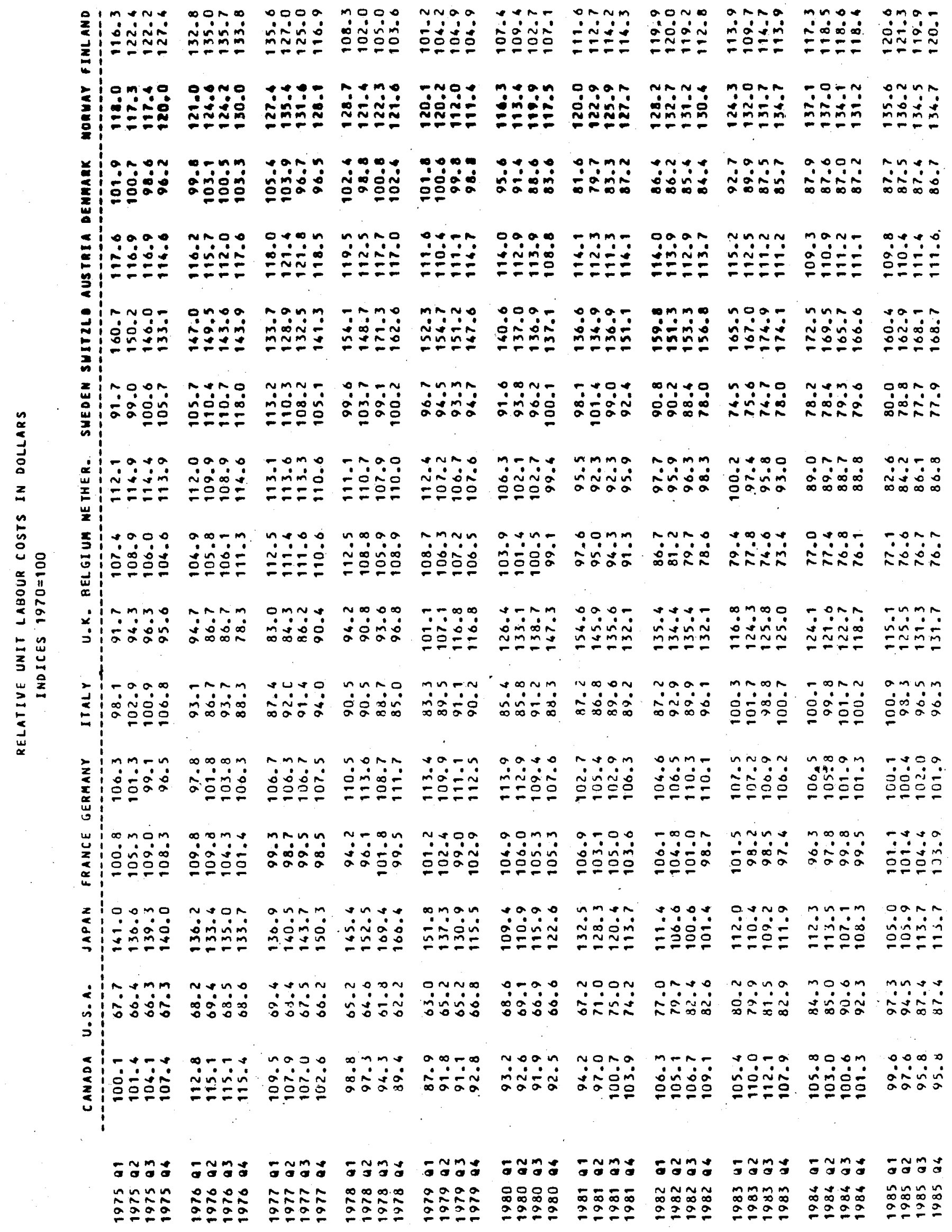




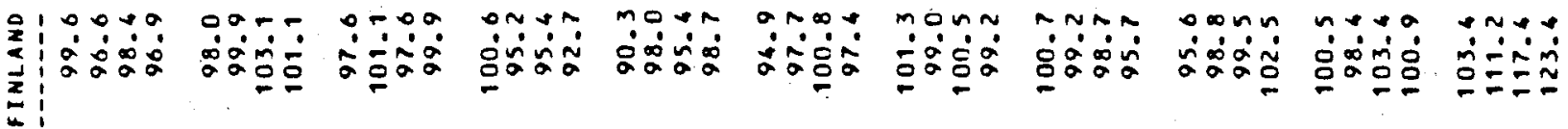

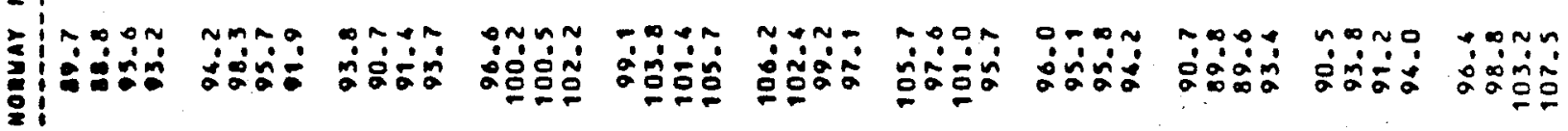

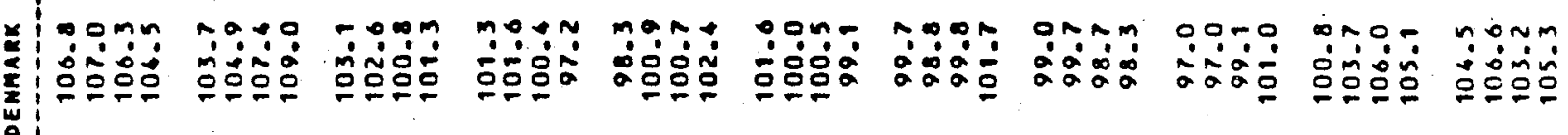

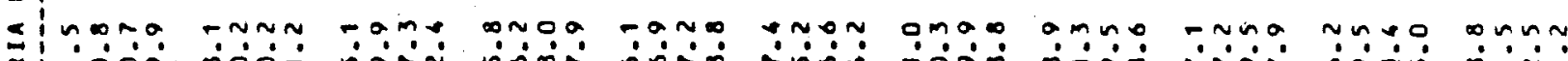

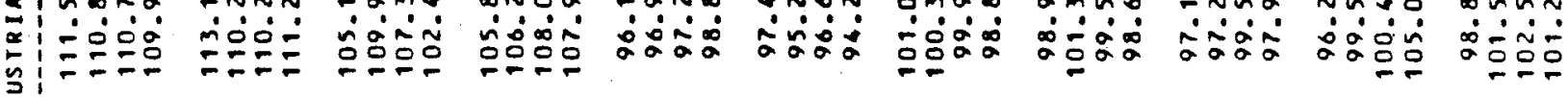
3

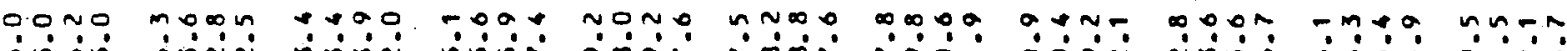

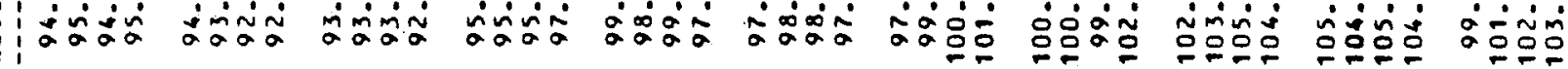

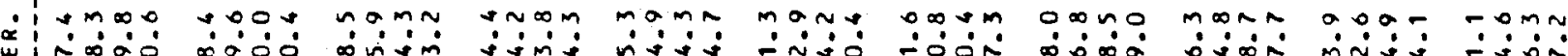
至 z

z:

nan gan? mon

$\infty$

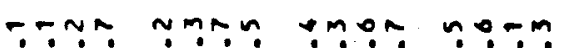

anno ompo nun

पिव்

㟔

$\ddot{\dot{y}}$

name nmon - nna

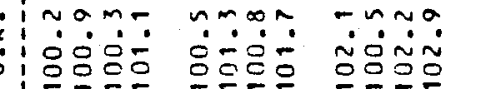

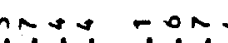

nน? m mono rn? : o

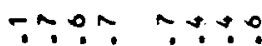
a

:

$\sin (x)$

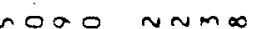

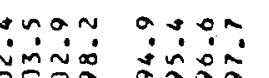

$0 \infty 00$

$000 m$ :0a0

ฮิ่ล์

aํํㅇ

ches

$\underset{z}{z}$

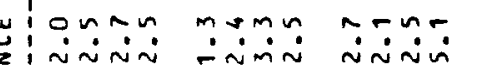

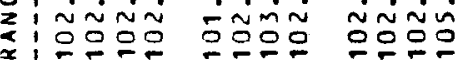

$\infty=0 n m 0-\infty$

inno

흐므응

कूँवें

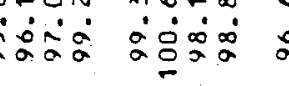

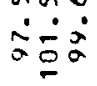

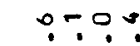

응융ㅁㅇ

inmo

$-n-a$ opon

บㄱ. ㄱ. ㄱ. noom

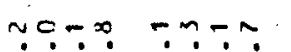

$\ddot{m} \dot{0} \div \dot{0}$

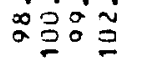

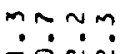

(a)

$\therefore \sim a 0$

gnmm vaon

$\geq$

anno muno nNon

:

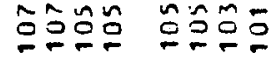

으을응

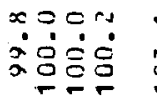

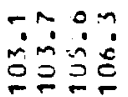

$m x+n$

$n \rightarrow n n$ ovo0

$m .90 .900$

midn

$\because \because a$

$\therefore$

$\infty 0 \mathrm{~m}-$

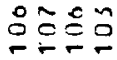

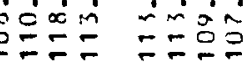

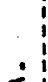

si

约

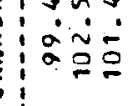

-nme -nme -nma gnma

$\because \because \div$

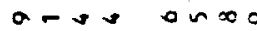

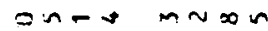

\section{$\dot{2}$}

$m n m$ Ya-

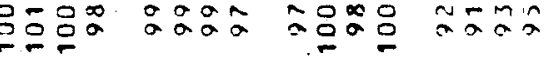

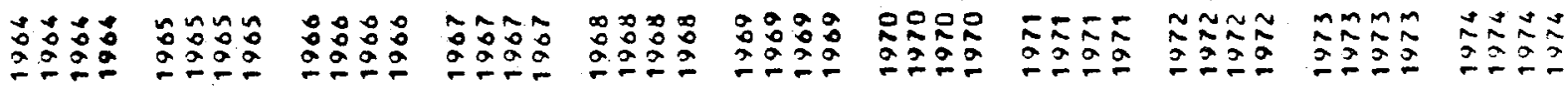




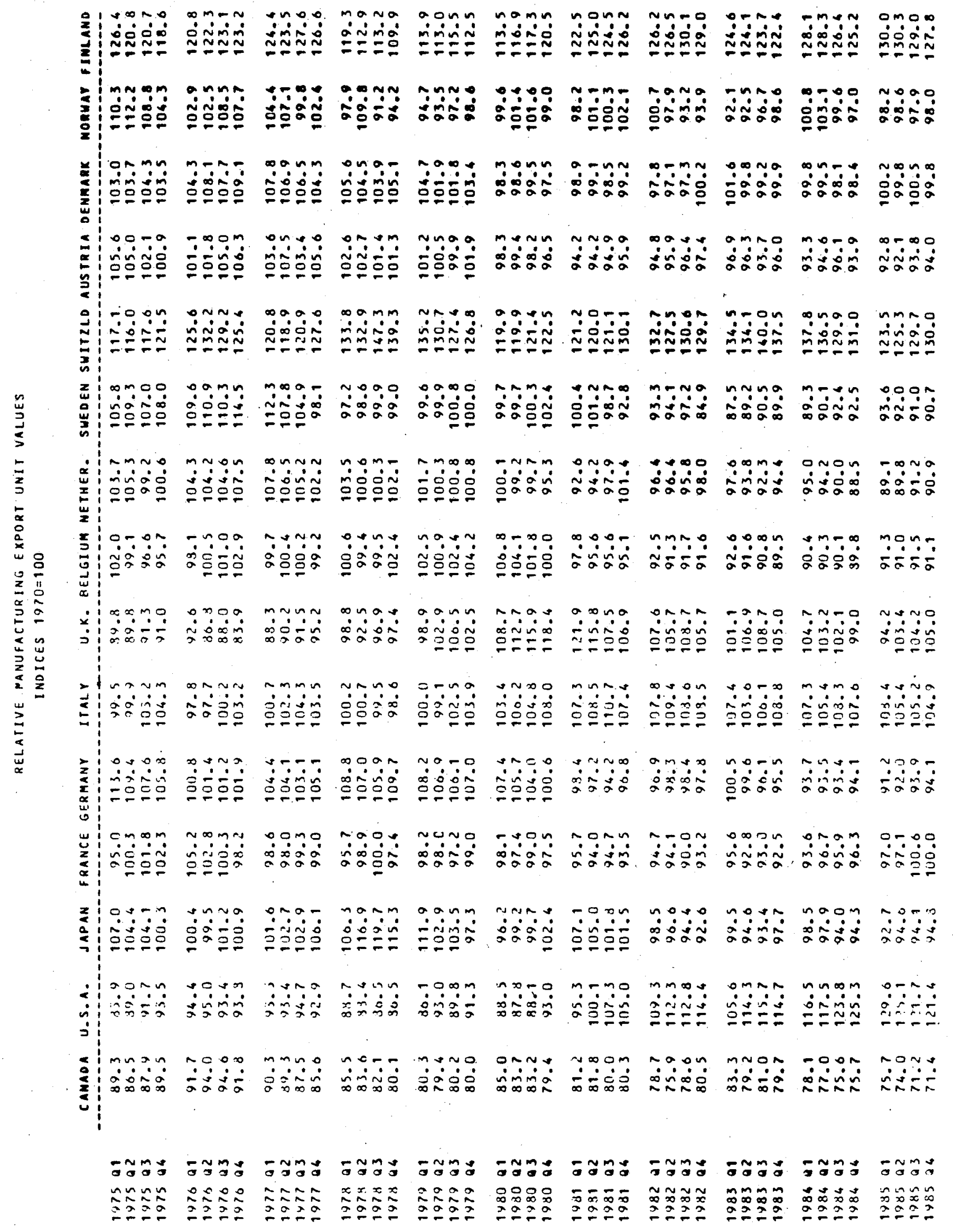




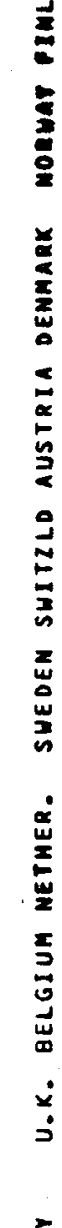

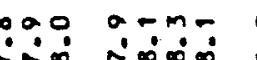

: $\infty$

hoos oun

$\cos 20$ 西:

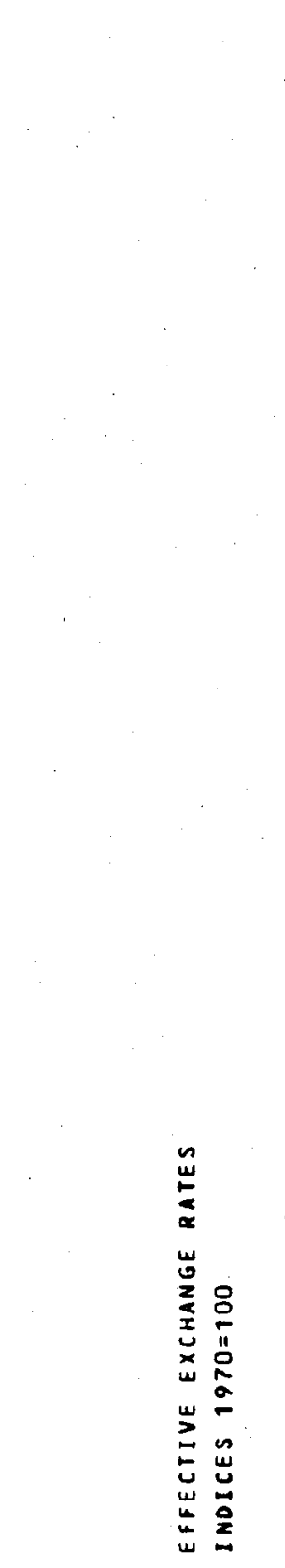

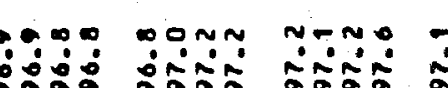

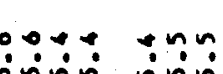

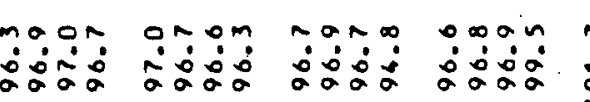

\section{ข}

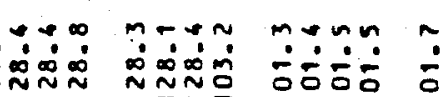

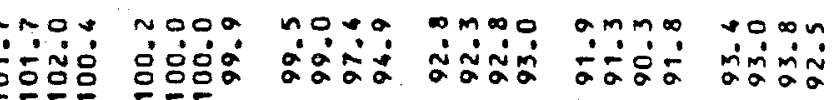

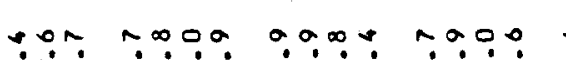

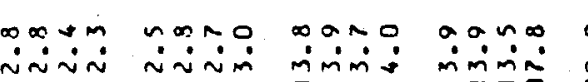

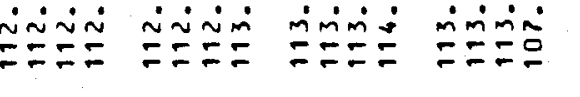

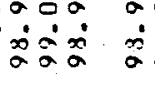

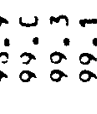

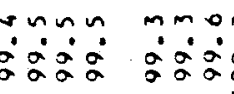

iin

\section{ณiv}

\section{colo}

\section{:}

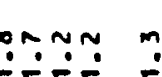
$m-m \sim$ ํ. mon mone nom

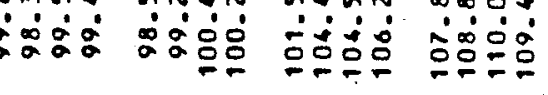

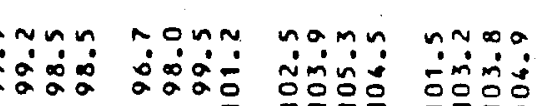

\section{-ON?

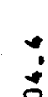

\section{$m m ? n$}

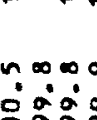

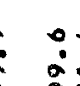

*or mmoa norn vno 等

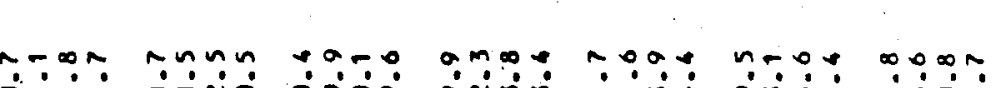

ำ

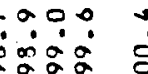

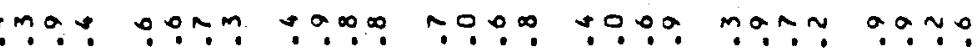

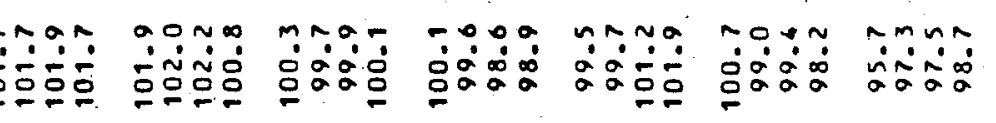

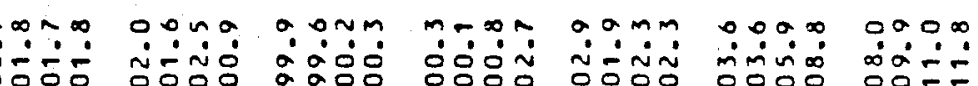

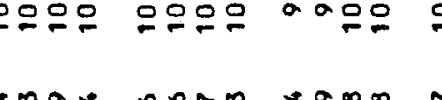

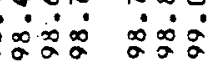

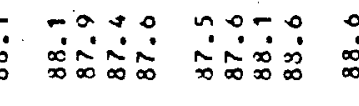

(n):<smiles>C=C</smiles>

(1) 


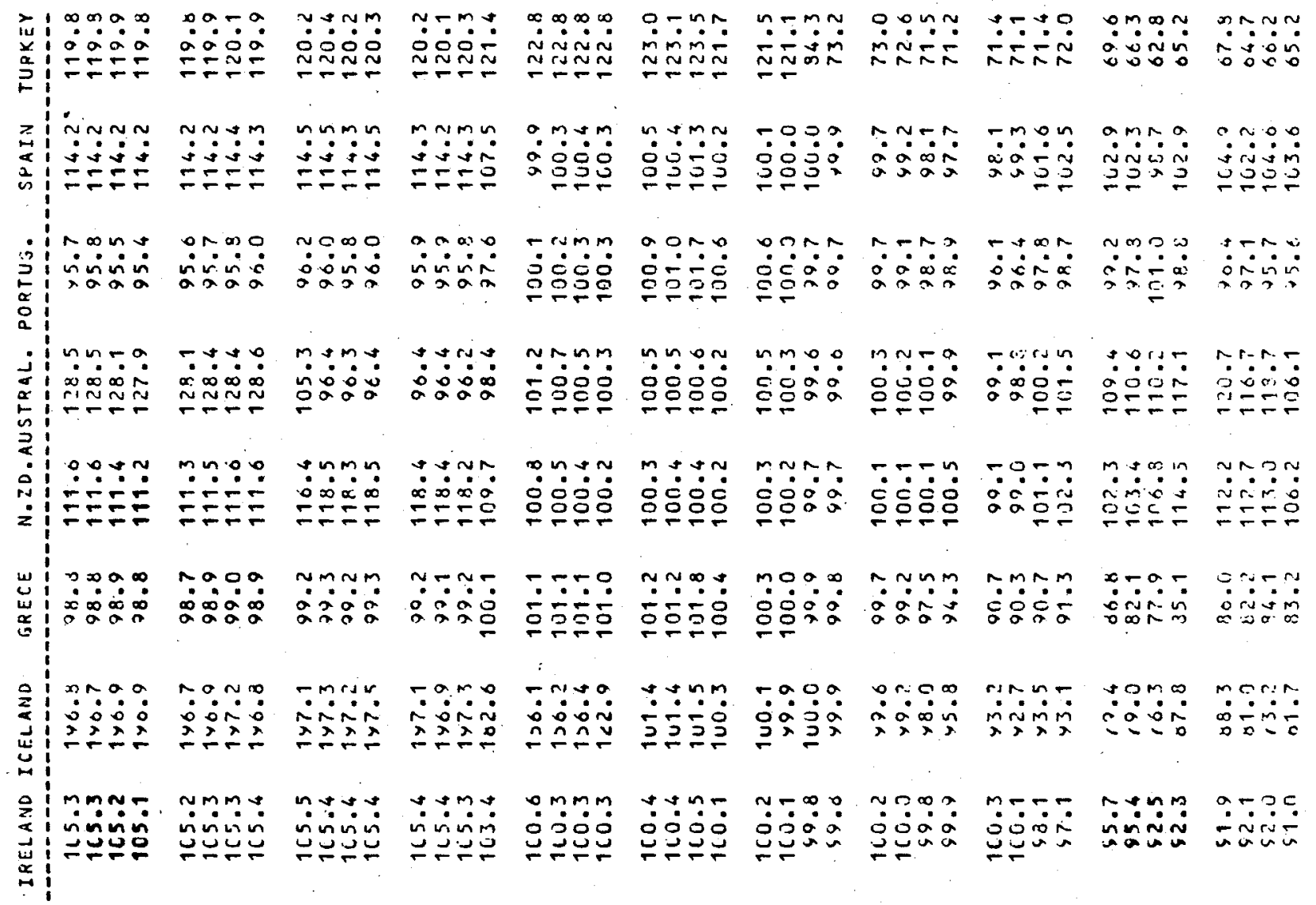

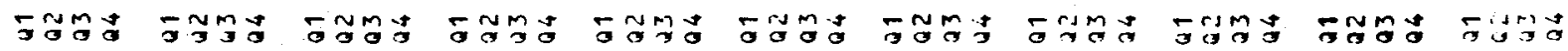

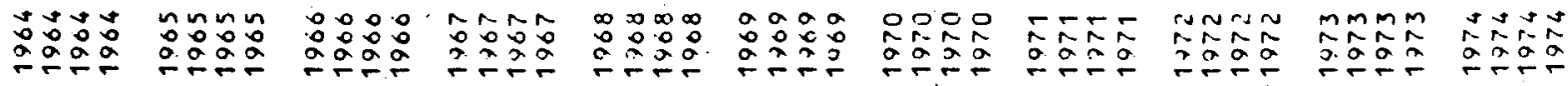




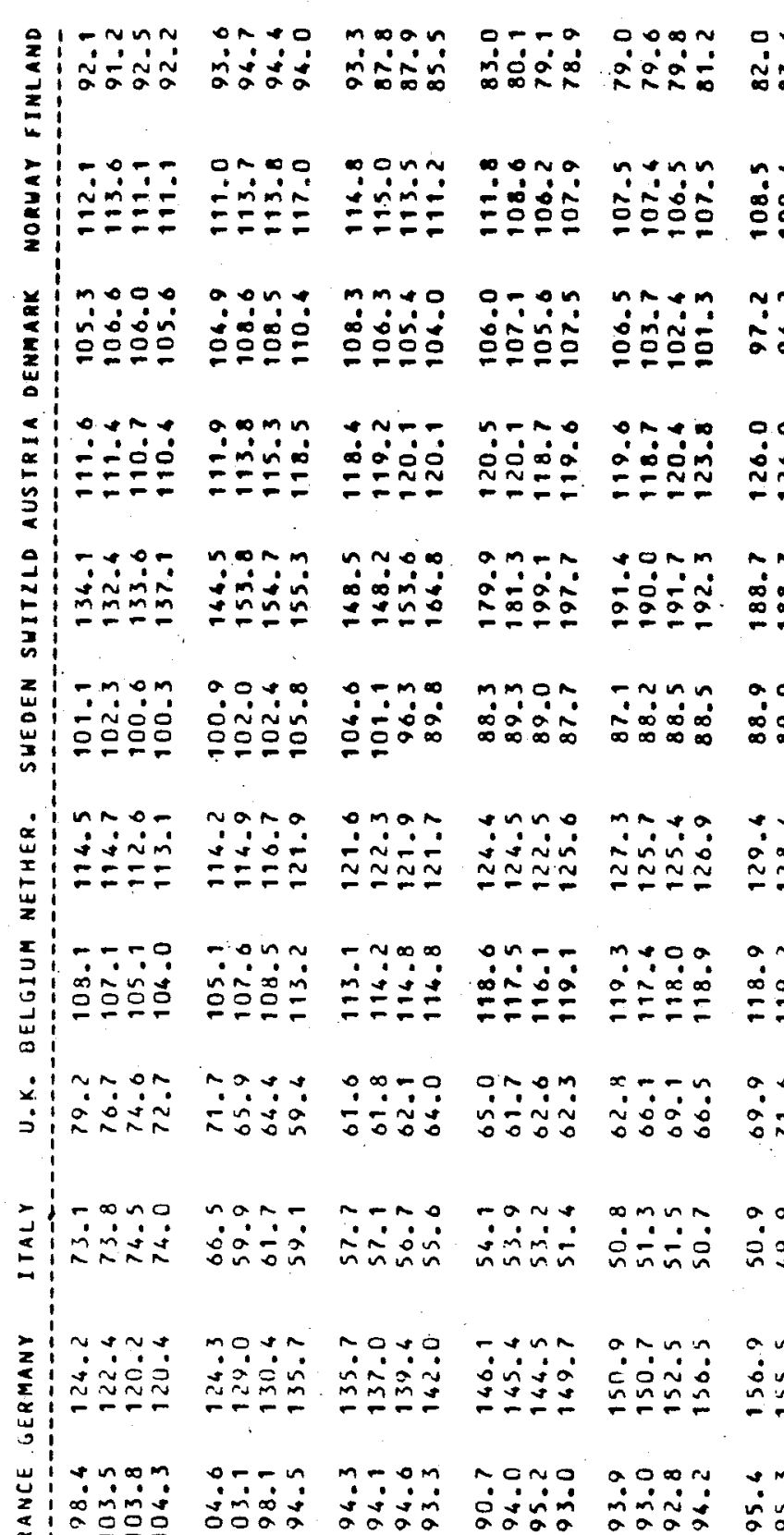

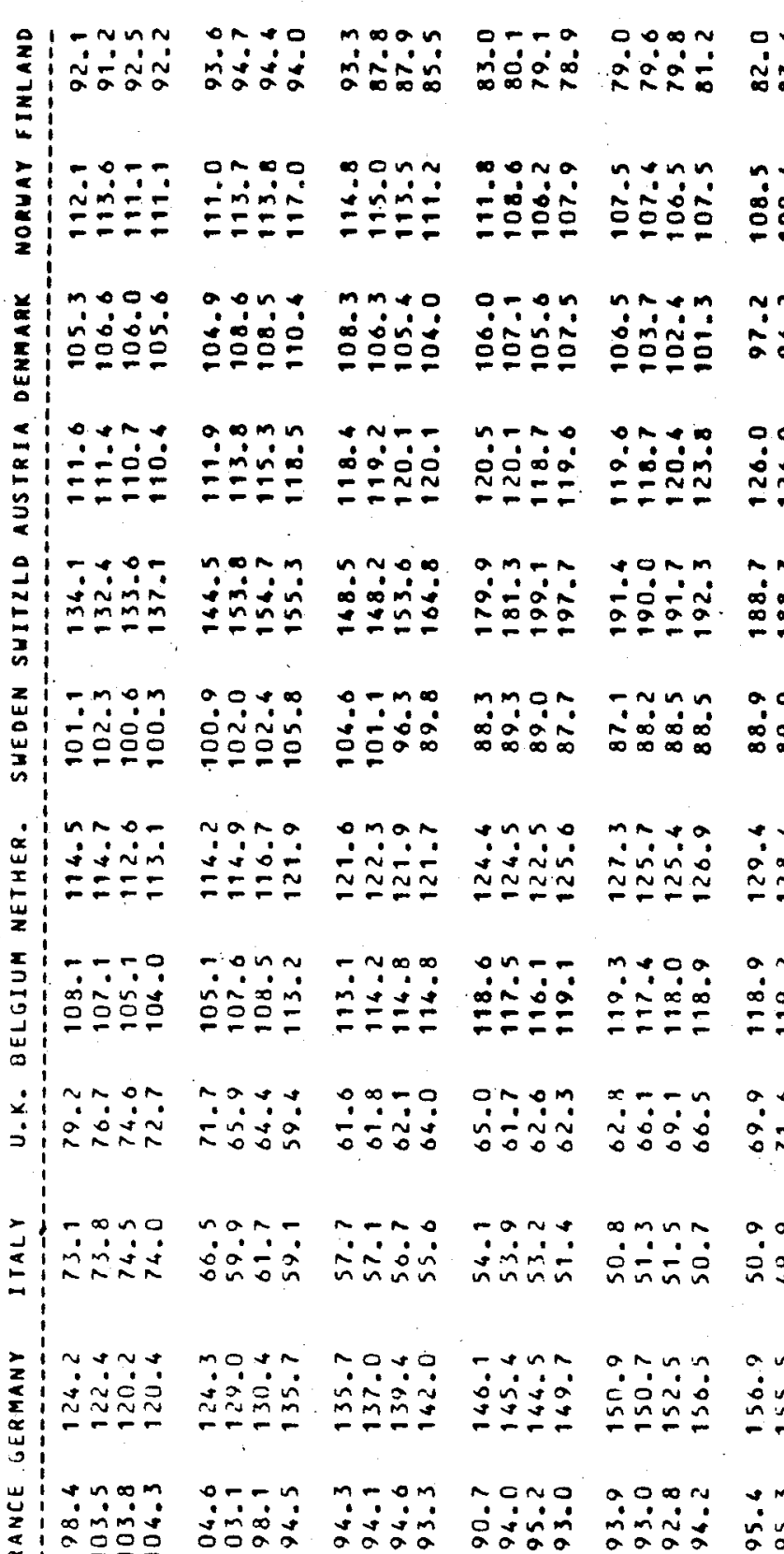

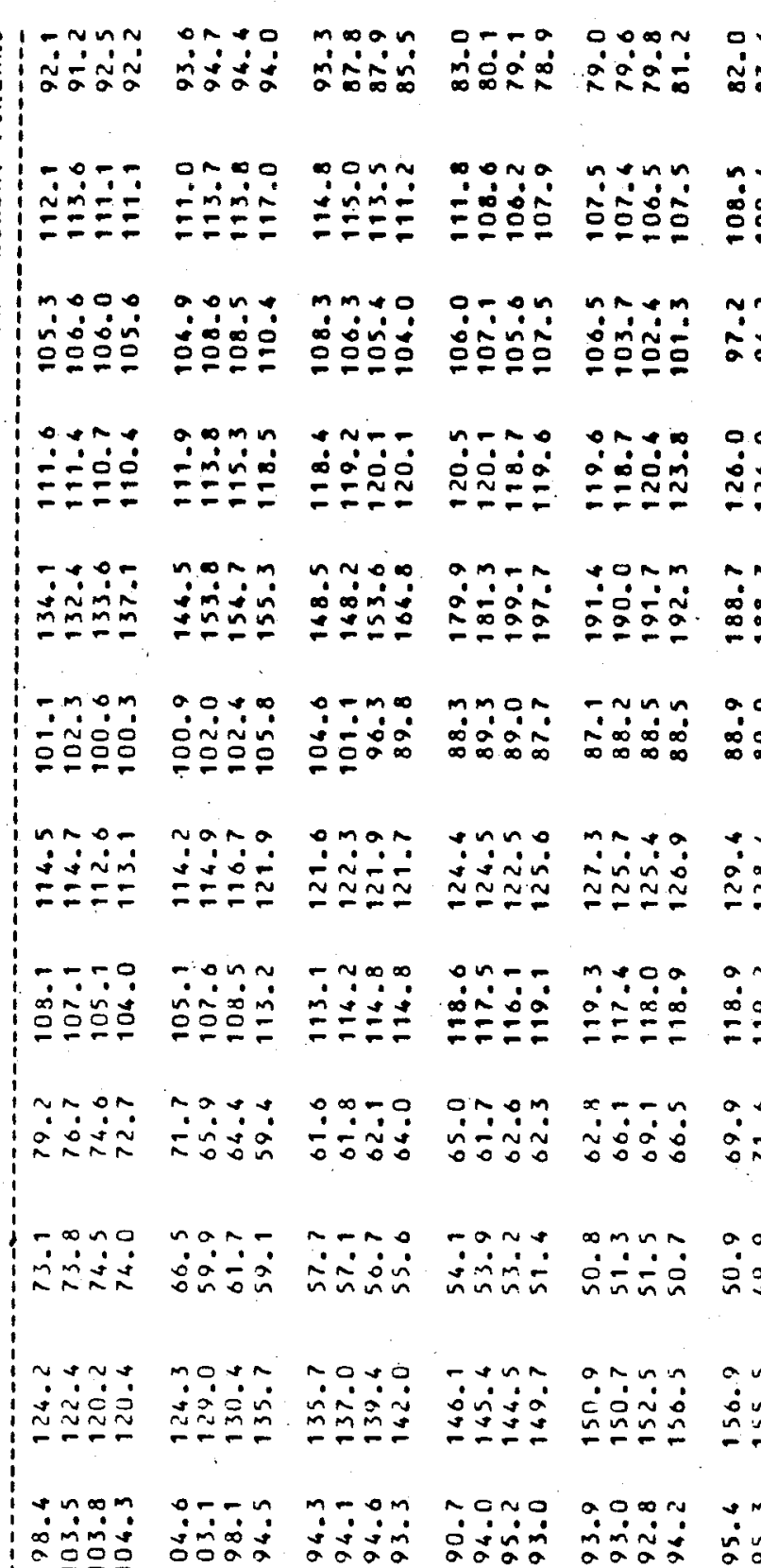

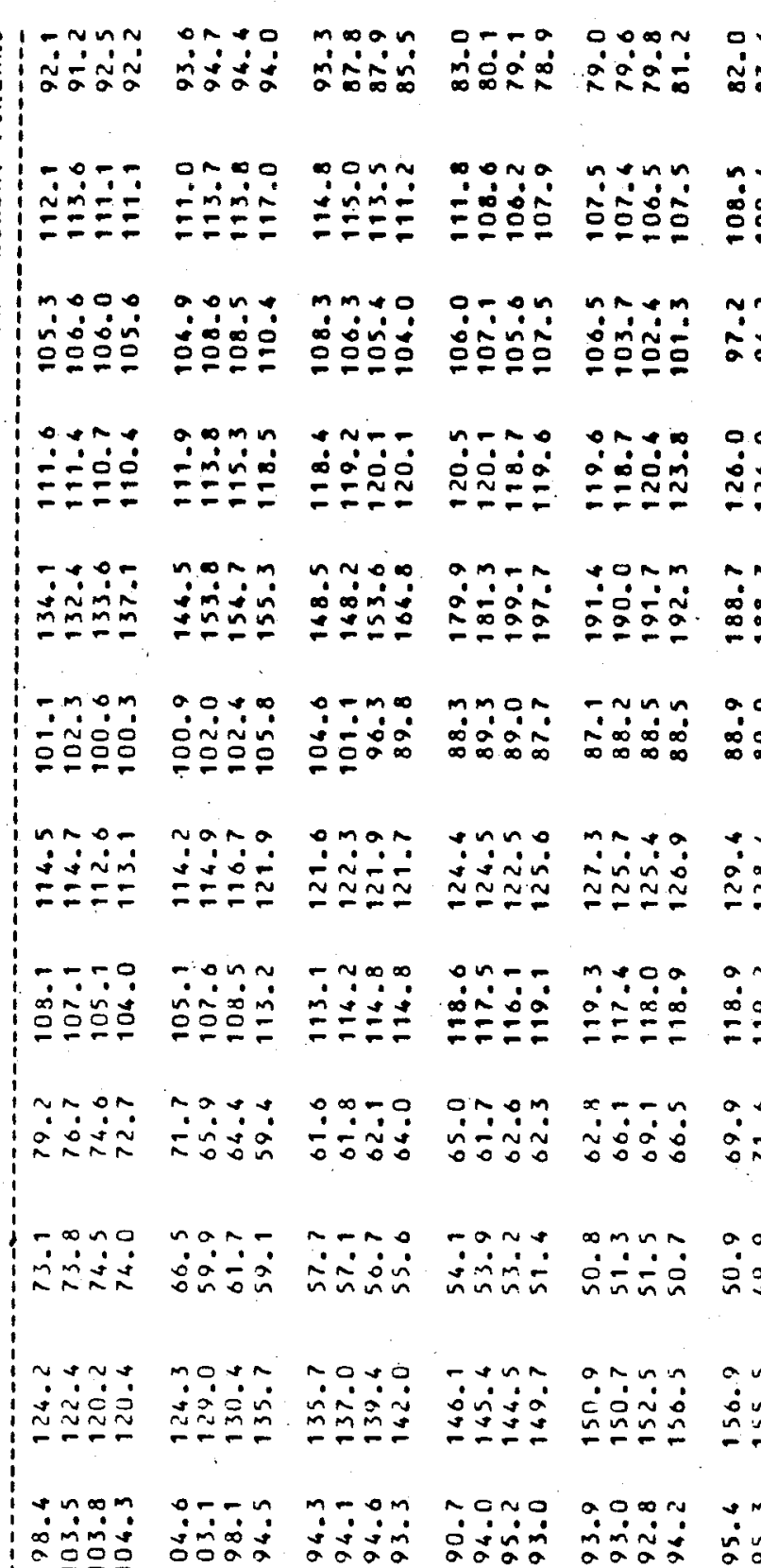

:

\section{:} m

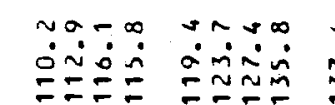

(1)

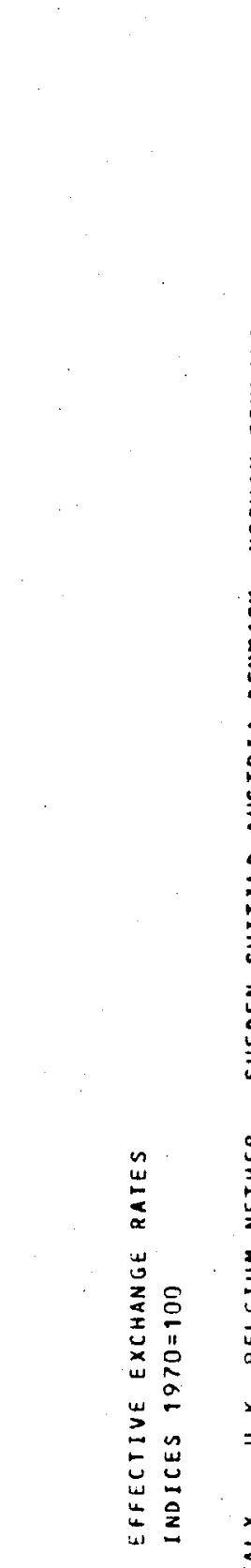




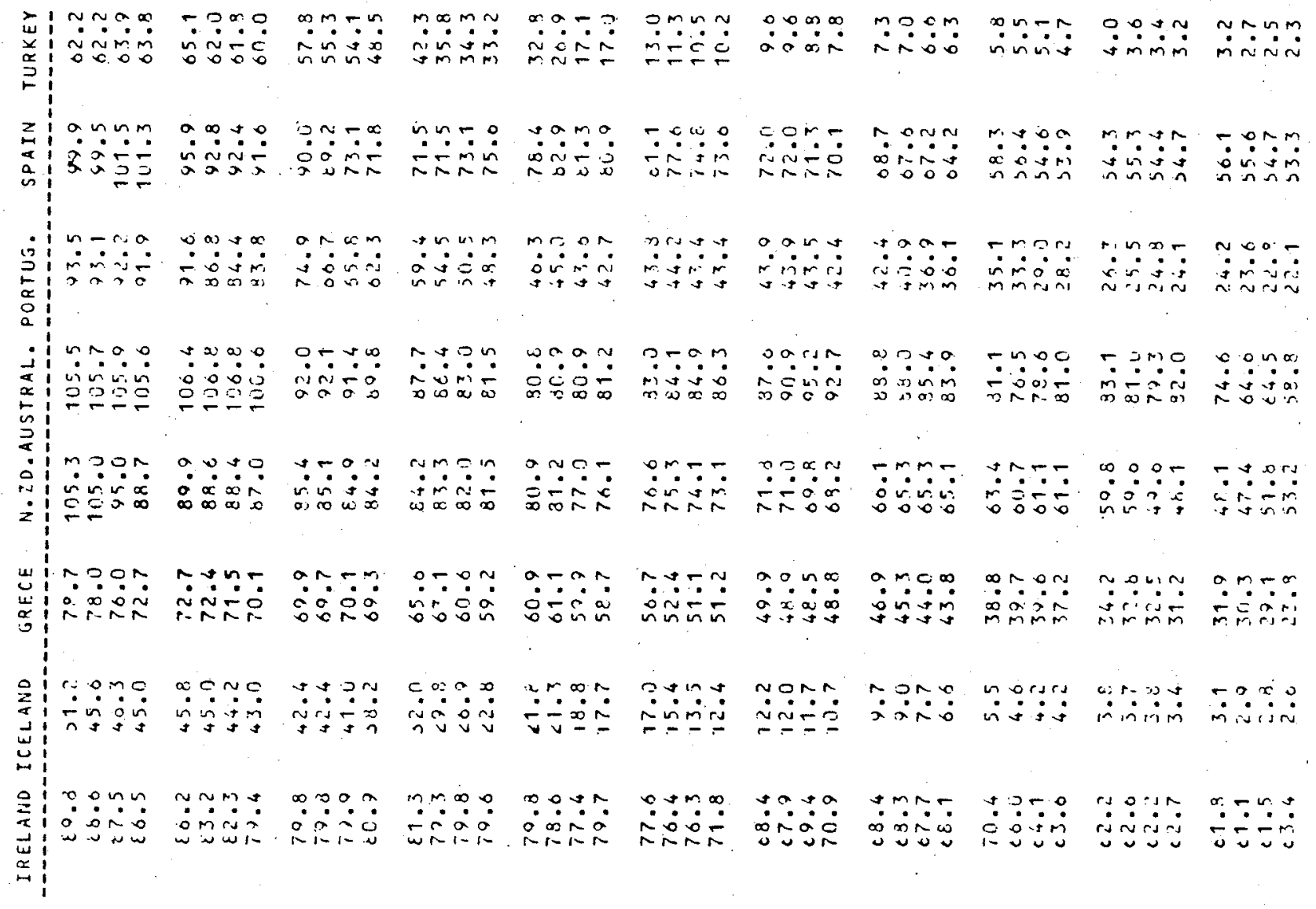

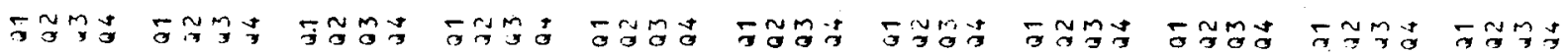

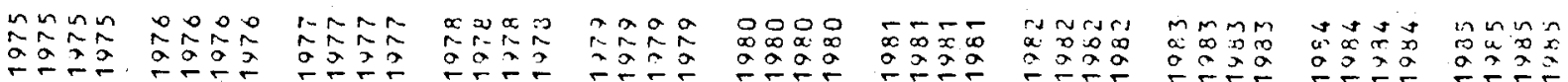


